\title{
Critically Evaluated Energy Levels and Spectral Lines of Singly Ionized Indium (In II)
}

\author{
A. Kramida \\ National Institute of Standards and Technology, \\ Gaithersburg, MD 20899 \\ alexander.kramida@nist.gov
}

\begin{abstract}
A comprehensive list of the best measured wavelengths in the In II spectrum has been compiled. Uncertainties of the wavelength measurements have been analyzed, and existing inconsistencies have been resolved. An optimized set of fine-structure energy levels that fits all observed wavelengths has been derived. Uncertainties of the energy level values have been reduced by an order of magnitude. An improved value of the ionization limit of In II has been determined by fitting quantum-defect and polarization formulas for several series of levels. Intensities of lines observed by different authors have been analyzed and converted to a uniform scale. A set of recommended values of radiative transition rates has been critically compiled, and uncertainties of these rates have been estimated. The hyperfine structure interval in the $5 \mathrm{~s}{ }^{2} \mathrm{~S}$ ground state of In III has been determined from the measurements of the $5 \mathrm{~s} n \mathrm{~g}$ and $5 \mathrm{~s} n \mathrm{~h}$ series in In II.
\end{abstract}

Key words:: atomic energy levels; hyperfine structure; ionization potentials; singly ionized indium; spectral lines; transition probabilities.

Accepted: December 18, 2012

Published: January 14, 2013

http://dx.doi.org/10.6028/jres.118.004

\section{Introduction}

Singly ionized indium, isoelectronic with $\mathrm{Cd} \mathrm{I}$, has a ground state $[\mathrm{Kr}] 4 \mathrm{~d}^{10} 5 \mathrm{~s}^{2} \mathrm{~S}_{0}$. Its photon emission spectrum has been investigated by a number of researchers since the beginning of the $20^{\text {th }}$ century (see the current bibliography on this spectrum in the Atomic Energy Levels and Spectra Bibliographic Database [1] of the National Institute of Standards and Technology (NIST)). The most important of the early contributions is the work of Paschen and Campbell [2]. They presented a list of about 500 spectral lines of In II, 325 of which had partially resolved and analyzed hyperfine structure (hfs) patterns. From those observed spectral lines they derived a list of 180 fine-structure energy levels and derived the first ionization limit at $152195 \mathrm{~cm}^{-1}$. Paschen and Campbell observed the spectrum from $2078 \AA$ to $9246 \AA$, but adopted a value of $43349 \mathrm{~cm}^{-1}$ for the $5 \mathrm{~s}^{2}{ }^{1} \mathrm{~S}_{0}-5 \mathrm{~s} 5 \mathrm{p}{ }^{3} \mathrm{P}^{\circ}$ separation, as determined earlier by Lang and Sawyer [3] whose observations covered a wider range from $680 \AA$ to $7277 \AA$. The energy levels given in the Atomic Energy Levels compilation (AEL) by Moore [4] were quoted from Paschen and Campbell [1]. The spectrum from $680 \AA$ to $5122 \AA$ was re-observed in 1969 by Bhatia [5] who identified many transitions previously unknown. Sansonetti and Martin [6] re-analyzed the fine-structure data of Paschen and Campbell together with Bhatia's observations and found that the positions of all excited levels above and including the $5 \mathrm{~s} 6 \mathrm{~s}^{3} \mathrm{~S}_{1}$ level, as well as the ionization limit, have to be shifted upwards by $4.37(30) \mathrm{cm}^{-1}$ from the values given by Paschen and Campbell.

Interest in the In II spectrum greatly increased in the early 1980s, when it was realized that the strongly forbidden $5 \mathrm{~s}^{2}{ }^{1} \mathrm{~S}_{0}-5 \mathrm{~s} 5 \mathrm{p}^{3} \mathrm{P}_{0}^{\circ}$ transition can be exploited in single-ion traps to construct a high-precision atomic clock [7]. This transition, strictly forbidden in unperturbed atomic spectra, is induced by the 
hyperfine interaction. With the clock application in mind, Larkins and Hannaford [8] have measured the absolute frequencies of hyperfine components of several transitions in ${ }^{115} \mathrm{In}^{+}$, from which they derived the corresponding energy levels and hfs interaction constants. In particular, the wave number of the abovementioned clock transition was found to be $42275.986(7) \mathrm{cm}^{-1}$. This result was confirmed two years later by Peik et al. [9]. In 2001, von Zanthier et al. [10] refined this wave number by many orders of magnitude. Their result, $42275.995245348(8) \mathrm{cm}^{-1}$, agrees with both previous measurements.

In the same year 2001, Karlsson and Litzén [11] accurately measured wavelengths of 53 In II lines using a Fourier transform spectrometer. From these measurements, they derived values for 38 energy levels of In II, much improved compared to Moore's AEL [4]. For the $5 \mathrm{~s} 5 \mathrm{p}{ }^{3} \mathrm{P}_{0}^{\circ}$ level, Karlsson and Litzén obtained $42275.997 \mathrm{~cm}^{-1}$. They did not specify the uncertainty of this value, but mentioned that all their energy level values have uncertainties ranging from $\pm 0.001 \mathrm{~cm}^{-1}$ to $\pm 0.020 \mathrm{~cm}^{-1}$. Their result for the forbidden $5 \mathrm{~s}^{2} \mathrm{~S}_{0}-5 \mathrm{~s} 5 \mathrm{p}^{3} \mathrm{P}_{0}^{\circ}$ transition is in good agreement with all previously mentioned data. The agreement is not so good for other transitions. In particular, for the four other transitions measured by Larkins and Hannaford [8], the wave numbers measured by Karlsson and Litzén disagree by amounts far exceeding the combined measurement uncertainties. For the energy levels above $90000 \mathrm{~cm}^{-1}$, Karlsson and Litzén arrived at conclusions similar to those of Sansonetti and Martin [6]. Namely, they concluded that all these levels and the ionization limit determined by Paschen and Campbell [2] should be shifted upwards by $5.16 \mathrm{~cm}^{-1}$. This shift value is greater than that determined by Sansonetti and Martin by $0.79 \mathrm{~cm}^{-1}$. Since the lines observed by Karlsson and Litzén represent a small subset of all lines observed by Paschen and Campbell and by Bhatia, this indicates that the level shifts are not the same for all levels. The individual level shifts should be determined by re-analyzing all observed data accounting for measurement uncertainties. It was noted by Smirnov [12] that, by combining the highly accurate levels values from Karlsson and Litzén [11] with wavenumbers measured by Paschen and Campbell [2], it is possible to determine many of the levels not included in [11] with uncertainties as small as $\pm 0.01 \mathrm{~cm}^{-1}$.

Karlsson and Litzén [11] pointed out that the levels $5 \mathrm{~s} 5 \mathrm{~d}^{1} \mathrm{D}_{2}$ and $5 \mathrm{p}^{21} \mathrm{D}_{2}$ are strongly mixed, and their designations as given in Moore's AEL [4] should be reversed. This revision was not reflected in Sansonetti and Martin [6].

Another problem not addressed previously is related to relative intensities of observed lines. In the selection of In II lines included in the NIST publications [13,14], the line intensities were determined by summing up the intensities of the hyperfine components, as given by Paschen and Campbell [2]. However, the latter authors used several different spectrographs, some with prisms and some with gratings, and, in the case of gratings, observed lines in different orders of diffraction. Intensities of lines registered by different methods were actually on different scales. According to my analysis, the sensitivity of different registration methods used by Paschen and Campbell varies greatly (often by orders of magnitude), and the corresponding intensity scales have different wavelength dependences. In addition to that, other observers (e.g., Lang and Sawyer [3], Bhatia [5], Karlsson and Litzén [11], and Wagatsuma [15]) report vastly different relative intensities, which are due to the different light sources and spectral registration equipment used. In order to make a comprehensive list of observed lines, the various intensity scales have to be analyzed, and the differences have to be removed to place all intensities on a uniform scale.

One problem with interpreting the In II spectrum is due to wide hfs patterns in most lines observed in the visible range. Indium has two naturally occurring isotopes, ${ }^{113} \mathrm{In}$ and ${ }^{115} \mathrm{In}$, with abundances of $4.3 \%$ and $95.7 \%$, respectively [6]. Both isotopes have the same nuclear spin of $9 / 2$ and similar values of the nuclear magnetic moment, $+5.5289 \mu_{\mathrm{N}}$ and $+5.5408 \mu_{\mathrm{N}}$ [16]. Thus, the hyperfine structures are very similar for both isotopes. The lines from the rare isotope ${ }^{113} \mathrm{In}$ are very hard to observe with samples of natural indium due to their low intensity. In this paper, most of the discussion refers to ${ }^{115} \mathrm{In}$. The hyperfine structure and isotope shifts will be addressed only insofar as they concern the accuracy of the derived center-of-gravity values of energy levels and observed wave numbers of fine-structure transitions.

The aims of the present study are: 1) analyze the measurement uncertainties of different studies, explain and remove inconsistencies, and build a comprehensive list of the best measured wavelengths; 2) from this line list, determine the optimized set of energy levels that fit all observed wavelengths; 3 ) determine an improved value of the ionization limit; 4) analyze line intensities observed by different authors and convert them to a uniform scale; 5) compile a list of recommended values of radiative transition rates. These objectives are addressed in the following sections. 


\section{Evaluation of Measured Wavelengths}

The total list of observed lines compiled in Table 1 includes 680 lines selected from Karlsson and Litzén [11], Larkins and Hannaford [8], von Zanthier et al. [10], Paschen and Campbell [2], Bhatia [5], Lang and Sawyer [3], and Wagatsuma [15]. The first four of these sources provide data that are sufficiently accurate to determine all energy levels involved in other observations. Measurements of Bhatia [5], Lang and Sawyer [3], and Wagatsuma [15] were not included in the level optimization procedure because they were found to possess significant systematic and statistical errors and do not improve the level values.

In Table 1, the wavelengths below $2000 \AA$ are given in vacuum, and above that in standard air. Conversion from vacuum to air and vice versa was made using the five-parameter formula from Peck and Reeder [28]. The uncertainties in the units of the least significant digit of the value are given in parentheses after the value. These uncertainties do not account for the uncertainties of the vacuum-air conversion formula. All uncertainties are meant to be on the level of one standard deviation. Where the systematic uncertainties are significant, they are given in addition to the statistical ones.

A detailed analysis of all observations follows.

\subsection{Measurements of Karlsson and Litzén [11]}

Karlsson and Litzén [11] recorded spectra of indium in the region (12500-55500) $\mathrm{cm}^{-1}$ (i.e., from $8000 \AA$ to $1800 \AA$ ) using a vacuum ultraviolet (VUV) Fourier transform spectrometer. The light source was a hollow cathode discharge with the carrier gas consisting of neon at $170 \mathrm{~Pa}$ (1.3 Torr) or an argon-neon 1:1 mixture at $160 \mathrm{~Pa}$ (1.2 Torr). Small pieces of metallic indium were placed inside the water-cooled iron cathode.

The wavenumber scale was calibrated by means of Ar II lines [17] in the ultraviolet (UV) region, whereas Ne I and Ne II lines were used at longer wavelengths. The neon lines had been measured with high accuracy relative to $\mathrm{Fe}$ I and $\mathrm{Fe}$ II lines [18,19] during previous experiments using an iron cathode and neon as the carrier gas. Since the above-mentioned Fe I-II measurements were calibrated against Ar II wave numbers of Norlén [17], all In II wave numbers measured by Karlsson and Litzén [11] can be ultimately traced to the Ar II reference data of Norlén. It was recently found by Nave and Sansonetti [20] that the wavenumber scale used by Norlén has a calibration error, and all wave numbers from his paper have to be increased by 6.7 parts in $10^{8}$. I applied this correction factor to all In II wave numbers reported in Ref. [11].

To analyze the measurement uncertainties, one has to take into account the hfs of the observed lines, as well as statistical and systematic uncertainties. Karlsson and Litzén noted that most In II lines exhibit wide hfs extending over several reciprocal centimeters. One line at $2941.0375 \AA$ corresponding to the $5 \mathrm{~s} 5 \mathrm{p}{ }^{1} \mathrm{P}^{\circ}{ }_{1}-5 \mathrm{~s} 6 \mathrm{~s}^{1} \mathrm{~S}_{0}$ transition was observed as a symmetric feature with no discernible hfs. This line was denoted as a category ' $a$ ' in Table 1 of Ref. [11] and was fitted with a Voigt profile. Some of the lines have well-resolved hfs with up to 16 distinct components. For these lines, denoted as a category 'c' in [11], Karlsson and Litzén modeled the hfs with an account for the linear and quadratic magnetic hfs and determined the center-of-gravity wave number from this fitting procedure. For lines where the hfs was noticeable but the fitting procedure did not give an unambiguous result due to a lack of resolution or a too low signal-to-noise ratio $(S / N)$, the center of gravity of the observed hfs pattern was derived by fitting the observed feature using an empirical procedure. These lines were denoted as a category ' $b$ ' in Ref. [11].

In the presence of such a large hfs splitting, the positions of centers of gravity of observed features do not necessarily coincide with the differences between centers of gravity of the hfs components of the upper and lower levels, because they depend on the distribution of intensities within the hfs multiplets. This is true even if the population of the hfs sublevels follows the Boltzmann distribution (see the discussion of this effect in relation to the fine structure in the hydrogen spectrum [21]). In such a case, the energy levels derived from the centers of gravity of observed features are only approximate and depend on the observational conditions, which may distort the distribution of intensities and thus shift the centers of gravities of the features. Such approximate energy levels were called distinctive energy levels in [21]. In the case of the In II spectrum, where the hfs is not completely resolved in most lines, only such distinctive energy levels can be determined. They have an intrinsic uncertainty, which is a sizable fraction of the total 
Volume 118 (2013) http://dx.doi.org/10.6028/jres.118.004

Journal of Research of the National Institute of Standards and Technology

Table 1. Observed and predicted spectral lines of In II

\begin{tabular}{|c|c|c|c|c|c|c|c|c|c|c|c|c|}
\hline $\begin{array}{c}\lambda_{\text {obs }}{ }^{\mathrm{a}} \\
(\AA)\end{array}$ & $\begin{array}{c}\sigma_{\mathrm{obs}} \\
\left(\mathrm{cm}^{-1}\right)\end{array}$ & $\begin{array}{c}\lambda_{\text {Ritz }} \mathrm{b} \\
(\AA)\end{array}$ & $\begin{array}{c}\Delta \lambda_{\text {obs-Ritz }}{ }^{c} \\
(\AA)\end{array}$ & $\begin{array}{c}I_{\text {obs }}{ }^{\mathrm{d}} \\
\text { (arb.u.) }\end{array}$ & $\begin{array}{c}A^{\mathrm{e}} \\
\left(\mathrm{s}^{-1}\right)\end{array}$ & Type ${ }^{f}$ & \multicolumn{3}{|c|}{ Transition } & Notes $^{\mathrm{g}}$ & $\begin{array}{l}\text { Line } \\
\text { Ref. }\end{array}$ & $\begin{array}{l}\text { TP } \\
\text { Ref. }^{\mathrm{i}}{ }^{\mathrm{N}}\end{array}$ \\
\hline $680.28(3)(1)$ & 146998 & $680.20(5)$ & 0.08 & 170 & & & $5 s^{2}$ & $5 \mathrm{~s} 12 \mathrm{p}$ & ${ }^{1} \mathrm{P}^{\circ}{ }_{1}$ & & B69 & \\
\hline $686.54(3)(1)$ & 145658 & $686.4185(7)$ & 0.12 & 170 & & & $5 s^{2}$ & ${ }^{1} \mathrm{~S}_{0} \quad 5 \mathrm{~s} 11 \mathrm{p}$ & ${ }^{1} \mathrm{P}_{1}^{\circ}$ & & B69 & \\
\hline 695.83(11)(24) & 143710 & 695.5888(4) & 0.24 & 180 & $6 \mathrm{e}+5$ & $\mathrm{E}$ & $5 s^{2}$ & $5 \mathrm{~s} 10 \mathrm{p}$ & ${ }^{1} \mathrm{P}_{1}^{\circ}$ & 1 & L31 & TW \\
\hline $695.86(3)(0)$ & 143707 & $695.8626(4)$ & 0.00 & 70 & $1.0 \mathrm{e}+5$ & E & $5 \mathrm{~s}^{2}$ & $5 \mathrm{~s} 10 \mathrm{p}$ & ${ }^{3} \mathrm{P}_{1}^{\circ}$ & 1 & B69 & TW \\
\hline $710.09(11)(24)$ & 140830 & $710.00036(18)$ & 0.09 & 95 & $1.5 \mathrm{e}+6$ & E & $5 s^{2}$ & ${ }^{1} \mathrm{~S}_{0} \quad 5 \mathrm{~s} 9 \mathrm{p}$ & ${ }^{1} \mathrm{P}_{1}^{\circ}$ & 1 & L31 & TW \\
\hline $710.55(3)(0)$ & 140736 & $710.55737(21)$ & -0.01 & 47 & $3.1 \mathrm{e}+5$ & $\mathrm{E}$ & $5 s^{2}$ & ${ }^{1} \mathrm{~S}_{0} \quad 5 \mathrm{~s} 9 \mathrm{p}$ & ${ }^{3} \mathrm{P}_{1}^{\circ}$ & 1 & B69 & TW \\
\hline $734.78(3)(0)$ & 136095 & 734.7704(5) & 0.01 & 230 & $3.6 \mathrm{e}+7$ & E & $5 s^{2}$ & ${ }^{1} \mathrm{~S}_{0} \quad 5 \mathrm{~s} 8 \mathrm{p}$ & ${ }^{1} \mathrm{P}_{1}^{\circ}$ & & B69 & B99 \\
\hline $736.04(3)(0)$ & 135862 & $736.0430(3)$ & 0.00 & 210 & $8 \mathrm{e}+5$ & $\mathrm{E}$ & $5 s^{2}$ & ${ }^{1} \mathrm{~S}_{0} \quad 5 \mathrm{~s} 8 \mathrm{p}$ & ${ }^{3} \mathrm{P}_{1}^{\circ}$ & 1 & B69 & TW \\
\hline $783.98(3)(0)$ & 127554 & $783.86296(6)$ & 0.12 & 440 & & & $5 \mathrm{~s}^{2}$ & ${ }^{1} \mathrm{~S}_{0} \quad 5 \mathrm{~s} 7 \mathrm{p}$ & ${ }^{1} \mathrm{P}^{\circ}{ }_{1}$ & & B69 & \\
\hline $787.45(3)(0)$ & 126992 & $787.43326(7)$ & 0.02 & 1100 & $1.7 \mathrm{e}+6$ & E & $5 \mathrm{~s}^{2}$ & $5 \mathrm{~s} 7 \mathrm{p}$ & ${ }^{3} \mathrm{P}_{1}^{\circ}$ & 1 & B69 & TW \\
\hline $910.90(3)(1)$ & 109782 & $910.91090(7)$ & -0.01 & 2700 & $7 \mathrm{e}+6$ & E & $5 s^{2}$ & $5 \mathrm{~s} 6 \mathrm{p}$ & ${ }^{1} \mathrm{P}_{1}^{\circ}$ & & B69 & B99 \\
\hline $927.27(3)(1)$ & 107843 & $927.28257(5)$ & -0.01 & 2500 & $4.9 \mathrm{e}+6$ & $\mathrm{D}^{+}$ & $5 s^{2}$ & $5 \mathrm{~s} 6 \mathrm{p}$ & ${ }^{3} \mathrm{P}_{1}^{\circ}$ & & B69 & TW \\
\hline & & $933.7684(19)$ & & & $1.5 \mathrm{e}+6$ & $\mathrm{D}+$ & $5 \mathrm{~s} 5 \mathrm{p}$ & ${ }^{3} \mathrm{P}^{\circ}{ }_{1} \quad 5 \mathrm{~s} 19 \mathrm{~s}$ & ${ }^{3} \mathrm{~S}_{1}$ & & & TW \\
\hline & & $935.9123(19)$ & & & $1.9 \mathrm{e}+6$ & $\mathrm{D}+$ & $5 \mathrm{~s} 5 \mathrm{p}$ & ${ }^{3} \mathrm{P}_{1}^{\circ} \quad 5 \mathrm{~s} 18 \mathrm{~s}$ & $S_{1}$ & & & TW \\
\hline & & $936.5492(6)$ & & & $1.5 \mathrm{e}+6$ & $\mathrm{D}^{+}$ & $5 \mathrm{~s} 5 \mathrm{p}$ & ${ }^{3} \mathrm{P}_{0}^{\circ} \quad 5 \mathrm{~s} 15 \mathrm{~s}$ & ${ }^{3} \mathrm{~S}_{1}$ & & & TW \\
\hline $937.87(3)(1)$ & 106625 & $937.9023(23)$ & -0.03 & $610^{*}$ & & & $5 \mathrm{~s} 5 \mathrm{p}$ & ${ }^{3} \mathrm{P}_{1}^{\circ} \quad 5 \mathrm{~s} 16 \mathrm{~d}$ & ${ }^{3} \mathrm{D}_{2}$ & & B69 & \\
\hline $937.87(3)(1)$ & 106625 & $937.9269(21)$ & -0.06 & $610^{*}$ & & & $5 \mathrm{~s} 5 \mathrm{p}$ & ${ }^{3} \mathrm{P}_{1}^{\circ} \quad 5 \mathrm{~s} 16 \mathrm{~d}$ & ${ }^{3} \mathrm{D}_{1}$ & & B69 & \\
\hline & & $938.5571(14)$ & & & $2.5 \mathrm{e}+6$ & $\mathrm{D}+$ & $5 \mathrm{~s} 5 \mathrm{p}$ & ${ }^{3} \mathrm{P}^{\circ}{ }_{1} \quad 5 \mathrm{~s} 17 \mathrm{~s}$ & ${ }^{3} \mathrm{~S}_{1}$ & & & TW \\
\hline $940.60(3)(1)$ & 106315 & $940.6123(18)$ & -0.01 & 970 & & & $5 s 5 p$ & ${ }^{3} \mathrm{P}_{0}^{\circ} \quad 5 \mathrm{~s} 13 \mathrm{~d}$ & ${ }^{3} \mathrm{D}_{1}$ & & B69 & \\
\hline $941.87(3)(1)$ & 106172 & $941.8556(15)$ & 0.01 & $760^{*}$ & $3.2 \mathrm{e}+6$ & $\mathrm{D}+$ & $5 \mathrm{~s} 5 \mathrm{p}$ & $5 \mathrm{~s} 16 \mathrm{~s}$ & ${ }^{3} \mathrm{~S}_{1}$ & & B69 & TW \\
\hline $941.87(3)(1)$ & 106172 & $941.9310(6)$ & -0.06 & $760^{*}$ & $2.0 \mathrm{e}+6$ & $\mathrm{D}+$ & $5 \mathrm{~s} 5 \mathrm{p}$ & $5 \mathrm{~s} 14 \mathrm{~s}$ & ${ }^{3} \mathrm{~S}_{1}$ & & B69 & TW \\
\hline $944.81(3)(1)$ & 105841 & $944.7713(20)$ & 0.04 & 1200 & & & $5 \mathrm{~s} 5 \mathrm{p}$ & ${ }^{3} \mathrm{P}^{\circ}{ }_{1} \quad 5 \mathrm{~s} 14 \mathrm{~d}$ & ${ }^{1} \mathrm{D}_{2}$ & & B69 & \\
\hline & & $946.0705(6)$ & & & $4.2 \mathrm{e}+6$ & $\mathrm{D}+$ & $5 \mathrm{~s} 5 \mathrm{p}$ & ${ }^{3} \mathrm{P}_{1}^{\circ} \quad 5 \mathrm{~s} 15 \mathrm{~s}$ & & & & TW \\
\hline $947.41(3)(1)$ & 105551 & $947.3507(7)$ & 0.06 & 390 & & & $5 s 5 p$ & ${ }^{3} \mathrm{P}_{0}^{\circ} \quad 5 \mathrm{~s} 12 \mathrm{~d}$ & ${ }^{3} \mathrm{D}_{1}$ & & B69 & \\
\hline $949.17(3)(1)$ & 105355 & $949.1393(5)$ & 0.03 & 910 & $2.9 \mathrm{e}+6$ & $\mathrm{D}+$ & $5 \mathrm{~s} 5 \mathrm{p}$ & $5 \mathrm{~s} 13 \mathrm{~s}$ & ${ }^{3} \mathrm{~S}_{1}$ & & B69 & TW \\
\hline $950.19(3)(1)$ & 105242 & $950.1936(16)$ & 0.00 & 660 & & & $5 \mathrm{~s} 5 \mathrm{p}$ & $5 \mathrm{~s} 13 \mathrm{~d}$ & ${ }^{3} \mathrm{D}_{2}$ & & B69 & \\
\hline $950.32(3)(1)$ & 105228 & $950.2168(18)$ & 0.10 & 670 & & & $5 \mathrm{~s} 5 \mathrm{p}$ & $5 \mathrm{~s} 13 \mathrm{~d}$ & ${ }^{3} \mathrm{D}_{1}$ & & B69 & \\
\hline $951.27(3)(1)$ & 105123 & $951.2487(20)$ & 0.02 & 1200 & & & $5 \mathrm{~s} 5 \mathrm{p}$ & $5 \mathrm{~s} 14 \mathrm{~s}$ & ${ }^{1} \mathrm{~S}_{0}$ & & B69 & \\
\hline
\end{tabular}


Volume 118 (2013) http://dx.doi.org/10.6028/jres.118.004

Journal of Research of the National Institute of Standards and Technology

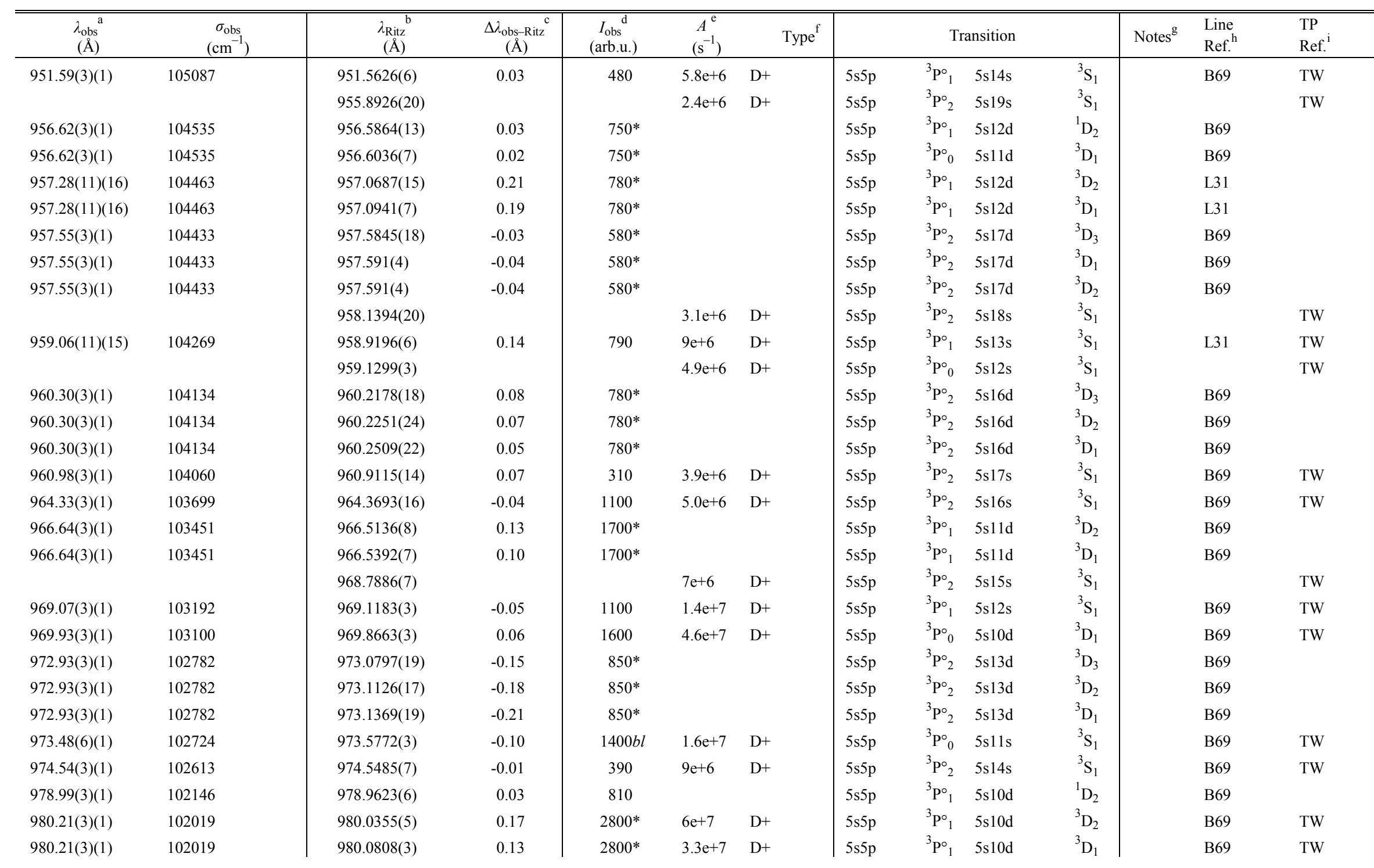


Volume 118 (2013) http://dx.doi.org/10.6028/jres.118.004

Journal of Research of the National Institute of Standards and Technology

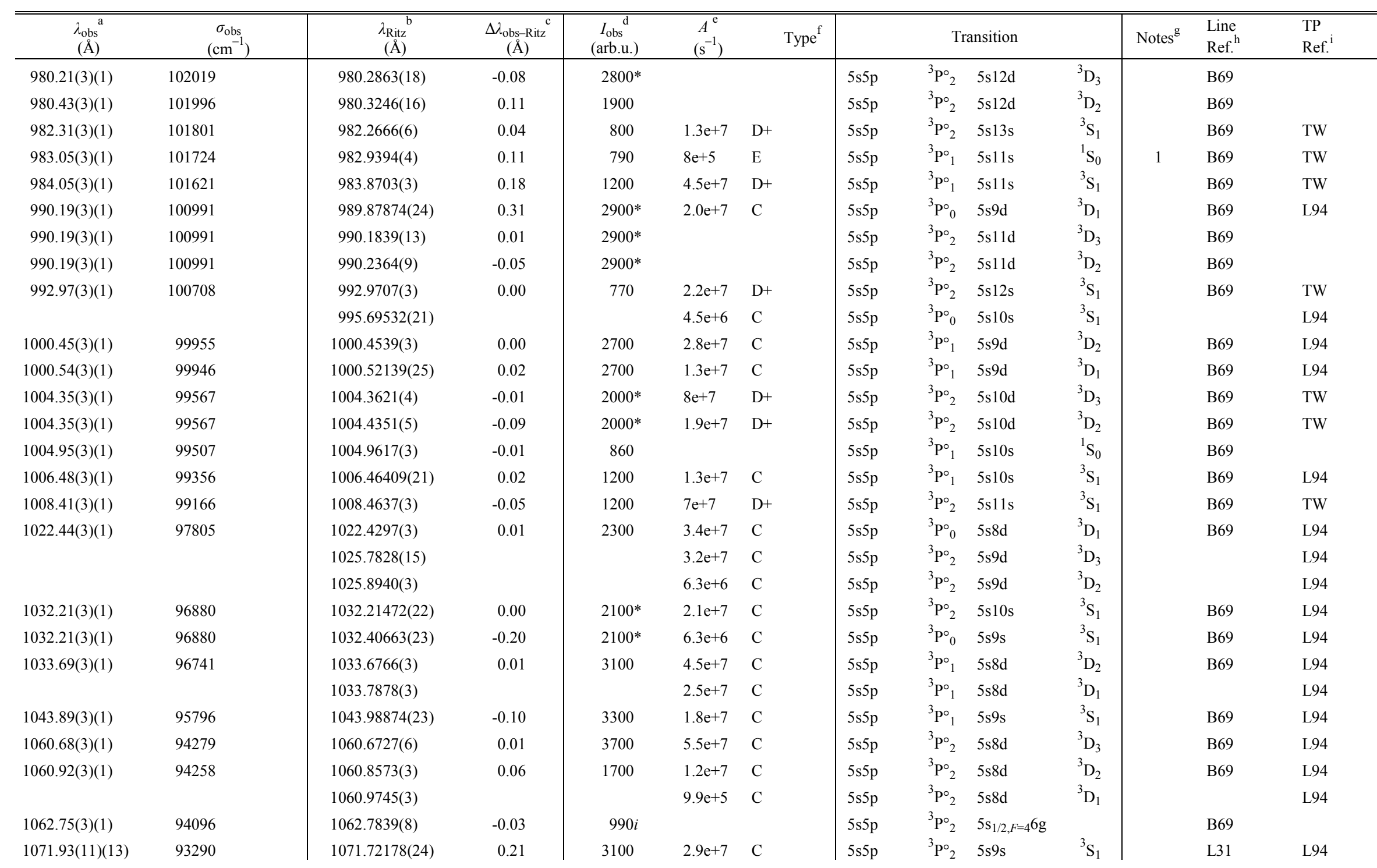


Volume 118 (2013) http://dx.doi.org/10.6028/jres.118.004

Journal of Research of the National Institute of Standards and Technology

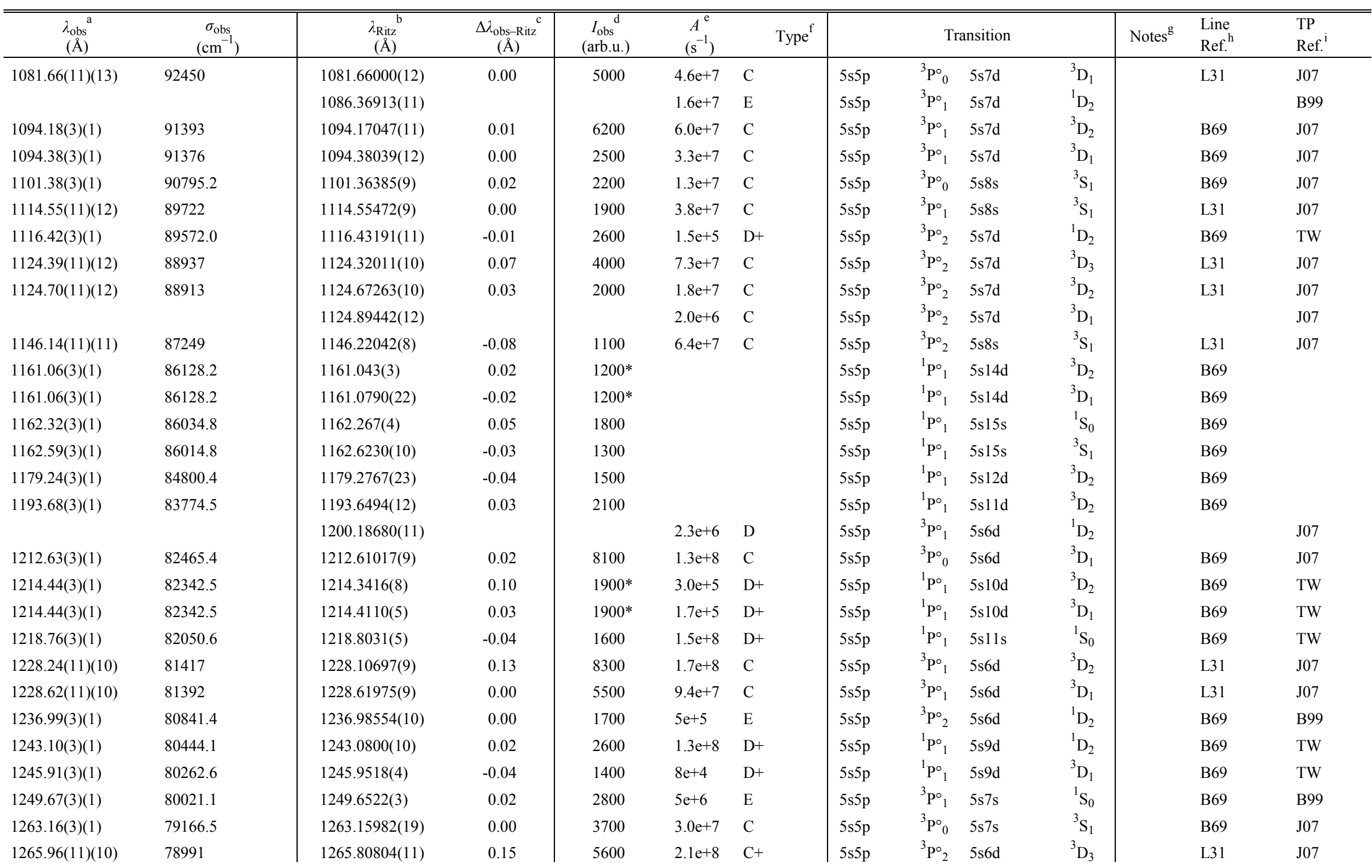


Volume 118 (2013) http://dx.doi.org/10.6028/jres.118.004

Journal of Research of the National Institute of Standards and Technology

\begin{tabular}{|c|c|c|c|c|c|c|c|c|c|c|c|c|}
\hline $\begin{array}{c}\lambda_{\text {obs }}{ }^{\mathrm{a}} \\
(\AA)\end{array}$ & $\begin{array}{c}\sigma_{\mathrm{obs}} \\
\left(\mathrm{cm}^{-1}\right)\end{array}$ & $\begin{array}{c}\lambda_{\text {Ritz }}{ }^{b} \\
(\AA) \\
\end{array}$ & $\begin{array}{c}\Delta \lambda_{\text {obs-Ritz }}{ }^{c} \\
(\AA)\end{array}$ & $\begin{array}{c}I_{\mathrm{obs}}^{\mathrm{d}} \\
\text { (arb.u.) }\end{array}$ & $\begin{array}{l}A^{\mathrm{e}} \\
\left(\mathrm{s}^{-1}\right)\end{array}$ & Type $^{f}$ & \multicolumn{3}{|c|}{ Transition } & Notes $^{\mathrm{g}}$ & $\begin{array}{l}\text { Line } \\
\text { Ref. }^{h}\end{array}$ & $\begin{array}{l}\text { TP } \\
\text { Ref. }^{\text {i }}\end{array}$ \\
\hline 1266.67(3)(1) & 78947.2 & $1266.66524(7)$ & 0.00 & 4900 & $5.2 \mathrm{e}+7$ & $\mathrm{C}$ & $5 \mathrm{~s} 5 \mathrm{p}$ & ${ }^{3} \mathrm{P}^{\circ}{ }_{2} \quad 5 \mathrm{~s} 6 \mathrm{~d}$ & ${ }^{3} \mathrm{D}_{2}$ & & B69 & J07 \\
\hline $1267.19(11)(9)$ & 78915 & $1267.21073(8)$ & -0.02 & 1400 & $5.8 \mathrm{e}+6$ & $\mathrm{C}$ & $5 \mathrm{~s} 5 \mathrm{p}$ & ${ }^{3} \mathrm{P}^{\circ}{ }_{2} \quad 5 \mathrm{~s} 6 \mathrm{~d}$ & ${ }^{3} \mathrm{D}_{1}$ & & $\mathrm{~L} 31$ & $\mathrm{~J} 07$ \\
\hline $1280.49(11)(9)$ & 78095 & $1280.54157(20)$ & -0.05 & 28000 & $8.9 \mathrm{e}+7$ & $\mathrm{C}$ & $5 \mathrm{~s} 5 \mathrm{p}$ & ${ }^{3} \mathrm{P}_{1}^{\circ} \quad 5 \mathrm{~s} 7 \mathrm{~s}$ & ${ }^{3} \mathrm{~S}_{1}$ & & $\mathrm{~L} 31$ & $\mathrm{~J} 07$ \\
\hline & & $1283.0555(5)$ & & & $8 \mathrm{e}+6$ & $\mathrm{D}$ & $5 \mathrm{~s} 5 \mathrm{p}$ & ${ }^{3} \mathrm{P}_{1}^{\circ} \quad 5 \mathrm{p}^{2}$ & & & & J07 \\
\hline $1292.50(3)(1)$ & 77369.4 & $1292.4937(7)$ & 0.01 & 2000 & $1.7 \mathrm{e}+8$ & $\mathrm{D}+$ & $5 \mathrm{~s} 5 \mathrm{p}$ & ${ }^{1} \mathrm{P}_{1}^{\circ} \quad 5 \mathrm{~s} 8 \mathrm{~d}$ & ${ }^{1} \mathrm{D}_{2}$ & & B69 & TW \\
\hline $1297.78(3)(1)$ & 77054.7 & $1297.7896(5)$ & -0.01 & 1000 & & & $5 \mathrm{~s} 5 \mathrm{p}$ & ${ }^{1} \mathrm{P}_{1}^{\circ} \quad 5 \mathrm{~s} 8 \mathrm{~d}$ & ${ }^{3} \mathrm{D}_{2}$ & & B69 & \\
\hline 1309.82(3)(1) & 76346.4 & 1309.7791(5) & 0.04 & 2200 & $2.4 \mathrm{e}+7$ & E & $5 \mathrm{~s} 5 \mathrm{p}$ & ${ }^{1} \mathrm{P}_{1}^{\circ} \quad 5 \mathrm{~s} 9 \mathrm{~s}$ & & 1 & B69 & TW \\
\hline 1314.12(3)(1) & 76096.6 & $1314.0862(4)$ & 0.03 & 3 & $1.1 \mathrm{e}+5$ & $\mathrm{D}+$ & $5 \mathrm{~s} 5 \mathrm{p}$ & ${ }^{1} \mathrm{P}^{\circ}{ }_{1} \quad 5 \mathrm{~s} 9 \mathrm{~s}$ & & & B69 & TW \\
\hline 1322.51(3)(1) & 75613.8 & $1322.51892(20)$ & -0.01 & 4200 & $1.5 \mathrm{e}+8$ & $\mathrm{C}$ & $5 \mathrm{~s} 5 \mathrm{p}$ & ${ }^{3} \mathrm{P}_{2}^{\circ} \quad 5 \mathrm{~s} 7 \mathrm{~s}$ & & & B69 & $\mathrm{J} 07$ \\
\hline & & $1381.94502(20)$ & & & $1.8 \mathrm{e}+8$ & $\mathrm{D}$ & $5 \mathrm{~s} 5 \mathrm{p}$ & ${ }^{1} \mathrm{P}_{1}^{\circ} \quad 5 \mathrm{~s} 7 \mathrm{~d}$ & ${ }^{1} \mathrm{D}_{2}$ & & & J07 \\
\hline $1394.92(3)(0)$ & 71688.7 & $1394.93473(23)$ & -0.01 & 190 & $2.4 \mathrm{e}+5$ & $\mathrm{D}+$ & $5 \mathrm{~s} 5 \mathrm{p}$ & ${ }^{1} \mathrm{P}_{1}^{\circ} \quad 5 \mathrm{~s} 7 \mathrm{~d}$ & ${ }^{3} \mathrm{D}_{1}$ & & B69 & TW \\
\hline $1417.75(3)(0)$ & 70534.3 & $1417.74920(22)$ & 0.00 & 410 & $3.6 \mathrm{e}+6$ & $\mathrm{E}$ & $5 \mathrm{~s} 5 \mathrm{p}$ & ${ }^{3} \mathrm{P}_{1}^{\circ} \quad 5 \mathrm{p}^{2}$ & ${ }^{1} \mathrm{D}_{2}$ & & B69 & B99 \\
\hline $1418.13(3)(0)$ & 70515.4 & $1418.1212(3)$ & 0.01 & 2000 & $1.2 \mathrm{e}+8$ & $\mathrm{D}$ & $5 \mathrm{~s} 5 \mathrm{p}$ & ${ }^{1} \mathrm{P}^{\circ}{ }_{1} \quad 5 \mathrm{~s} 8 \mathrm{~s}$ & ${ }^{1} \mathrm{~S}_{0}$ & & B69 & $\mathrm{J} 07$ \\
\hline $1469.49(11)(8)$ & 68051 & $1469.38544(22)$ & 0.10 & 2300 & $2.5 \mathrm{e}+7$ & $\mathrm{D}$ & $5 \mathrm{~s} 5 \mathrm{p}$ & ${ }^{3} \mathrm{P}_{2}^{\circ} \quad 5 \mathrm{p}^{2}$ & ${ }^{1} \mathrm{D}_{2}$ & & L31 & $\mathrm{J} 07$ \\
\hline $1571.53(3)(0)$ & 63632.3 & $1571.52648(23)$ & 0.00 & 260000 & $5.2 \mathrm{e}+8$ & B & $5 s 5 p$ & ${ }^{1} \mathrm{P}^{\circ}{ }_{1} \quad 5 \mathrm{~s} 6 \mathrm{~d}$ & ${ }^{1} \mathrm{D}_{2}$ & & B69 & J07 \\
\hline $1586.37(11)(7)$ & 63037 & $1586.33100(24)$ & 0.04 & 850000 & $1.28 \mathrm{e}+9$ & $\mathrm{~B}+$ & $5 s^{2}$ & ${ }^{1} \mathrm{~S}_{0} \quad 5 \mathrm{~s} 5 \mathrm{p}$ & ${ }^{1} \mathrm{P}^{\circ}{ }_{1}$ & & L31 & CMEF00 \\
\hline $1607.32(3)(1)$ & 62215.4 & $1607.3371(3)$ & -0.02 & 260000 & $3.2 \mathrm{e}+8$ & B & $5 \mathrm{~s} 5 \mathrm{p}$ & ${ }^{3} \mathrm{P}_{1}^{\circ} \quad 5 \mathrm{p}^{2}$ & ${ }^{3} \mathrm{P}_{2}$ & & B69 & $\mathrm{J} 07$ \\
\hline 1619.80(3)(1) & 61736.0 & $1619.74357(23)$ & 0.06 & 17 & $2.7 \mathrm{e}+5$ & $\mathrm{E}$ & $5 \mathrm{~s} 5 \mathrm{p}$ & ${ }^{1} \mathrm{P}_{1}^{\circ} \quad 5 \mathrm{~s} 6 \mathrm{~d}$ & ${ }^{3} \mathrm{D}_{2}$ & 1 & B69 & TW \\
\hline $1620.60(3)(1)$ & 61705.5 & $1620.63567(23)$ & -0.04 & 15 & $5.2 \mathrm{e}+5$ & $\mathrm{D}+$ & $5 \mathrm{~s} 5 \mathrm{p}$ & ${ }^{1} \mathrm{P}_{1}^{\circ} \quad 5 \mathrm{~s} 6 \mathrm{~d}$ & ${ }^{3} \mathrm{D}_{1}$ & & B69 & TW \\
\hline $1640.05(3)(1)$ & 60973.8 & $1640.060(4)$ & -0.01 & 180000 & $3.7 \mathrm{e}+8$ & $\mathrm{C}$ & $5 \mathrm{~s} 5 \mathrm{p}$ & ${ }^{3} \mathrm{P}_{0}^{\circ} \quad 5 \mathrm{p}^{2}$ & & & B69 & $\mathrm{J} 07$ \\
\hline & & $1657.4320(5)$ & & 240000 & $1.36 \mathrm{e}+9$ & B & $5 \mathrm{~s} 5 \mathrm{p}$ & ${ }^{1} \mathrm{P}_{1}^{\circ} \quad 5 \mathrm{~s} 7 \mathrm{~s}$ & ${ }^{1} \mathrm{~S}_{0}$ & & W96 & J07 \\
\hline $1669.46(3)(1)$ & 59899.6 & $1669.482(4)$ & -0.02 & 110000 & $3.5 \mathrm{e}+8$ & $\mathrm{C}$ & $5 \mathrm{~s} 5 \mathrm{p}$ & ${ }^{3} \mathrm{P}_{1}^{\circ} \quad 5 \mathrm{p}^{2}$ & ${ }^{3} \mathrm{P}_{1}$ & & B69 & $\mathrm{J} 07$ \\
\hline 1671.87(3)(1) & 59813.3 & $1671.8850(11)$ & -0.02 & 120000 & $7.3 \mathrm{e}+8$ & B & $5 \mathrm{~s} 5 \mathrm{p}$ & ${ }^{3} \mathrm{P}_{0}^{\circ} \quad 5 \mathrm{~s} 5 \mathrm{~d}$ & ${ }^{3} \mathrm{D}_{1}$ & & B69 & A86c \\
\hline $1674.02(3)(1)$ & 59736.4 & $1674.0317(3)$ & -0.01 & 130000 & $9.5 \mathrm{e}+8$ & B & $5 \mathrm{~s} 5 \mathrm{p}$ & ${ }^{3} \mathrm{P}_{2}^{\circ} \quad 5 \mathrm{p}^{2}$ & ${ }^{3} \mathrm{P}_{2}$ & & B69 & A86c,J07 \\
\hline 1699.97(3)(1) & 58824.6 & $1699.9833(7)$ & -0.01 & 200000 & $8.6 \mathrm{e}+8$ & B & $5 \mathrm{~s} 5 \mathrm{p}$ & ${ }^{3} \mathrm{P}_{1}^{\circ} \quad 5 \mathrm{~s} 5 \mathrm{~d}$ & ${ }^{3} \mathrm{D}_{2}$ & & B69 & A86c \\
\hline $1702.45(3)(1)$ & 58738.9 & $1702.4714(12)$ & -0.02 & 120000 & $4.1 \mathrm{e}+8$ & B & $5 \mathrm{~s} 5 \mathrm{p}$ & ${ }^{3} \mathrm{P}^{\circ}{ }_{1} \quad 5 \mathrm{~s} 5 \mathrm{~d}$ & ${ }^{3} \mathrm{D}_{1}$ & & B69 & $\mathrm{J} 07$ \\
\hline $1716.53(3)(1)$ & 58257.1 & $1716.518(6)$ & 0.01 & 110000 & $1.1 \mathrm{e}+9$ & $\mathrm{C}$ & $5 \mathrm{~s} 5 \mathrm{p}$ & ${ }^{3} \mathrm{P}_{1}^{\circ} \quad 5 \mathrm{p}^{2}$ & ${ }^{3} \mathrm{P}_{0}$ & & B69 & $\mathrm{J} 07$ \\
\hline 1716.70(3)(1) & 58251.3 & $1716.7092(9)$ & -0.01 & 19000 & $1.08 \mathrm{e}+9$ & $\mathrm{C}+$ & $5 \mathrm{~s} 5 \mathrm{p}$ & ${ }^{1} \mathrm{P}^{\circ}{ }_{1} \quad 5 \mathrm{p}^{2}$ & ${ }^{1} \mathrm{~S}_{0}$ & & B69 & A86c \\
\hline 1741.53(3)(1) & 57420.8 & $1741.549(4)$ & -0.02 & 95000 & $4.9 \mathrm{e}+8$ & B & $5 \mathrm{~s} 5 \mathrm{p}$ & ${ }^{3} \mathrm{P}_{2}^{\circ} \quad 5 \mathrm{p}^{2}$ & ${ }^{3} \mathrm{P}_{1}$ & & B69 & $\mathrm{J} 07$ \\
\hline $1770.56(3)(1)$ & 56479.3 & $1770.5652(4)$ & -0.01 & 140000 & $1.06 \mathrm{e}+9$ & A & $5 \mathrm{~s} 5 \mathrm{p}$ & ${ }^{3} \mathrm{P}_{2}^{\circ} \quad 5 \mathrm{~s} 5 \mathrm{~d}$ & ${ }^{3} \mathrm{D}_{3}$ & & B69 & A86 \\
\hline
\end{tabular}


Volume 118 (2013) http://dx.doi.org/10.6028/jres.118.004

Journal of Research of the National Institute of Standards and Technology

\begin{tabular}{|c|c|c|c|c|c|c|c|c|c|c|c|c|}
\hline $\begin{array}{l}\lambda_{\text {obs }}{ }^{a} \\
(\AA)\end{array}$ & $\begin{array}{c}\sigma_{\mathrm{obs}} \\
\left(\mathrm{cm}^{-1}\right)\end{array}$ & $\begin{array}{c}\lambda_{\text {Ritz }} \text { b } \\
(\AA)\end{array}$ & $\begin{array}{c}\Delta \lambda_{\text {obs-Ritz }}{ }^{c} \\
(\AA)\end{array}$ & $\begin{array}{c}I_{\mathrm{obs}}^{\mathrm{d}} \\
\text { (arb.u.) }\end{array}$ & $\begin{array}{l}A^{\mathrm{e}} \\
\left(\mathrm{s}^{-1}\right)\end{array}$ & Type $^{\mathrm{f}}$ & & Transition & & Notes $^{\mathrm{g}}$ & $\begin{array}{l}\text { Line } \\
\text { Ref. }^{h}\end{array}$ & $\begin{array}{l}\text { TP } \\
\text { Ref. }^{\text {i }}\end{array}$ \\
\hline 1774.75(3)(1) & 56346.0 & $1774.7667(8)$ & -0.02 & 58000 & $2.34 \mathrm{e}+8$ & B & $5 \mathrm{~s} 5 \mathrm{p}$ & ${ }^{3} \mathrm{P}_{2}^{\circ}$ & ${ }^{3} \mathrm{D}_{2}$ & & B69 & J07 \\
\hline $1777.46(3)(1)$ & 56260.1 & $1777.4787(13)$ & -0.02 & 27000 & $1.6 \mathrm{e}+7$ & $\mathrm{C}$ & $5 \mathrm{~s} 5 \mathrm{p}$ & $5 \mathrm{~s} 5 \mathrm{~d}$ & ${ }^{3} \mathrm{D}_{1}$ & & B69 & J07 \\
\hline $1842.36(3)(1)$ & 54278.2 & $1842.3720(3)$ & -0.01 & 26000 & $3.7 \mathrm{e}+6$ & $\mathrm{D}$ & $5 \mathrm{~s} 5 \mathrm{p}$ & $5 \mathrm{~s} 5 \mathrm{~d}$ & ${ }^{1} \mathrm{D}_{2}$ & & B69 & J07 \\
\hline 1862.89(3)(1) & 53680.0 & $1862.9040(4)$ & -0.01 & 4900 & $4.7 \mathrm{e}+6$ & $\mathrm{D}$ & $5 \mathrm{~s} 5 \mathrm{p}$ & ${ }^{3} \mathrm{P}^{\circ}{ }_{1}$ & ${ }^{1} \mathrm{~S}_{0}$ & & B69 & J07 \\
\hline 1930.5331(4) & 51799.164 & $1930.5325(3)$ & 0.0006 & 25000 & $1.1 \mathrm{e}+7$ & $\mathrm{D}+$ & $5 \mathrm{~s} 5 \mathrm{p}$ & $5 \mathrm{~s} 5 \mathrm{~d}$ & & & $\mathrm{~K} 01 \mathrm{c}$ & J07 \\
\hline 1932.14(9)(1) & 51756.1 & $1932.000(5)$ & 0.14 & 590 & & & $5 \mathrm{~s} 6 \mathrm{~s}$ & ${ }^{3} \mathrm{~S}_{1} \quad 5 \mathrm{~s} 11 \mathrm{p}$ & ${ }^{1} \mathrm{P}^{\circ}{ }_{1}$ & & B69 & \\
\hline 1936.1876(3) & 51647.888 & $1936.18758(18)$ & 0.0000 & 20000 & $9.6 \mathrm{e}+7$ & $\mathrm{C}$ & $5 \mathrm{~s} 5 \mathrm{p}$ & $5 \mathrm{~s} 6 \mathrm{~s}$ & & & $\mathrm{~K} 01 \mathrm{c}$ & J07 \\
\hline 1953.34(9)(1) & 51194.4 & $1953.434(5)$ & -0.09 & 300 & & & $5 \mathrm{~s} 6 \mathrm{~s}$ & ${ }^{3} \mathrm{~S}_{1} \quad 5 \mathrm{~s} 8 \mathrm{f}$ & ${ }^{3} \mathrm{~F}_{2}^{\circ}$ & & B69 & \\
\hline 1965.98(9)(1) & 50865.2 & 1966.063(5) & -0.08 & 720 & & & $5 \mathrm{~s} 5 \mathrm{~d}$ & $5 \mathrm{~s} 11 \mathrm{f}$ & ${ }^{1} \mathrm{~F}_{3}^{\circ}$ & 2 & B69 & \\
\hline 1966.7087(3) & 50846.372 & $1966.70862(24)$ & 0.0001 & 92000 & $1.27 \mathrm{e}+9$ & $\mathrm{~B}+$ & $5 \mathrm{~s} 5 \mathrm{p}$ & ${ }^{1} \mathrm{P}_{1}^{\circ}$ & ${ }^{1} \mathrm{D}_{2}$ & & $\mathrm{~K} 01 \mathrm{c}$ & A86c \\
\hline $1977.32788(23)$ & 50573.302 & $1977.32789(19)$ & -0.00001 & 32000 & $2.7 \mathrm{e}+8$ & $\mathrm{~B}$ & $5 \mathrm{~s} 5 \mathrm{p}$ & $5 \mathrm{~s} 6 \mathrm{~s}$ & ${ }^{3} \mathrm{~S}_{1}$ & & K01c & $\mathrm{J} 07$ \\
\hline 1997.03(9)(1) & 50074.4 & 1996.968(5) & 0.06 & 240 & $1.5 \mathrm{e}+7$ & $\mathrm{D}+$ & $5 \mathrm{~s} 5 \mathrm{~d}$ & $5 \mathrm{~s} 10 \mathrm{f}$ & ${ }^{1} \mathrm{~F}_{3}^{\circ}$ & & B69 & TW \\
\hline 1998.04(9)(1) & 50049.0 & 1997.921(5) & 0.12 & 140 & & & $5 \mathrm{~s} 5 \mathrm{~d}$ & $5 \mathrm{~s} 10 \mathrm{f}$ & ${ }^{3} \mathrm{~F}_{3}^{\circ}$ & & B69 & \\
\hline 2008.12(9)(1) & 49781.7 & 2008.082(3) & 0.04 & 140 & & & $5 \mathrm{~s} 6 \mathrm{~s}$ & $5 \mathrm{~s} 10 \mathrm{p}$ & & & B69 & \\
\hline 2039.79(9)(1) & 49008.9 & $2039.748(5)$ & 0.04 & 330 & $4.0 \mathrm{e}+7$ & $\mathrm{D}+$ & $5 \mathrm{~s} 5 \mathrm{~d}$ & $5 \mathrm{~s} 9 \mathrm{f}$ & ${ }^{1} \mathrm{~F}_{3}^{\circ}$ & & B69 & TW \\
\hline 2040.81(9)(1) & 48984.4 & $2040.7532(16)$ & 0.06 & $210^{*}$ & & & $5 \mathrm{~s} 5 \mathrm{~d}$ & $5 \mathrm{~s} 9 \mathrm{f}$ & ${ }^{3} \mathrm{~F}_{2}^{\circ}$ & & B69 & \\
\hline 2040.81(9)(1) & 48984.4 & $2040.834(5)$ & -0.02 & $210^{*}$ & & & $5 \mathrm{~s} 5 \mathrm{~d}$ & $5 \mathrm{~s} 9 \mathrm{f}$ & ${ }^{3} \mathrm{~F}_{3}^{\circ}$ & & B69 & \\
\hline 2054.76(9)(1) & 48651.9 & 2054.692(6) & 0.07 & 210 & & & $5 \mathrm{~s} 6 \mathrm{~s}$ & ${ }^{1} \mathrm{~S}_{0} \quad 5 \mathrm{~s} 11 \mathrm{p}$ & ${ }^{1} \mathrm{P}_{1}^{\circ}$ & & B69 & \\
\hline 2055.72(9)(1) & 48629.2 & $2055.872(7)$ & -0.15 & 400 & & & $5 \mathrm{~s} 6 \mathrm{~s}$ & $5 \mathrm{~s} 11 \mathrm{p}$ & ${ }^{3} \mathrm{P}_{1}^{\circ}$ & 2 & B69 & \\
\hline $2078.57182(13)$ & 48094.629 & $2078.57186(13)$ & -0.00004 & 76000 & $4.2 \mathrm{e}+8$ & $\mathrm{~B}$ & $5 \mathrm{~s} 5 \mathrm{p}$ & $5 \mathrm{~s} 6 \mathrm{~s}$ & & & $\mathrm{~K} 01 \mathrm{c}$ & A86c,J07 \\
\hline $2080.30(9)(1)$ & 48054.7 & $2080.274(6)$ & 0.03 & 330 & & & $5 \mathrm{~s} 5 \mathrm{~d}$ & ${ }^{1} \mathrm{D}_{2} \quad 5 \mathrm{~s} 11 \mathrm{p}$ & ${ }^{1} \mathrm{P}_{1}^{\circ}$ & & B69 & \\
\hline 2081.51(9)(1) & 48026.7 & $2081.483(7)$ & 0.03 & 300 & & & $5 \mathrm{~s} 5 \mathrm{~d}$ & $5 \mathrm{~s} 11 \mathrm{p}$ & ${ }^{3} \mathrm{P}_{1}^{\circ}$ & & B69 & \\
\hline 2103.891(18) & 47515.9 & $2103.886(5)$ & 0.005 & 1700 & $1.0 \mathrm{e}+7$ & E & $5 \mathrm{~s} 5 \mathrm{~d}$ & $5 \mathrm{~s} 8 \mathrm{f}$ & ${ }^{1} \mathrm{~F}_{3}^{\circ}$ & 1 & P38P & TW \\
\hline 2105.10(9)(1) & 47488.6 & $2105.076(4)$ & 0.02 & $190 *$ & & & $5 \mathrm{~s} 5 \mathrm{~d}$ & $5 \mathrm{~s} 8 \mathrm{f}$ & ${ }^{3} \mathrm{~F}_{3}^{\circ}$ & & B69 & \\
\hline 2105.10(9)(1) & 47488.6 & $2105.156(6)$ & -0.06 & $190^{*}$ & & & $5 \mathrm{~s} 5 \mathrm{~d}$ & $5 \mathrm{~s} 8 \mathrm{f}$ & ${ }^{3} \mathrm{~F}_{2}^{\circ}$ & & B69 & \\
\hline 2135.64(9)(1) & 46809.6 & $2135.5898(19)$ & 0.05 & 160 & $3.5 \mathrm{e}+5$ & E & $5 \mathrm{~s} 6 \mathrm{~s}$ & ${ }^{3} \mathrm{~S}_{1} \quad 5 \mathrm{~s} 9 \mathrm{p}$ & ${ }^{3} \mathrm{P}^{\circ}{ }_{1}$ & 1 & B69 & TW \\
\hline 2139.150(18) & 46732.8 & $2139.146(4)$ & 0.004 & 540 & & & $5 \mathrm{~s} 6 \mathrm{~s}$ & ${ }^{1} \mathrm{~S}_{0} \quad 5 \mathrm{~s} 10 \mathrm{p}$ & ${ }^{1} \mathrm{P}^{\circ}{ }_{1}$ & & P38P & \\
\hline 2141.68(9)(1) & 46677.6 & 2141.739(4) & -0.06 & 170 & & & $5 \mathrm{~s} 6 \mathrm{~s}$ & ${ }^{1} \mathrm{~S}_{0} \quad 5 \mathrm{~s} 10 \mathrm{p}$ & ${ }^{3} \mathrm{P}^{\circ}{ }_{1}$ & & B69 & \\
\hline 2166.876(19) & 46134.9 & $2166.888(4)$ & -0.012 & 990 & $1.2 \mathrm{e}+7$ & $\mathrm{D}+$ & $5 \mathrm{~s} 5 \mathrm{~d}$ & ${ }^{1} \mathrm{D}_{2} \quad 5 \mathrm{~s} 10 \mathrm{p}$ & ${ }^{1} \mathrm{P}_{1}^{\circ}$ & & P38P & TW \\
\hline 2169.548(19) & 46078.1 & $2169.548(4)$ & 0.000 & 560 & $1.7 \mathrm{e}+6$ & $\mathrm{D}^{+}$ & $5 \mathrm{~s} 5 \mathrm{~d}$ & ${ }^{1} \mathrm{D}_{2} \quad 5 \mathrm{~s} 10 \mathrm{p}$ & ${ }^{3} \mathrm{P}_{1}^{\circ}$ & & P38P & TW \\
\hline
\end{tabular}


Volume 118 (2013) http://dx.doi.org/10.6028/jres.118.004

Journal of Research of the National Institute of Standards and Technology

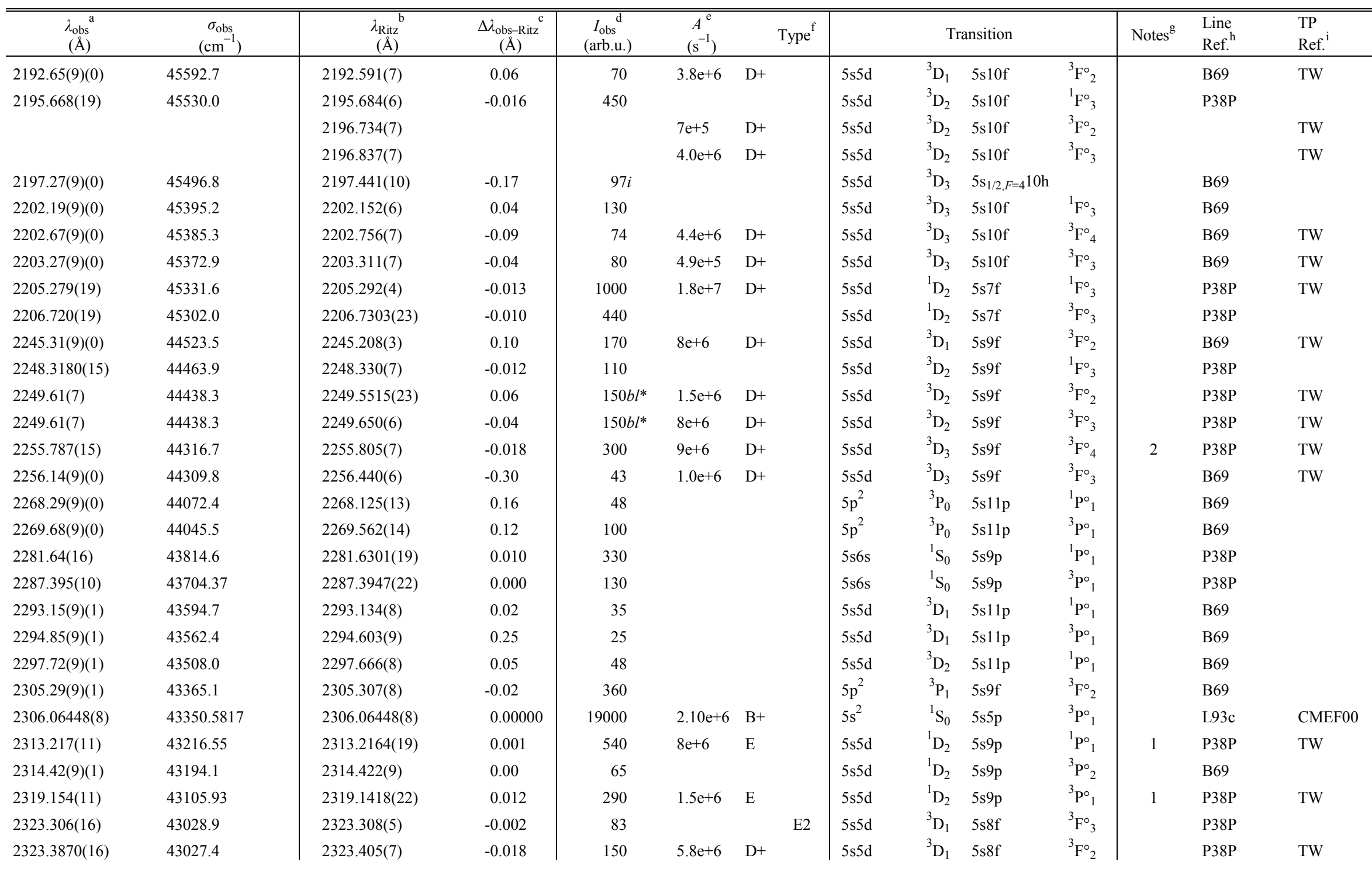


Volume 118 (2013) http://dx.doi.org/10.6028/jres.118.004

Journal of Research of the National Institute of Standards and Technology

\begin{tabular}{|c|c|c|c|c|c|c|c|c|c|c|c|c|c|}
\hline $\begin{array}{c}\lambda_{\text {obs }}{ }^{\mathrm{a}} \\
(\AA)\end{array}$ & $\begin{array}{c}\begin{array}{c}\sigma_{\mathrm{obs}} \\
\left(\mathrm{cm}^{-1}\right)\end{array} \\
\end{array}$ & $\begin{array}{c}\lambda_{\text {Ritz }}{ }^{b} \\
(\AA)\end{array}$ & $\begin{array}{c}\Delta \lambda_{\text {obs-Ritz }}{ }^{c} \\
(\AA)\end{array}$ & $\begin{array}{c}I_{\text {obs }}{ }^{\mathrm{d}} \\
\text { (arb.u.) }\end{array}$ & $\begin{array}{l}A^{\mathrm{e}} \\
\left(\mathrm{s}^{-1}\right)\end{array}$ & & Type $^{f}$ & \multicolumn{3}{|c|}{ Transition } & Notes $^{\mathrm{g}}$ & $\begin{array}{l}\text { Line } \\
\text { Ref. }^{h}\end{array}$ & $\begin{array}{l}\text { TP } \\
\text { Ref. }^{i}\end{array}$ \\
\hline $2326.4910(16)$ & 42970.0 & $2326.504(6)$ & -0.013 & 67 & & & & $5 \mathrm{~s} 5 \mathrm{~d}$ & ${ }^{3} \mathrm{D}_{2} \quad 5 \mathrm{~s} 8 \mathrm{f}$ & ${ }^{1} \mathrm{~F}_{3}^{\circ}$ & & P38P & \\
\hline $2327.949(11)$ & 42943.08 & $2327.959(5)$ & -0.010 & 290 & $6 \mathrm{e}+6$ & $\mathrm{D}+$ & & $5 \mathrm{~s} 5 \mathrm{~d}$ & ${ }^{3} \mathrm{D}_{2} \quad 5 \mathrm{~s} 8 \mathrm{f}$ & & & P38P & TW \\
\hline & & $2328.057(7)$ & & & $1.1 \mathrm{e}+6$ & D+ & & $5 \mathrm{~s} 5 \mathrm{~d}$ & ${ }^{3} \mathrm{D}_{2} \quad 5 \mathrm{~s} 8 \mathrm{f}$ & & & & TW \\
\hline 2334.570(16) & 42821.3 & 2334.584(9) & -0.014 & 320 & $7 e+6$ & $\mathrm{D}+$ & & $5 \mathrm{~s} 5 \mathrm{~d}$ & ${ }^{3} \mathrm{D}_{3} \quad 5 \mathrm{~s} 8 \mathrm{f}$ & & & P38P & TW \\
\hline & & 2335.231(5) & & & $8 \mathrm{e}+5$ & $\mathrm{D}+$ & & $5 \mathrm{~s} 5 \mathrm{~d}$ & ${ }^{3} \mathrm{D}_{3} \quad 5 \mathrm{~s} 8 \mathrm{f}$ & & & & TW \\
\hline 2343.49(9)(1) & 42658.3 & $2343.298(12)$ & 0.19 & 310 & & & & $5 \mathrm{~s} 6 \mathrm{p}$ & ${ }^{3} \mathrm{P}_{1}^{\circ} \quad 5 \mathrm{~s} 18 \mathrm{~d}$ & & & B69 & \\
\hline $2343.87(3)$ & 42651.5 & $2343.865(16)$ & 0.01 & $89^{*}$ & & & E2 & $5 \mathrm{~s} 6 \mathrm{p}$ & ${ }^{3} \mathrm{P}_{1}^{\circ} \quad 5 \mathrm{~s} 18 \mathrm{~d}$ & & & P38P & \\
\hline 2343.87(3) & 42651.5 & $2343.865(23)$ & & $89^{*}$ & & & & $5 \mathrm{~s} 6 \mathrm{p}$ & ${ }^{3} \mathrm{P}^{\circ}{ }_{1} \quad 5 \mathrm{~s} 18 \mathrm{~d}$ & & & P38P & \\
\hline 2343.997(16) & 42649.1 & $2343.997(19)$ & & 28 & & & & $5 \mathrm{~s} 6 \mathrm{p}$ & ${ }^{3} \mathrm{P}^{\circ}{ }_{1} \quad 5 \mathrm{~s} 18 \mathrm{~d}$ & & 3 & P38P & \\
\hline $2350.7432(22)$ & 42526.72 & $2350.7423(4)$ & 0.0009 & 2800 & $1.7 \mathrm{e}+7$ & $\mathrm{D}$ & & $5 \mathrm{~s} 5 \mathrm{p}$ & ${ }^{1} \mathrm{P}_{1}^{\circ}$ & & & $\mathrm{K} 01 \mathrm{c}$ & $\mathrm{J} 07$ \\
\hline $2355.67(9)(1)$ & 42437.8 & $2355.862(11)$ & -0.19 & 110 & & & & $5 p^{2}$ & $5 \mathrm{~s} 11 \mathrm{p}$ & ${ }^{1} \mathrm{P}_{1}^{\circ}$ & & B69 & \\
\hline 2356.108(17) & 42429.9 & $2356.107(19)$ & & 230 & & & & $5 \mathrm{~s} 6 \mathrm{p}$ & $5 \mathrm{~s} 17 \mathrm{~d}$ & ${ }^{1} \mathrm{D}_{2}$ & 3 & P38P & \\
\hline $2356.88(3)$ & 42416.0 & $2356.88(3)$ & & $150^{*}$ & & & & $5 s 6 p$ & $5 \mathrm{~s} 17 \mathrm{~d}$ & & & P38P & \\
\hline 2356.88(3) & 42416.0 & $2356.88(3)$ & & $150^{*}$ & & & & $5 \mathrm{~s} 6 \mathrm{p}$ & $5 \mathrm{~s} 17 \mathrm{~d}$ & & & P38P & \\
\hline $2362.863(17)$ & 42308.6 & $2362.853(14)$ & 0.010 & 37 & & & E2 & $5 s 6 p$ & $5 \mathrm{~s} 16 \mathrm{~d}$ & ${ }^{3} \mathrm{D}_{2}$ & & P38P & \\
\hline $2363.037(17)$ & 42305.5 & $2363.009(13)$ & 0.028 & 34 & & & & $5 \mathrm{~s} 6 \mathrm{p}$ & $5 \mathrm{~s} 16 \mathrm{~d}$ & ${ }^{3} \mathrm{D}_{1}$ & & P38P & \\
\hline $2364.6858699778(4)$ & 42275.995245348 & $2364.6858699778(4)$ & $0 \mathrm{e}-10$ & 220 & $5.1 \mathrm{e}+0$ & $\mathrm{~B}+$ & $\mathrm{HF}$ & $5 s^{2}$ & $5 \mathrm{~s} 5 \mathrm{p}$ & ${ }^{3} \mathrm{P}_{0}^{\circ}$ & & Z01 & B01 \\
\hline $2365.721(17)$ & 42257.5 & $2365.723(19)$ & & 54 & & & & $5 s 6 p$ & ${ }^{3} \mathrm{P}_{2}^{\circ} \quad 5 \mathrm{~s} 19 \mathrm{~d}$ & ${ }^{3} \mathrm{D}_{3}$ & & P38P & \\
\hline 2367.009(17) & 42234.5 & 2367.015(9) & -0.006 & 31 & $1.9 \mathrm{e}+5$ & $\mathrm{D}+$ & & $5 \mathrm{~s} 6 \mathrm{p}$ & $5 \mathrm{~s} 17 \mathrm{~s}$ & & & P38P & TW \\
\hline $2370.59(9)(2)$ & 42170.7 & $2370.459(5)$ & 0.13 & 580 & $1.5 \mathrm{e}+5$ & $\mathrm{E}$ & & $5 \mathrm{~s} 6 \mathrm{~s}$ & $5 \mathrm{~s} 8 \mathrm{p}$ & ${ }^{1} \mathrm{P}^{\circ}{ }_{1}$ & 1 & B69 & TW \\
\hline 2372.63(9)(2) & 42134.5 & $2372.370(7)$ & 0.26 & 43 & $8 \mathrm{e}+4$ & $\mathrm{E}$ & & $5 p^{2}$ & $5 \mathrm{~s} 10 \mathrm{f}$ & ${ }^{1} \mathrm{~F}_{3}^{\circ}$ & 1 & B69 & $\mathrm{TW}$ \\
\hline $2372.904(17)$ & 42129.6 & $2372.909(15)$ & -0.005 & 76 & & & & $5 s 6 p$ & $5 \mathrm{~s} 16 \mathrm{~d}$ & & & P38P & \\
\hline 2373.039(17) & 42127.2 & $2373.066(14)$ & -0.027 & 76 & & & & $5 s 6 p$ & ${ }^{3} \mathrm{P}_{1}^{\circ} \quad 5 \mathrm{~s} 16 \mathrm{~d}$ & ${ }^{3} \mathrm{D}_{1}$ & & P38P & \\
\hline 2373.73(9)(2) & 42114.9 & $2373.716(8)$ & 0.01 & 220 & & & & $5 p^{2}$ & $5 \mathrm{~s} 10 \mathrm{f}$ & ${ }^{3} \mathrm{~F}_{3}^{\circ}$ & & B69 & \\
\hline $2374.80(9)(2)$ & 42096.0 & $2374.658(12)$ & 0.14 & 100 & & & & $5 p^{2}$ & $5 \mathrm{~s} 10 \mathrm{p}$ & ${ }^{3} \mathrm{P}^{\circ}{ }_{1}$ & & B69 & \\
\hline $2375.960(17)$ & 42075.4 & $2375.956(13)$ & 0.004 & 130 & $2.3 \mathrm{e}+6$ & E & & $5 \mathrm{~s} 6 \mathrm{~s}$ & ${ }^{3} \mathrm{~S}_{1} \quad 5 \mathrm{~s} 8 \mathrm{p}$ & ${ }^{3} \mathrm{P}_{2}^{\circ}$ & & P38P & B99 \\
\hline $2376.655(17)$ & 42063.1 & $2376.652(17)$ & 0.003 & 100 & & & & $5 s 6 p$ & ${ }^{3} \mathrm{P}_{2}^{\circ} \quad 5 \mathrm{~s} 18 \mathrm{~d}$ & ${ }^{3} \mathrm{D}_{3}$ & & P38P & \\
\hline $2377.085(17)$ & 42055.5 & $2377.106(9)$ & -0.021 & 64 & $5.4 \mathrm{e}+5$ & $\mathrm{D}+$ & & $5 \mathrm{~s} 6 \mathrm{p}$ & ${ }^{3} \mathrm{P}_{1}^{\circ} \quad 5 \mathrm{~s} 17 \mathrm{~s}$ & ${ }^{3} \mathrm{~S}_{1}$ & & P38P & TW \\
\hline $2382.630(11)$ & 41957.63 & $2382.626(5)$ & 0.004 & 4800 & $4.6 \mathrm{e}+7$ & $\mathrm{C}+$ & & $5 \mathrm{~s} 5 \mathrm{~d}$ & ${ }^{1} \mathrm{D}_{2} \quad 5 \mathrm{~s} 6 \mathrm{f}$ & ${ }^{1} \mathrm{~F}_{3}^{\circ}$ & 2 & P38G4 & B99 \\
\hline $2383.76(9)(2)$ & 41937.7 & $2383.760(3)$ & 0.00 & 300 & $2.0 \mathrm{e}+6$ & $\mathrm{E}$ & & $5 \mathrm{~s} 6 \mathrm{~s}$ & ${ }^{3} \mathrm{~S}_{1} \quad 5 \mathrm{~s} 8 \mathrm{p}$ & ${ }^{3} \mathrm{P}_{1}^{\circ}$ & & B69 & B99 \\
\hline
\end{tabular}


Volume 118 (2013) http://dx.doi.org/10.6028/jres.118.004

Journal of Research of the National Institute of Standards and Technology

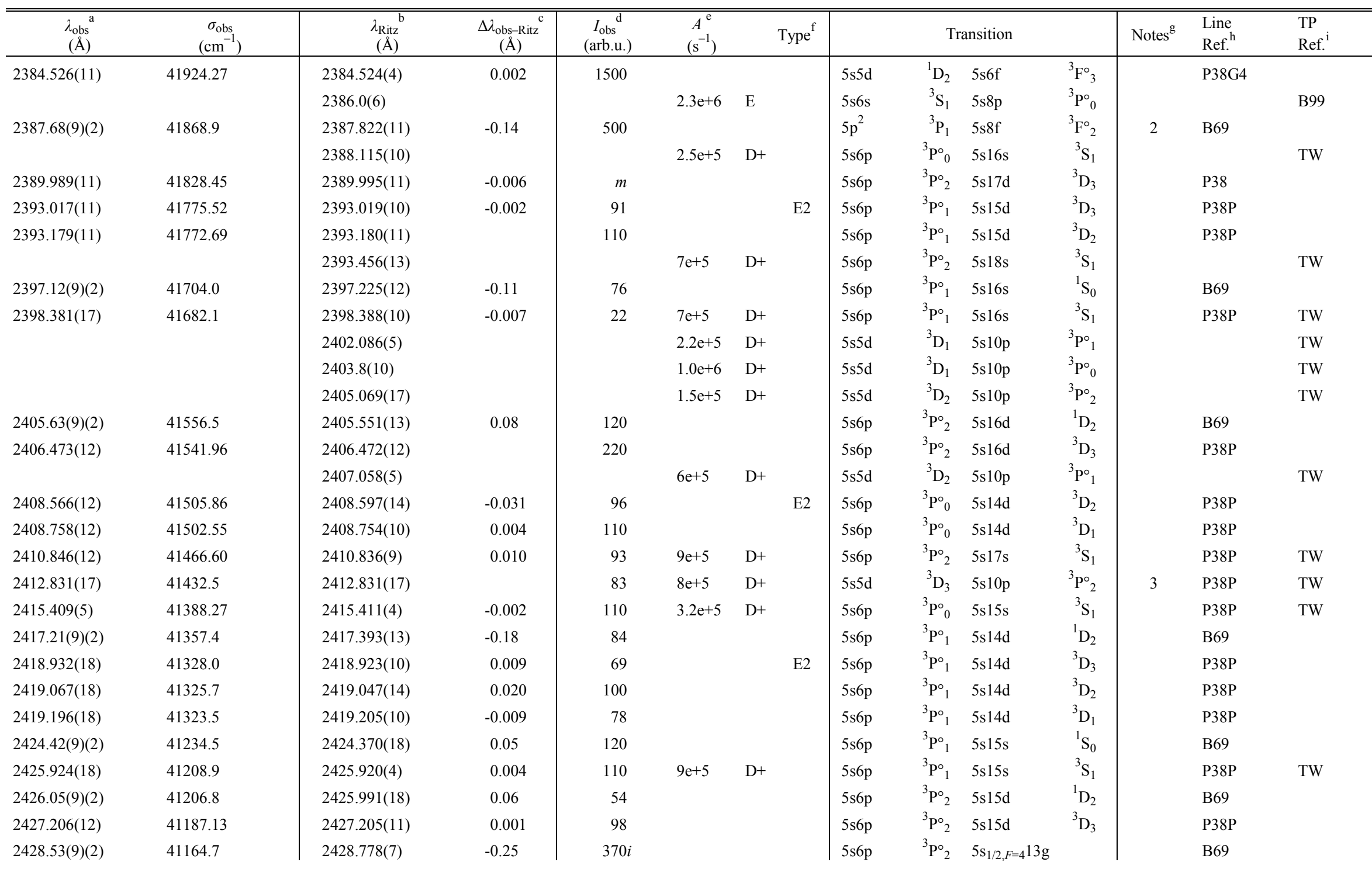


Volume 118 (2013) http://dx.doi.org/10.6028/jres.118.004

Journal of Research of the National Institute of Standards and Technology

\begin{tabular}{|c|c|c|c|c|c|c|c|c|c|c|c|c|c|}
\hline $\begin{array}{c}\lambda_{\text {obs }}^{\mathrm{a}} \\
(\AA) \\
\end{array}$ & $\begin{array}{c}\sigma_{\mathrm{obs}} \\
\left(\mathrm{cm}^{-1}\right)\end{array}$ & $\begin{array}{c}\lambda_{\text {Ritz }}{ }^{b} \\
(\AA)\end{array}$ & $\begin{array}{c}\Delta \lambda_{\text {obs-Ritz }}{ }^{c} \\
(\AA)\end{array}$ & $\begin{array}{c}\begin{array}{c}I_{\text {obs }}{ }^{2} \\
\text { (arb.u.) }\end{array} \\
\end{array}$ & $\begin{array}{c}A^{\mathrm{e}} \\
\left(\mathrm{s}^{-1}\right) \\
\end{array}$ & & Type $^{f}$ & & Transition & & Notes $^{\mathrm{g}}$ & $\begin{array}{l}\text { Line } \\
\text { Ref. }^{h}\end{array}$ & $\begin{array}{l}\text { TP } \\
\text { Ref. }\end{array}$ \\
\hline 2432.731(12) & 41093.59 & $\begin{array}{l}2432.729(10) \\
2433.946(8)\end{array}$ & 0.002 & 50 & $\begin{array}{l}1.2 \mathrm{e}+6 \\
7 \mathrm{e}+5\end{array}$ & $\begin{array}{l}\mathrm{D}^{+} \\
\mathrm{D}+\end{array}$ & & $\begin{array}{l}5 s 6 p \\
5 p^{2}\end{array}$ & $\begin{array}{ll}{ }^{3} \mathrm{P}_{2}^{\circ} & 5 \mathrm{~s} 16 \mathrm{~s} \\
{ }^{3} \mathrm{P}_{2} & 5 \mathrm{~s} 9 \mathrm{f}\end{array}$ & $\begin{array}{r}{ }^{3} \mathrm{~S}_{1} \\
{ }^{1} \mathrm{~F}^{\circ}{ }_{3}\end{array}$ & & P38P & $\begin{array}{l}\text { TW } \\
\text { TW }\end{array}$ \\
\hline 2442.471(12) & 40929.74 & $2442.480(11)$ & -0.009 & 67 & & & E2 & $5 s 6 p$ & ${ }^{3} \mathrm{P}_{0}^{\circ} \quad 5 \mathrm{~s} 13 \mathrm{~d}$ & ${ }^{3} \mathrm{D}_{2}$ & & P38P & \\
\hline $2442.634(12)$ & 40927.01 & $2442.634(12)$ & & 110 & & & & $5 \mathrm{~s} 6 \mathrm{p}$ & ${ }^{3} \mathrm{P}_{0}^{\circ} \quad 5 \mathrm{~s} 13 \mathrm{~d}$ & ${ }^{3} \mathrm{D}_{1}$ & & P38P & \\
\hline $2447.886(12)$ & 40839.21 & 2447.893(3) & -0.007 & 220 & $1.0 \mathrm{e}+7$ & $\mathrm{D}^{+}$ & & $5 \mathrm{~s} 5 \mathrm{~d}$ & ${ }^{3} \mathrm{D}_{1} \quad 5 \mathrm{~s} 7 \mathrm{f}$ & & & P38P & TW \\
\hline 2451.121(12) & 40785.30 & $2451.135(5)$ & -0.014 & 2000 & & & & $5 \mathrm{~s} 5 \mathrm{~d}$ & ${ }^{3} \mathrm{D}_{2} \quad 5 \mathrm{~s} 7 \mathrm{f}$ & ${ }^{1} \mathrm{~F}_{3}^{\circ}$ & & P38G4 & \\
\hline $2451.549(5)$ & 40778.19 & $2451.550(4)$ & -0.001 & 36 & $4.4 \mathrm{e}+5$ & $\mathrm{D}+$ & & $5 \mathrm{~s} 6 \mathrm{p}$ & ${ }^{3} \mathrm{P}_{0}^{\circ} \quad 5 \mathrm{~s} 14 \mathrm{~s}$ & & & P38P & TW \\
\hline $2452.910(12)$ & 40755.57 & $\begin{array}{l}2452.912(3) \\
2453.0571(23)\end{array}$ & -0.002 & 1400 & $\begin{array}{l}1.1 \mathrm{e}+7 \\
1.9 \mathrm{e}+6\end{array}$ & $\begin{array}{l}\mathrm{D}^{+} \\
\mathrm{D}+\end{array}$ & & $\begin{array}{l}5 \mathrm{~s} 5 \mathrm{~d} \\
5 \mathrm{~s} 5 \mathrm{~d}\end{array}$ & $\begin{array}{ll}{ }^{3} \mathrm{D}_{2} & 5 \mathrm{~s} 7 \mathrm{f} \\
{ }^{3} \mathrm{D}_{2} & 5 \mathrm{~s} 7 \mathrm{f}\end{array}$ & $\begin{array}{l}{ }^{3} \mathrm{~F}^{\circ}{ }_{3} \\
{ }^{3} \mathrm{~F}_{2}^{\circ}\end{array}$ & & P38G4 & $\begin{array}{l}\text { TW } \\
\text { TW }\end{array}$ \\
\hline $2453.229(12)$ & 40750.26 & $2453.227(11)$ & 0.002 & 89 & & & & $5 \mathrm{~s} 6 \mathrm{p}$ & ${ }^{3} \mathrm{P}^{\circ}{ }_{1} \quad 5 \mathrm{~s} 13 \mathrm{~d}$ & ${ }^{3} \mathrm{D}_{2}$ & & P38P & \\
\hline $2453.856(12)$ & 40739.85 & $2453.858(11)$ & -0.002 & 92 & & & & $5 s 6 p$ & ${ }^{3} \mathrm{P}_{2}^{\circ} \quad 5 \mathrm{~s} 14 \mathrm{~d}$ & ${ }^{3} \mathrm{D}_{3}$ & & P38P & \\
\hline $2460.188(18)$ & 40635.0 & $\begin{array}{l}2460.198(9) \\
2460.986(3)\end{array}$ & -0.010 & 2500 & $\begin{array}{l}1.2 \mathrm{e}+7 \\
1.3 \mathrm{e}+6\end{array}$ & $\begin{array}{l}\mathrm{D}^{+} \\
\mathrm{D}+\end{array}$ & & $\begin{array}{l}5 \mathrm{~s} 5 \mathrm{~d} \\
5 \mathrm{~s} 5 \mathrm{~d}\end{array}$ & $\begin{array}{ll}{ }^{3} \mathrm{D}_{3} & 5 \mathrm{~s} 7 \mathrm{f} \\
{ }^{3} \mathrm{D}_{3} & 5 \mathrm{~s} 7 \mathrm{f}\end{array}$ & $\begin{array}{l}{ }^{3} \mathrm{~F}_{4}^{\circ} \\
{ }^{3} \mathrm{~F}_{3}^{\circ}\end{array}$ & & P38G4 & $\begin{array}{l}\text { TW } \\
\text { TW }\end{array}$ \\
\hline 2461.070(12) & 40620.44 & $2461.060(4)$ & 0.010 & 33 & $1.5 \mathrm{e}+6$ & $\mathrm{D}+$ & & $5 \mathrm{~s} 6 \mathrm{p}$ & ${ }^{3} \mathrm{P}_{2}^{\circ} \quad 5 \mathrm{~s} 15 \mathrm{~s}$ & ${ }^{3} \mathrm{~S}_{1}$ & & P38P & TW \\
\hline $2462.378(12)$ & 40598.87 & $2462.377(4)$ & 0.001 & 17 & $1.2 \mathrm{e}+6$ & $\mathrm{D}+$ & & $5 \mathrm{~s} 6 \mathrm{p}$ & ${ }^{3} \mathrm{P}^{\circ}{ }_{1} \quad 5 \mathrm{~s} 14 \mathrm{~s}$ & ${ }^{3} \mathrm{~S}_{1}$ & & P38G4 & TW \\
\hline $2486.152(12)$ & 40210.66 & $2486.141(9)$ & 0.011 & 160 & $5 e+5$ & $\mathrm{D}$ & & $5 \mathrm{~s} 5 \mathrm{p}$ & ${ }^{1} \mathrm{P}_{1}^{\circ} \quad 5 \mathrm{p}^{2}$ & ${ }^{3} \mathrm{P}_{1}$ & & P38P & J07 \\
\hline $2488.618(5)$ & 40170.82 & $2488.619(5)$ & -0.001 & 120 & & & & $5 \mathrm{~s} 6 \mathrm{p}$ & ${ }^{3} \mathrm{P}_{0}^{\circ} \quad 5 \mathrm{~s} 12 \mathrm{~d}$ & ${ }^{3} \mathrm{D}_{1}$ & & P38P & \\
\hline 2488.953(12) & 40165.42 & $2488.953(12)$ & & 110 & & & & $5 \mathrm{~s} 6 \mathrm{p}$ & ${ }^{3} \mathrm{P}_{2}^{\circ} \quad 5 \mathrm{~s} 13 \mathrm{~d}$ & ${ }^{3} \mathrm{D}_{3}$ & & P38P & \\
\hline $2496.318(12)$ & 40046.92 & 2496.315(9) & 0.003 & 81 & & & & $5 \mathrm{~s} 6 \mathrm{p}$ & ${ }^{3} \mathrm{P}_{1}^{\circ} \quad 5 \mathrm{~s} 12 \mathrm{~d}$ & ${ }^{1} \mathrm{D}_{2}$ & & P38P & \\
\hline 2498.591(12) & 40010.49 & $2498.588(4)$ & 0.003 & 93 & $2.1 \mathrm{e}+6$ & $\mathrm{D}+$ & & $5 \mathrm{~s} 6 \mathrm{p}$ & ${ }^{3} \mathrm{P}_{2}^{\circ} \quad 5 \mathrm{~s} 14 \mathrm{~s}$ & ${ }^{3} \mathrm{~S}_{1}$ & & $\mathrm{P} 38 \mathrm{P}$ & TW \\
\hline 2499.353(12) & 39998.29 & $2499.354(12)$ & -0.001 & 32 & & & E2 & $5 s 6 p$ & ${ }^{3} \mathrm{P}^{\circ}{ }_{1} \quad 5 \mathrm{~s} 12 \mathrm{~d}$ & ${ }^{3} \mathrm{D}_{3}$ & & $\mathrm{P} 38 \mathrm{P}$ & \\
\hline 2499.600(12) & 39994.34 & $2499.603(10)$ & -0.003 & 110 & & & & $5 s 6 p$ & ${ }^{3} \mathrm{P}_{1}^{\circ} \quad 5 \mathrm{~s} 12 \mathrm{~d}$ & ${ }^{3} \mathrm{D}_{2}$ & & P38P & \\
\hline $2501.002(5)$ & 39971.93 & $2501.003(4)$ & -0.001 & 67 & $6 e+5$ & $\mathrm{D}^{+}$ & & $5 s 6 p$ & ${ }^{3} \mathrm{P}_{0}^{\circ} \quad 5 \mathrm{~s} 13 \mathrm{~s}$ & ${ }^{3} \mathrm{~S}_{1}$ & & P38P & TW \\
\hline 2508.157(19) & 39857.9 & $2508.159(19)$ & & 53 & & & & $5 \mathrm{~s} 6 \mathrm{p}$ & ${ }^{1} \mathrm{P}_{1}^{\circ} \quad 5 \mathrm{~s} 15 \mathrm{~d}$ & ${ }^{1} \mathrm{D}_{2}$ & & P38P & \\
\hline $2509.320(5)$ & 39839.44 & $2509.320(4)$ & 0.000 & 13 & & & & $5 s 6 p$ & ${ }^{3} \mathrm{P}^{\circ}{ }_{1} \quad 5 \mathrm{~s} 13 \mathrm{~s}$ & ${ }^{1} \mathrm{~S}_{0}$ & & $\mathrm{P} 38 \mathrm{P}$ & \\
\hline $2512.274(9)$ & 39792.60 & $2512.272(4)$ & 0.002 & 96 & $1.7 \mathrm{e}+6$ & $\mathrm{D}^{+}$ & & $5 s 6 p$ & ${ }^{3} \mathrm{P}^{\circ}{ }_{1} \quad 5 \mathrm{~s} 13 \mathrm{~s}$ & ${ }^{3} \mathrm{~S}_{1}$ & & P38P & TW \\
\hline $2514.082(13)$ & 39763.98 & $2514.082(13)$ & & 28 & $8 \mathrm{e}+5$ & $\mathrm{E}$ & & $5 \mathrm{~s} 6 \mathrm{p}$ & ${ }^{1} \mathrm{P}^{\circ}{ }_{1} \quad 5 \mathrm{~s} 16 \mathrm{~s}$ & ${ }^{1} \mathrm{~S}_{0}$ & 1 & P38P & TW \\
\hline $2536.6710(19)$ & 39409.9 & $2536.669(12)$ & 0.002 & 400 & & & & $5 \mathrm{~s} 6 \mathrm{p}$ & ${ }^{3} \mathrm{P}_{2}^{\circ} \quad 5 \mathrm{~s} 12 \mathrm{~d}$ & ${ }^{3} \mathrm{D}_{3}$ & & P38G2 & \\
\hline $2543.953(19)$ & 39297.1 & $2543.955(19)$ & & 100 & $9 e+5$ & E & & $5 \mathrm{~s} 6 \mathrm{p}$ & ${ }^{1} \mathrm{P}_{1}^{\circ} \quad 5 \mathrm{~s} 15 \mathrm{~s}$ & ${ }^{1} \mathrm{~S}_{0}$ & 1 & P38G2 & TW \\
\hline $2545.747(13)$ & 39269.41 & $2545.7185(10)$ & 0.028 & 490 & & & E2 & $5 \mathrm{~s} 5 \mathrm{p}$ & ${ }^{1} \mathrm{P}_{1}^{\circ} \quad 5 \mathrm{~s} 5 \mathrm{~d}$ & ${ }^{3} \mathrm{D}_{3}$ & & P38G2 & \\
\hline
\end{tabular}


Volume 118 (2013) http://dx.doi.org/10.6028/jres.118.004

Journal of Research of the National Institute of Standards and Technology

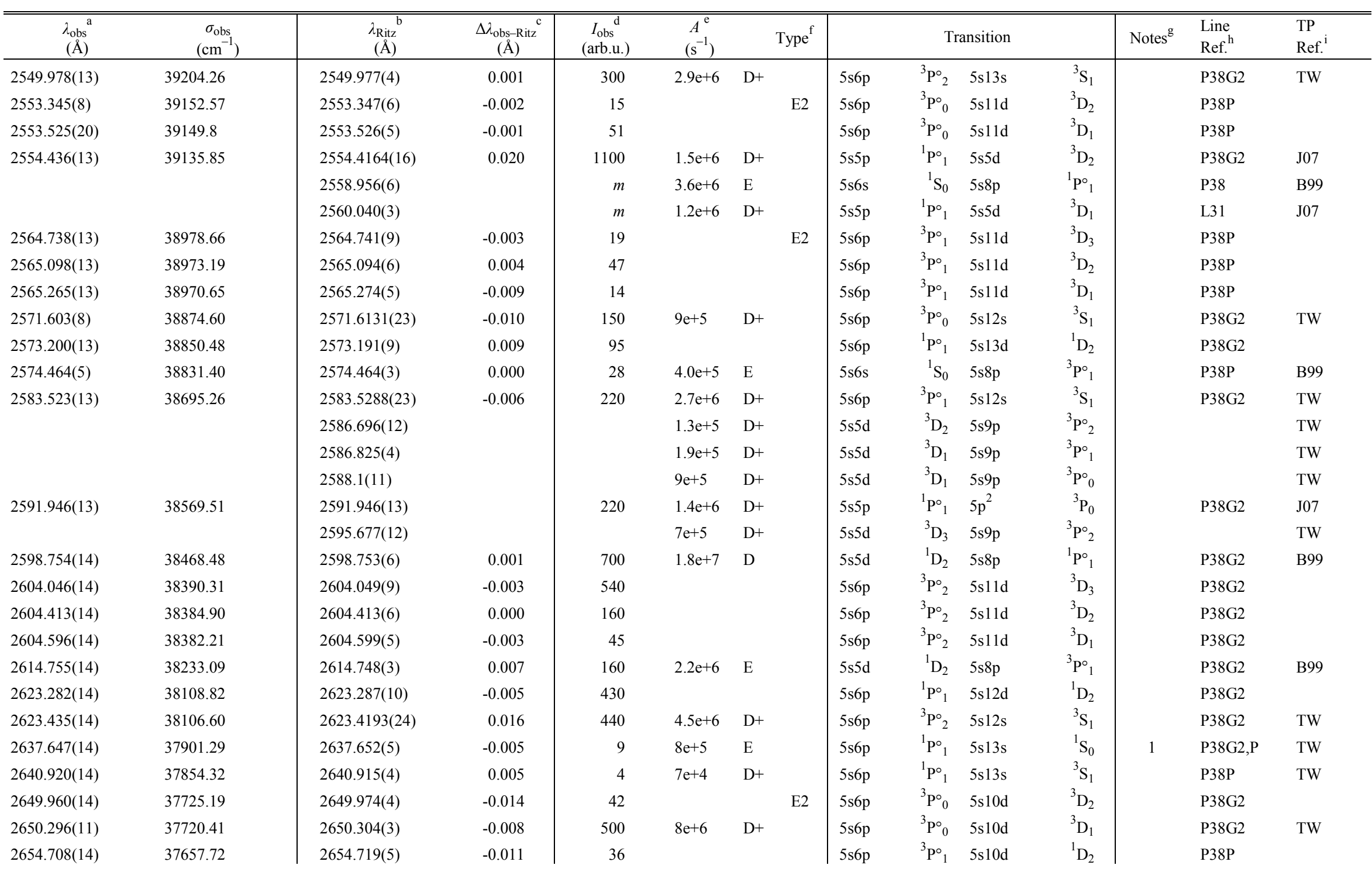


Volume 118 (2013) http://dx.doi.org/10.6028/jres.118.004

Journal of Research of the National Institute of Standards and Technology

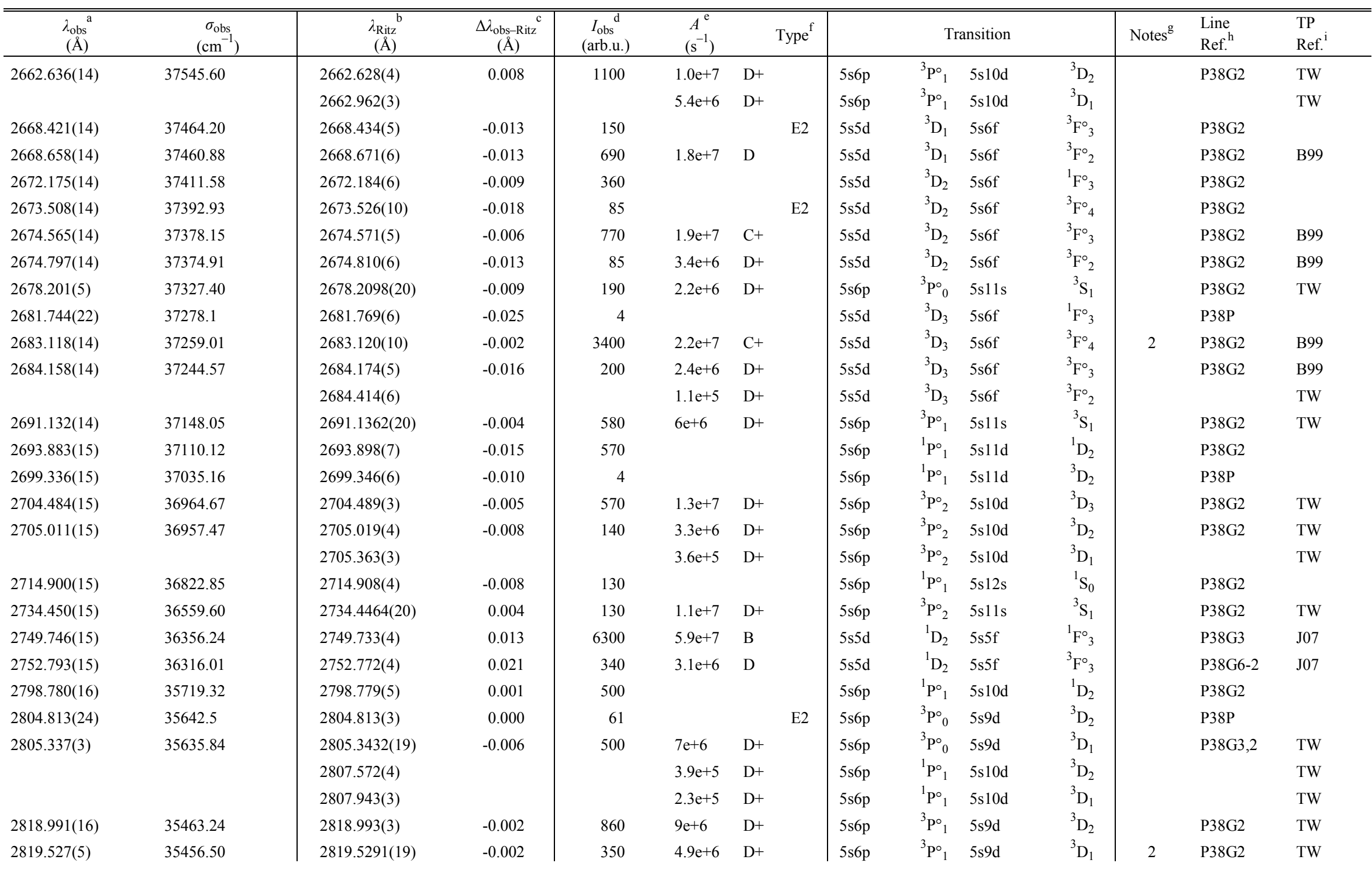


Volume 118 (2013) http://dx.doi.org/10.6028/jres.118.004

Journal of Research of the National Institute of Standards and Technology

\begin{tabular}{|c|c|c|c|c|c|c|c|c|c|c|c|c|}
\hline $\begin{array}{c}\lambda_{\text {obs }}{ }^{a} \\
(\AA)\end{array}$ & $\begin{array}{c}\sigma_{\mathrm{obs}} \\
\left(\mathrm{cm}^{-1}\right)\end{array}$ & $\begin{array}{c}\lambda_{\text {Ritz }} \text { b } \\
(\AA)\end{array}$ & $\begin{array}{c}\Delta \lambda_{\text {obs-Ritz }}{ }^{c} \\
(\AA)\end{array}$ & $\begin{array}{c}I_{\mathrm{obs}}^{\mathrm{d}} \\
\text { (arb.u.) }\end{array}$ & $\begin{array}{c}A^{\mathrm{e}} \\
\left(\mathrm{s}^{-1}\right)\end{array}$ & Type ${ }^{f}$ & \multicolumn{3}{|c|}{ Transition } & Notes $^{\mathrm{g}}$ & $\begin{array}{l}\text { Line } \\
\text { Ref. }^{h}\end{array}$ & $\begin{array}{l}\text { TP } \\
\text { Ref. }^{\text {i }}\end{array}$ \\
\hline $2831.539(16)$ & 35306.10 & $2831.544(3)$ & -0.005 & 330 & $5 e+6$ & $\mathrm{E}$ & $5 \mathrm{~s} 6 \mathrm{p}$ & $5 \mathrm{~s} 11 \mathrm{~s}$ & ${ }^{1} \mathrm{~S}_{0}$ & 1 & P38G3,2 & TW \\
\hline $2839.53(13)(5)$ & 35206.7 & $2839.2860(22)$ & 0.24 & 190 & $2.7 \mathrm{e}+5$ & $\mathrm{D}+$ & $5 \mathrm{~s} 6 \mathrm{p}$ & ${ }^{1} \mathrm{P}_{1}^{\circ} \quad 5 \mathrm{~s} 11 \mathrm{~s}$ & & & B69 & TW \\
\hline $2852.579(3)$ & 35045.70 & $2852.5857(17)$ & -0.007 & 190 & $1.6 \mathrm{e}+6$ & $\mathrm{D}+$ & $5 \mathrm{~s} 6 \mathrm{p}$ & $5 \mathrm{~s} 10 \mathrm{~s}$ & ${ }^{3} \mathrm{~S}_{1}$ & & P38G3,2 & TW \\
\hline 2865.682(16) & 34885.46 & $2865.684(12)$ & -0.002 & 450 & $1.2 \mathrm{e}+7$ & $\mathrm{D}+$ & $5 \mathrm{~s} 6 \mathrm{p}$ & ${ }^{3} \mathrm{P}_{2}^{\circ}$ & & 2 & P38G3,2 & TW \\
\hline \multirow[t]{2}{*}{$2866.543(16)$} & 34874.99 & $2866.552(3)$ & -0.009 & 380 & $2.9 \mathrm{e}+6$ & $\mathrm{D}+$ & $5 \mathrm{~s} 6 \mathrm{p}$ & $5 \mathrm{~s} 9 \mathrm{~d}$ & & & P38G3,2 & TW \\
\hline & & $2867.1065(20)$ & & & $3.3 \mathrm{e}+5$ & $\mathrm{D}^{+}$ & $5 \mathrm{~s} 6 \mathrm{p}$ & ${ }^{3} \mathrm{P}_{2}^{\circ} \quad 5 \mathrm{~s} 9 \mathrm{~d}$ & & & & TW \\
\hline 2867.251(3) & 34866.38 & $2867.2546(17)$ & -0.004 & 740 & $4.6 \mathrm{e}+6$ & $\mathrm{D}+$ & $5 \mathrm{~s} 6 \mathrm{p}$ & $5 \mathrm{~s} 10 \mathrm{~s}$ & ${ }^{3} \mathrm{~S}_{1}$ & & P38G3,2 & TW \\
\hline $2890.1708(3)$ & 34589.889 & $2890.17075(24)$ & 0.0000 & 10000 & $2.0 \mathrm{e}+7$ & $\mathrm{D}+$ & $5 \mathrm{~s} 5 \mathrm{p}$ & ${ }^{1} \mathrm{P}_{1}^{\circ}$ & ${ }^{1} \mathrm{D}_{2}$ & & $\mathrm{~K} 01 \mathrm{c}$ & A $86 \mathrm{c}$ \\
\hline \multirow[t]{2}{*}{$2916.466(17)$} & 34278.04 & $2916.4703(18)$ & -0.004 & 1100 & $8 \mathrm{e}+6$ & $\mathrm{D}^{+}$ & $5 s 6 p$ & $5 \mathrm{~s} 10 \mathrm{~s}$ & & & P38G3 & TW \\
\hline & & $2938.514(7)$ & & & $4.7 \mathrm{e}+5$ & $\mathrm{E}$ & $5 p^{2}$ & ${ }^{3} \mathrm{P}_{2} \quad 5 \mathrm{~s} 6 \mathrm{f}$ & & & & B99 \\
\hline \multirow[t]{5}{*}{ 2941.0373(10) } & 33991.669 & $2941.0376(8)$ & -0.0003 & 9600 & $3.4 \mathrm{e}+8$ & B & $5 \mathrm{~s} 5 \mathrm{p}$ & ${ }^{1} \mathrm{P}_{1}^{\circ} \quad 5 \mathrm{~s} 6 \mathrm{~s}$ & & & K01c & A86c \\
\hline & & $2955.558(20)$ & & & $1.5 \mathrm{e}+5$ & $\mathrm{D}+$ & $5 \mathrm{~s} 5 \mathrm{~d}$ & ${ }^{3} \mathrm{D}_{2} \quad 5 \mathrm{~s} 8 \mathrm{p}$ & & & & $\mathrm{TW}$ \\
\hline & & $2956.016(12)$ & & & $1.7 \mathrm{e}+7$ & $\mathrm{D}+$ & $5 p^{2}$ & ${ }^{1} \mathrm{D}_{2} \quad 5 \mathrm{~s} 10 \mathrm{f}$ & & & & TW \\
\hline & & $2960.089(5)$ & & & $2.1 \mathrm{e}+5$ & $\mathrm{D}+$ & $5 \mathrm{~s} 5 \mathrm{~d}$ & ${ }^{3} \mathrm{D}_{1} \quad 5 \mathrm{~s} 8 \mathrm{p}$ & & & & TW \\
\hline & & $2963.5(10)$ & & & $1.0 \mathrm{e}+6$ & $\mathrm{D}+$ & $5 \mathrm{~s} 5 \mathrm{~d}$ & ${ }^{3} \mathrm{D}_{1} \quad 5 \mathrm{~s} 8 \mathrm{p}$ & & & & B99 \\
\hline \multirow[t]{5}{*}{$2966.175(18)$} & 33703.61 & $2966.170(6)$ & 0.005 & 4300 & $1.9 \mathrm{e}+7$ & $\mathrm{D}^{+}$ & $5 \mathrm{~s} 6 \mathrm{p}$ & ${ }^{1} \mathrm{P}_{1}^{\circ} \quad 5 \mathrm{~s} 9 \mathrm{~d}$ & & & P38G4 & TW \\
\hline & & $2967.288(20)$ & & & $8 \mathrm{e}+5$ & $\mathrm{D}+$ & $5 \mathrm{~s} 5 \mathrm{~d}$ & ${ }^{3} \mathrm{D}_{3} \quad 5 \mathrm{~s} 8 \mathrm{p}$ & & & & B99 \\
\hline & & $2967.644(5)$ & & & $6 \mathrm{e}+5$ & $\mathrm{D}+$ & $5 \mathrm{~s} 5 \mathrm{~d}$ & ${ }^{3} \mathrm{D}_{2} \quad 5 \mathrm{~s} 8 \mathrm{p}$ & & & & B99 \\
\hline & & $2970.9507(7)$ & & & $2.7 \mathrm{e}+5$ & $\mathrm{D}$ & $5 \mathrm{~s} 6 \mathrm{~s}$ & ${ }^{3} \mathrm{~S}_{1} \quad 5 \mathrm{~s} 7 \mathrm{p}$ & & & & $\mathrm{J} 07$ \\
\hline & & $2981.979(3)$ & & & $2.4 \mathrm{e}+5$ & $\mathrm{D}+$ & $5 \mathrm{~s} 6 \mathrm{p}$ & ${ }^{1} \mathrm{P}_{1}^{\circ} \quad 5 \mathrm{~s} 9 \mathrm{~d}$ & & & & TW \\
\hline 2999.4092(4) & 33330.182 & 2999.4090(4) & 0.0002 & 1400 & $2.1 \mathrm{e}+6$ & $\mathrm{D}$ & $5 \mathrm{~s} 6 \mathrm{~s}$ & $5 \mathrm{~s} 7 \mathrm{p}$ & ${ }^{3} \mathrm{P}_{2}^{\circ}$ & & $\mathrm{K} 01 \mathrm{c}$ & $\mathrm{J} 07$ \\
\hline $3022.403(5)$ & 33076.62 & $3022.402(3)$ & 0.001 & 170 & $1.2 \mathrm{e}+7$ & E & $5 s 6 p$ & $5 \mathrm{~s} 10 \mathrm{~s}$ & & 1 & P38G2 & TW \\
\hline $3022.904(18)$ & 33071.14 & $3022.9163(9)$ & -0.012 & 84 & $8 \mathrm{e}+5$ & $\mathrm{D}$ & $5 \mathrm{~s} 6 \mathrm{~s}$ & $5 \mathrm{~s} 7 \mathrm{p}$ & ${ }^{3} \mathrm{P}_{1}^{\circ}$ & & P38G2 & J07 \\
\hline $3028.595(6)$ & 33009.00 & $3028.5958(19)$ & -0.001 & 67 & $3.1 \mathrm{e}+6$ & $\mathrm{D}+$ & $5 \mathrm{~s} 6 \mathrm{~s}$ & $5 \mathrm{~s} 7 \mathrm{p}$ & ${ }^{3} \mathrm{P}_{0}^{\circ}$ & & P38G2 & B99 \\
\hline $3052.228(19)$ & 32753.43 & $3052.226(12)$ & 0.002 & 240 & $7.2 \mathrm{e}+7$ & $\mathrm{C}$ & $5 p^{2}$ & ${ }^{1} \mathrm{D}_{2} \quad 5 \mathrm{~s} 9 \mathrm{f}$ & & 2 & P38G3,2 & TW \\
\hline $3069.757(19)$ & 32566.40 & $3069.760(4)$ & -0.003 & 170 & $1.2 \mathrm{e}+6$ & $\mathrm{D}+$ & $5 \mathrm{~s} 6 \mathrm{p}$ & ${ }^{3} \mathrm{P}_{1}^{\circ} \quad 5 \mathrm{~s} 8 \mathrm{~d}$ & ${ }^{1} \mathrm{D}_{2}$ & & P38P & TW \\
\hline $3083.663(4)$ & 32419.55 & $3083.6656(24)$ & -0.003 & 1000 & $1.2 \mathrm{e}+7$ & $\mathrm{D}+$ & $5 \mathrm{~s} 6 \mathrm{p}$ & $5 \mathrm{~s} 8 \mathrm{~d}$ & ${ }^{3} \mathrm{D}_{1}$ & & P38G3 & $\mathrm{TW}$ \\
\hline 3099.809(19) & 32250.69 & $3099.813(3)$ & -0.004 & 3800 & $1.6 \mathrm{e}+7$ & $\mathrm{D}+$ & $5 \mathrm{~s} 6 \mathrm{p}$ & $5 \mathrm{~s} 8 \mathrm{~d}$ & ${ }^{3} \mathrm{D}_{2}$ & 2 & P38G4 & TW \\
\hline $3100.814(6)$ & 32240.24 & $3100.8142(24)$ & 0.000 & 700 & $9 e+6$ & $\mathrm{D}+$ & $5 \mathrm{~s} 6 \mathrm{p}$ & $5 \mathrm{~s} 8 \mathrm{~d}$ & ${ }^{3} \mathrm{D}_{1}$ & & P38G2 & TW \\
\hline $3138.212(20)$ & 31856.05 & $3138.217(6)$ & -0.005 & 51 & & E2 & $5 \mathrm{~s} 5 \mathrm{~d}$ & $5 \mathrm{~s} 5 \mathrm{f}$ & ${ }^{3} \mathrm{~F}_{3}^{\circ}$ & & P38G4,2 & \\
\hline
\end{tabular}


Volume 118 (2013) http://dx.doi.org/10.6028/jres.118.004

Journal of Research of the National Institute of Standards and Technology

\begin{tabular}{|c|c|c|c|c|c|c|c|c|c|c|c|c|}
\hline $\begin{array}{c}\lambda_{\text {obs }}{ }^{\mathrm{a}} \\
(\AA)\end{array}$ & $\begin{array}{c}\sigma_{\mathrm{obs}} \\
\left(\mathrm{cm}^{-1}\right)\end{array}$ & $\begin{array}{c}\lambda_{\text {Ritz }}{ }^{b} \\
(\AA)\end{array}$ & $\begin{array}{c}\Delta \lambda_{\text {obs-Ritz }}{ }^{c} \\
(\AA)\end{array}$ & $\begin{array}{c}I_{\text {obs }}^{\mathrm{d}} \\
\text { (arb.u.) }\end{array}$ & $\begin{array}{c}A^{\mathrm{e}} \\
\left(\mathrm{s}^{-1}\right) \\
\end{array}$ & Type ${ }^{f}$ & \multicolumn{3}{|c|}{ Transition } & Notes $^{\mathrm{g}}$ & $\begin{array}{l}\text { Line } \\
\text { Ref. }^{h}\end{array}$ & $\begin{array}{l}\text { TP } \\
\text { Ref. }^{\text {i }}\end{array}$ \\
\hline $3138.610(5)$ & 31852.01 & $3138.609(4)$ & 0.001 & 1300 & $3.5 \mathrm{e}+7$ & $\mathrm{C}$ & $5 \mathrm{~s} 5 \mathrm{~d}$ & $5 \mathrm{~s} 5 \mathrm{f}$ & ${ }^{3} \mathrm{~F}_{2}^{\circ}$ & & K01c & $\mathrm{J} 07$ \\
\hline $3142.733(15)$ & 31810.22 & $3142.739(5)$ & -0.006 & 560 & $2.0 \mathrm{e}+6$ & $\mathrm{D}$ & $5 \mathrm{~s} 5 \mathrm{~d}$ & ${ }^{3} \mathrm{D}_{2} \quad 5 \mathrm{~s} 5 \mathrm{f}$ & & & P38G3,2 & J07 \\
\hline $3143.862(20)$ & 31798.80 & $3143.862(14)$ & 0.000 & 69 & & & $5 p^{2}$ & ${ }^{1} \mathrm{D}_{2} \quad 5 \mathrm{~s} 11 \mathrm{p}$ & & & P38G2 & \\
\hline $3145.137(20)$ & 31785.91 & $3145.110(4)$ & 0.027 & 66 & & E2 & $5 \mathrm{~s} 5 \mathrm{~d}$ & $5 \mathrm{~s} 5 \mathrm{f}$ & & & P38G2 & \\
\hline $3146.712(20)$ & 31770.00 & $3146.709(6)$ & 0.003 & 1600 & $3.5 \mathrm{e}+7$ & B & $5 \mathrm{~s} 5 \mathrm{~d}$ & ${ }^{3} \mathrm{D}_{2} \quad 5 \mathrm{~s} 5 \mathrm{f}$ & & & P38G3,2 & J07 \\
\hline $3147.099(10)$ & 31766.09 & $3147.103(4)$ & -0.004 & 210 & $6 e+6$ & D & $5 \mathrm{~s} 5 \mathrm{~d}$ & ${ }^{3} \mathrm{D}_{2} \quad 5 \mathrm{~s} 5 \mathrm{f}$ & & & P38G2 & J07 \\
\hline $3155.769(20)$ & 31678.83 & $3155.780(5)$ & -0.011 & 650 & $2.0 \mathrm{e}+7$ & $\mathrm{D}^{+}$ & $5 \mathrm{~s} 6 \mathrm{p}$ & $5 \mathrm{~s} 8 \mathrm{~d}$ & & & P38G2 & $\mathrm{TW}$ \\
\hline & & $3156.005(5)$ & & & $2.6 \mathrm{e}+5$ & $\mathrm{D}$ & $5 \mathrm{~s} 5 \mathrm{~d}$ & ${ }^{3} \mathrm{D}_{3} \quad 5 \mathrm{~s} 5 \mathrm{f}$ & & & & J07 \\
\hline $3157.411(20)$ & 31662.35 & $3157.416(3)$ & -0.005 & 120 & $5.1 \mathrm{e}+6$ & $\mathrm{D}+$ & $5 s 6 p$ & ${ }^{3} \mathrm{P}_{2}^{\circ} \quad 5 \mathrm{~s} 8 \mathrm{~d}$ & & & P38G2 & TW \\
\hline $3158.396(3)$ & 31652.48 & $3158.396(3)$ & 0.000 & 1800 & $4.2 \mathrm{e}+7$ & B & $5 \mathrm{~s} 5 \mathrm{~d}$ & ${ }^{3} \mathrm{D}_{3} \quad 5 \mathrm{~s} 5 \mathrm{f}$ & & & $\mathrm{K} 01 \mathrm{c}$ & J07 \\
\hline & & $3158.454(3)$ & & & $5.7 \mathrm{e}+5$ & $\mathrm{D}+$ & $5 s 6 p$ & ${ }^{3} \mathrm{P}_{2}^{\circ} \quad 5 \mathrm{~s} 8 \mathrm{~d}$ & & & & TW \\
\hline $3159.997(20)$ & 31636.44 & $3160.009(5)$ & -0.012 & 100 & $4.2 \mathrm{e}+6$ & $\mathrm{D}$ & $5 \mathrm{~s} 5 \mathrm{~d}$ & ${ }^{3} \mathrm{D}_{3} \quad 5 \mathrm{~s} 5 \mathrm{f}$ & ${ }^{3} \mathrm{~F}_{3}^{\circ}$ & & P38G2 & J07 \\
\hline & & $3160.406(4)$ & & & $1.7 \mathrm{e}+5$ & $\mathrm{D}$ & $5 \mathrm{~s} 5 \mathrm{~d}$ & ${ }^{3} \mathrm{D}_{3} \quad 5 \mathrm{~s} 5 \mathrm{f}$ & ${ }^{3} \mathrm{~F}_{2}^{\circ}$ & & & J07 \\
\hline $3176.272(4)$ & 31474.34 & $3176.2717(21)$ & 0.000 & 1400 & $2.6 \mathrm{e}+6$ & $\mathrm{D}+$ & $5 \mathrm{~s} 6 \mathrm{p}$ & ${ }^{3} \mathrm{P}_{0}^{\circ} \quad 5 \mathrm{~s} 9 \mathrm{~s}$ & ${ }^{3} \mathrm{~S}_{1}$ & & P38G3 & TW \\
\hline $3194.470(4)$ & 31295.05 & $3194.4688(21)$ & 0.001 & 1400 & $7 \mathrm{e}+6$ & $\mathrm{D}^{+}$ & $5 \mathrm{~s} 6 \mathrm{p}$ & ${ }^{3} \mathrm{P}_{1}^{\circ} \quad 5 \mathrm{~s} 9 \mathrm{~s}$ & ${ }^{3} \mathrm{~S}_{1}$ & & P38G3,2 & TW \\
\hline $3198.101(20)$ & 31259.52 & $3198.100(11)$ & 0.001 & 1300 & & & $5 p^{2}$ & ${ }^{1} \mathrm{D}_{2} \quad 5 \mathrm{~s} 8 \mathrm{f}$ & ${ }^{1} \mathrm{~F}_{3}^{\circ}$ & & P38G3 & \\
\hline $3200.851(20)$ & 31232.67 & $3200.850(10)$ & 0.001 & 96 & & & $5 p^{2}$ & ${ }^{1} \mathrm{D}_{2} \quad 5 \mathrm{~s} 8 \mathrm{f}$ & ${ }^{3} \mathrm{~F}_{3}^{\circ}$ & & $\mathrm{P} 38 \mathrm{G} 2$ & \\
\hline $3236.8463(22)$ & 30885.355 & $3236.8482(9)$ & -0.0019 & 450 & $9 e+5$ & $\mathrm{D}$ & $5 \mathrm{~s} 5 \mathrm{p}$ & ${ }^{1} \mathrm{P}_{1}^{\circ} \quad 5 \mathrm{~s} 6 \mathrm{~s}$ & ${ }^{3} \mathrm{~S}_{1}$ & & $\mathrm{~K} 01 \mathrm{c}$ & J07 \\
\hline & & $3255.6774(22)$ & & $m$ & $1.2 \mathrm{e}+7$ & $\mathrm{D}^{+}$ & $5 s 6 p$ & ${ }^{3} \mathrm{P}_{2}^{\circ} \quad 5 \mathrm{~s} 9 \mathrm{~s}$ & & & P38 & TW \\
\hline $3264.033(5)$ & 30628.11 & $3264.030(4)$ & 0.003 & 1700 & $2.6 \mathrm{e}+7$ & $\mathrm{D}^{+}$ & $5 \mathrm{~s} 6 \mathrm{p}$ & $5 \mathrm{~s} 8 \mathrm{~d}$ & ${ }^{1} \mathrm{D}_{2}$ & 2 & P38G4 & TW \\
\hline $3273.13(9)(14)$ & 30543.0 & $3273.1178(10)$ & 0.01 & 170 & $1.6 \mathrm{e}+6$ & $\mathrm{E}$ & $5 \mathrm{~s} 6 \mathrm{~s}$ & ${ }^{1} \mathrm{~S}_{0} \quad 5 \mathrm{~s} 7 \mathrm{p}$ & ${ }^{1} \mathrm{P}_{1}^{\circ}$ & & L31 & B99 \\
\hline $3298.019(5)$ & 30312.50 & $3298.028(3)$ & -0.009 & 130 & $4.3 \mathrm{e}+5$ & $\mathrm{D}^{+}$ & $5 \mathrm{~s} 6 \mathrm{p}$ & ${ }^{1} \mathrm{P}_{1}^{\circ} \quad 5 \mathrm{~s} 8 \mathrm{~d}$ & ${ }^{3} \mathrm{D}_{2}$ & & P38G2 & TW \\
\hline & & $3299.161(3)$ & & & $3.4 \mathrm{e}+5$ & $\mathrm{D}+$ & $5 s 6 p$ & ${ }^{\mathrm{l}} \mathrm{P}^{\circ}{ }_{1} \quad 5 \mathrm{~s} 8 \mathrm{~d}$ & ${ }^{3} \mathrm{D}_{1}$ & & & TW \\
\hline $3336.3042(23)$ & 29964.669 & $3336.3032(12)$ & 0.0010 & 66 & $2.5 \mathrm{e}+5$ & $\mathrm{E}$ & $5 \mathrm{~s} 6 \mathrm{~s}$ & $5 \mathrm{~s} 7 \mathrm{p}$ & ${ }^{3} \mathrm{P}_{1}^{\circ}$ & 1 & K01c & $\mathrm{TW}$ \\
\hline $3338.5087(3)$ & 29944.883 & $3338.5087(3)$ & 0.0000 & 2300 & $1.2 \mathrm{e}+7$ & $\mathrm{D}$ & $5 \mathrm{~s} 5 \mathrm{~d}$ & $5 \mathrm{~s} 7 \mathrm{p}$ & ${ }^{1} \mathrm{P}_{1}^{\circ}$ & 2 & K01c & J07 \\
\hline $3345.963(9)$ & 29878.17 & $3345.962(9)$ & 0.001 & 520 & $2.2 \mathrm{e}+7$ & E & $5 p^{2}$ & ${ }^{1} \mathrm{D}_{2} \quad 5 \mathrm{~s} 10 \mathrm{p}$ & ${ }^{1} \mathrm{P}^{\circ}{ }_{1}$ & 1 & P38G3 & TW \\
\hline $3352.308(9)$ & 29821.62 & $3352.308(9)$ & 0.000 & 350 & $3.2 \mathrm{e}+6$ & E & $5 p^{2}$ & $5 \mathrm{~s} 10 \mathrm{p}$ & ${ }^{3} \mathrm{P}_{1}^{\circ}$ & 1 & P38G3 & $\mathrm{TW}$ \\
\hline $3376.593(5)$ & 29607.15 & $3376.601(3)$ & -0.008 & 310 & $2.1 \mathrm{e}+7$ & E & $5 \mathrm{~s} 6 \mathrm{p}$ & $5 \mathrm{~s} 9 \mathrm{~s}$ & ${ }^{1} \mathrm{~S}_{0}$ & 1,2 & P38G3 & $\mathrm{TW}$ \\
\hline $3404.2701(12)$ & 29366.446 & $3404.2691(9)$ & 0.0010 & 600 & $6 e+5$ & $\mathrm{D}$ & $5 \mathrm{~s} 5 \mathrm{~d}$ & ${ }^{1} \mathrm{D}_{2} \quad 5 \mathrm{~s} 7 \mathrm{p}$ & ${ }^{3} \mathrm{P}_{1}^{\circ}$ & & $\mathrm{K} 01 \mathrm{c}$ & J07 \\
\hline & & $3405.3848(25)$ & & & $2.8 \mathrm{e}+5$ & $\mathrm{D}+$ & $5 s 6 p$ & $5 \mathrm{~s} 9 \mathrm{~s}$ & ${ }^{3} \mathrm{~S}_{1}$ & & & TW \\
\hline
\end{tabular}


Volume 118 (2013) http://dx.doi.org/10.6028/jres.118.004

Journal of Research of the National Institute of Standards and Technology

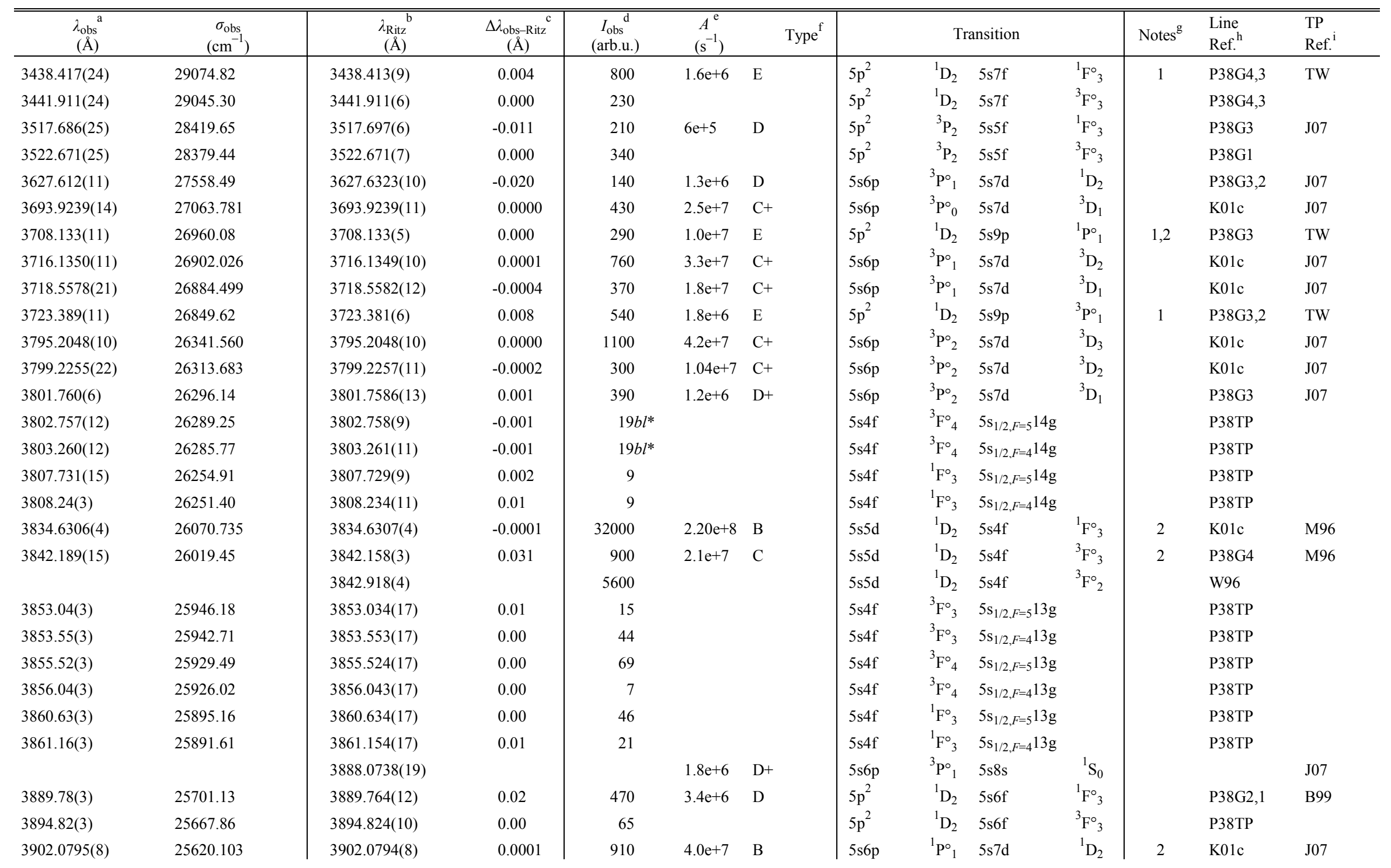


Volume 118 (2013) http://dx.doi.org/10.6028/jres.118.004

Journal of Research of the National Institute of Standards and Technology

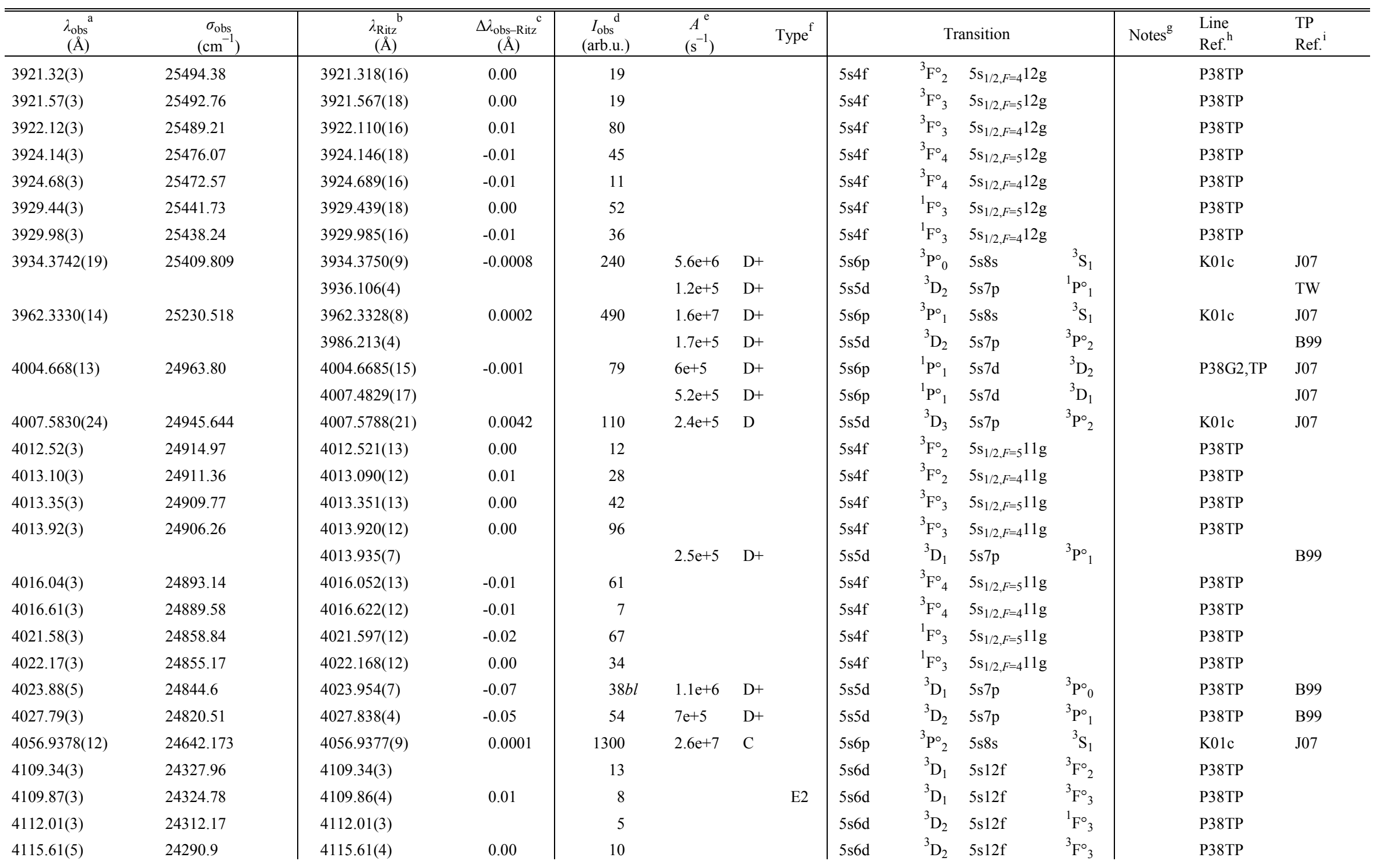


Volume 118 (2013) http://dx.doi.org/10.6028/jres.118.004

Journal of Research of the National Institute of Standards and Technology

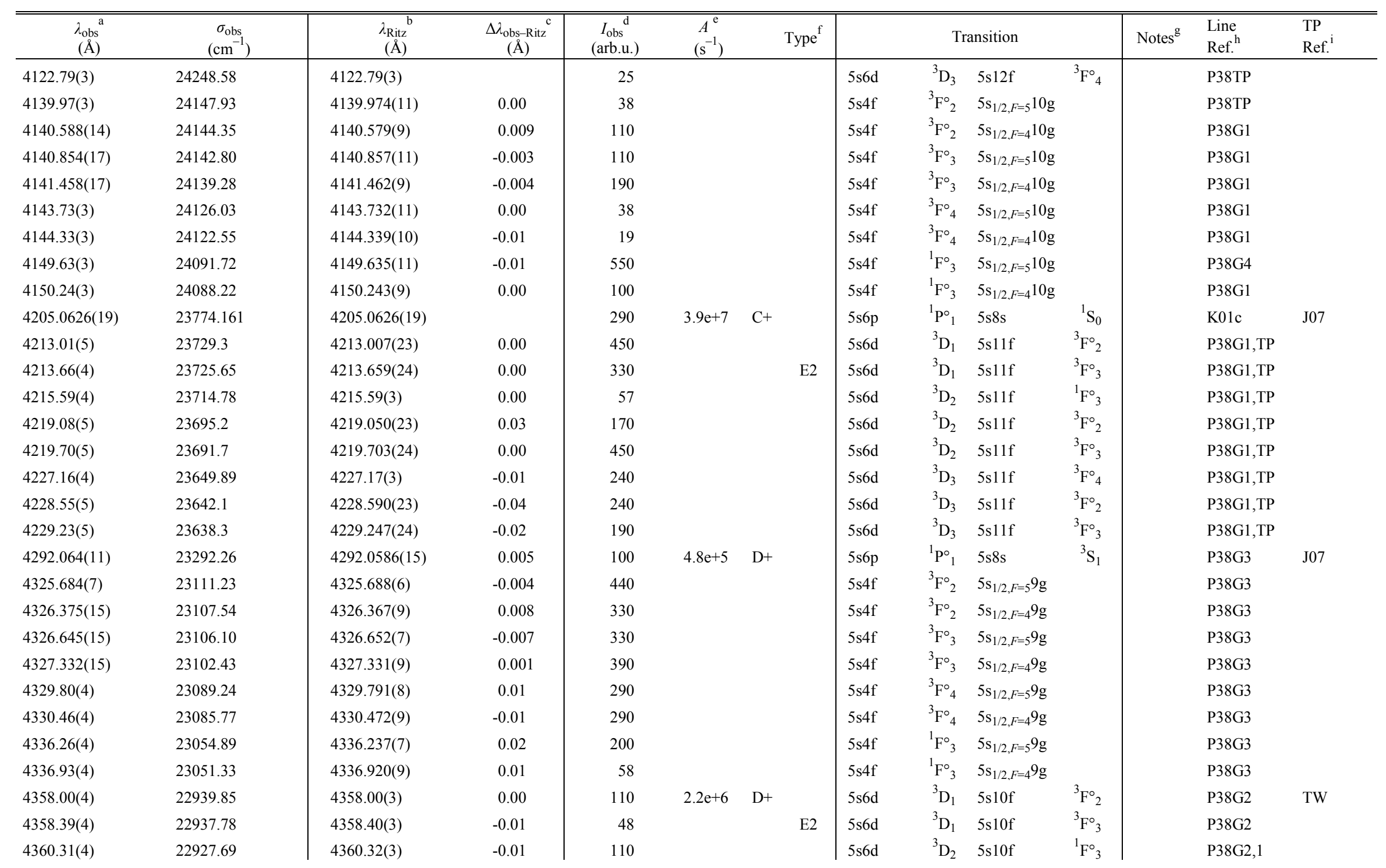


Volume 118 (2013) http://dx.doi.org/10.6028/jres.118.004

Journal of Research of the National Institute of Standards and Technology

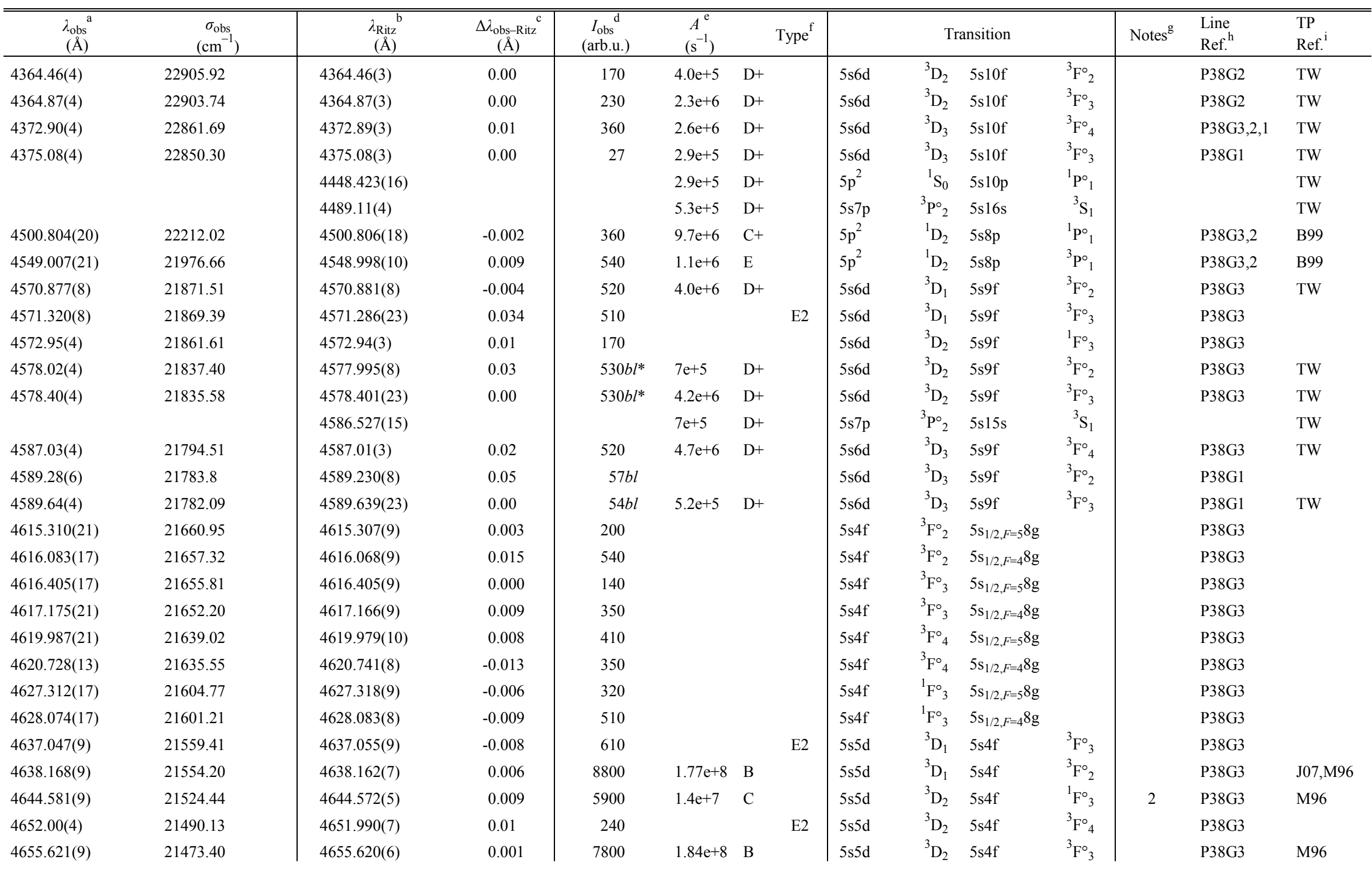


Volume 118 (2013) http://dx.doi.org/10.6028/jres.118.004

Journal of Research of the National Institute of Standards and Technology

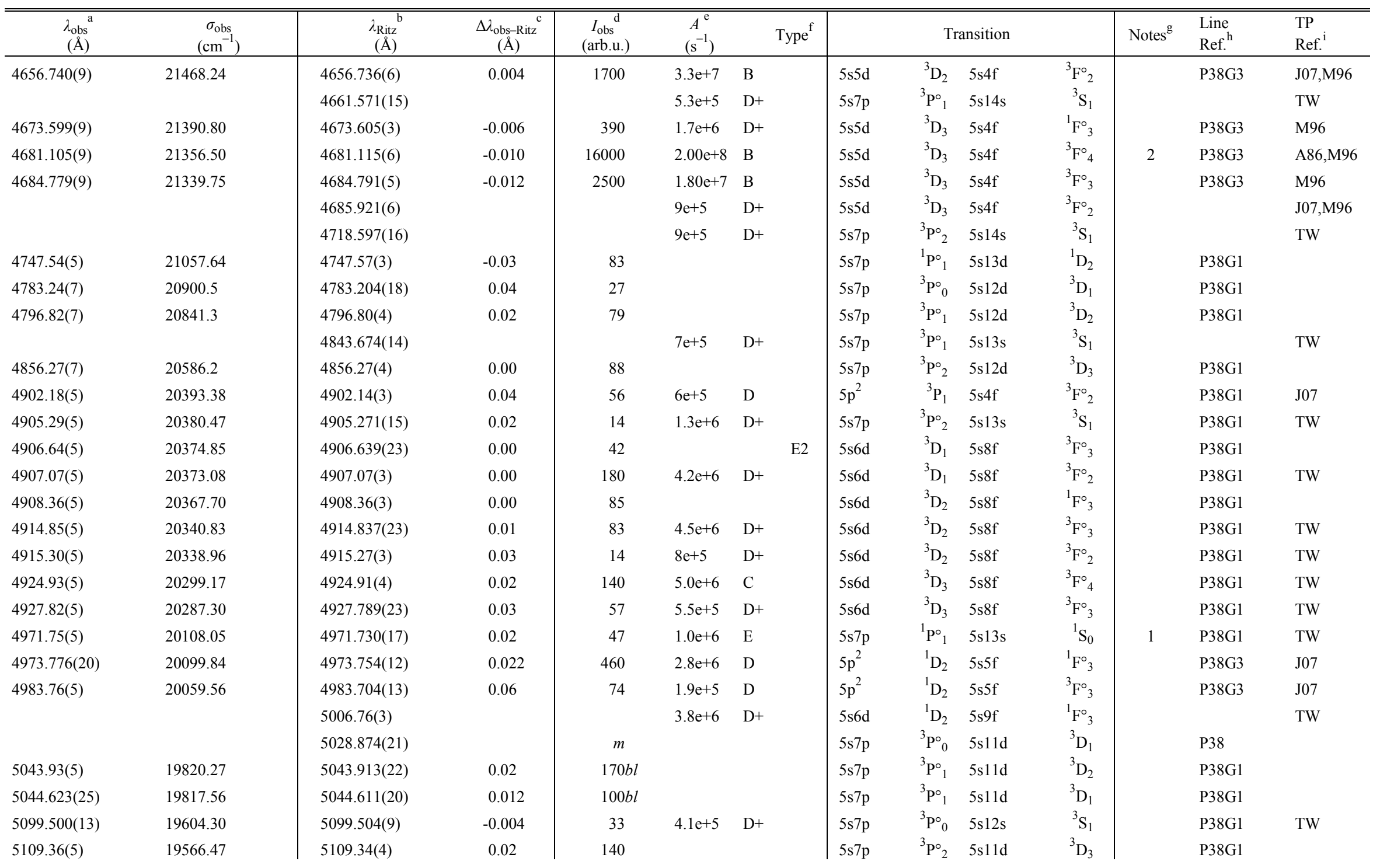


Volume 118 (2013) http://dx.doi.org/10.6028/jres.118.004

Journal of Research of the National Institute of Standards and Technology

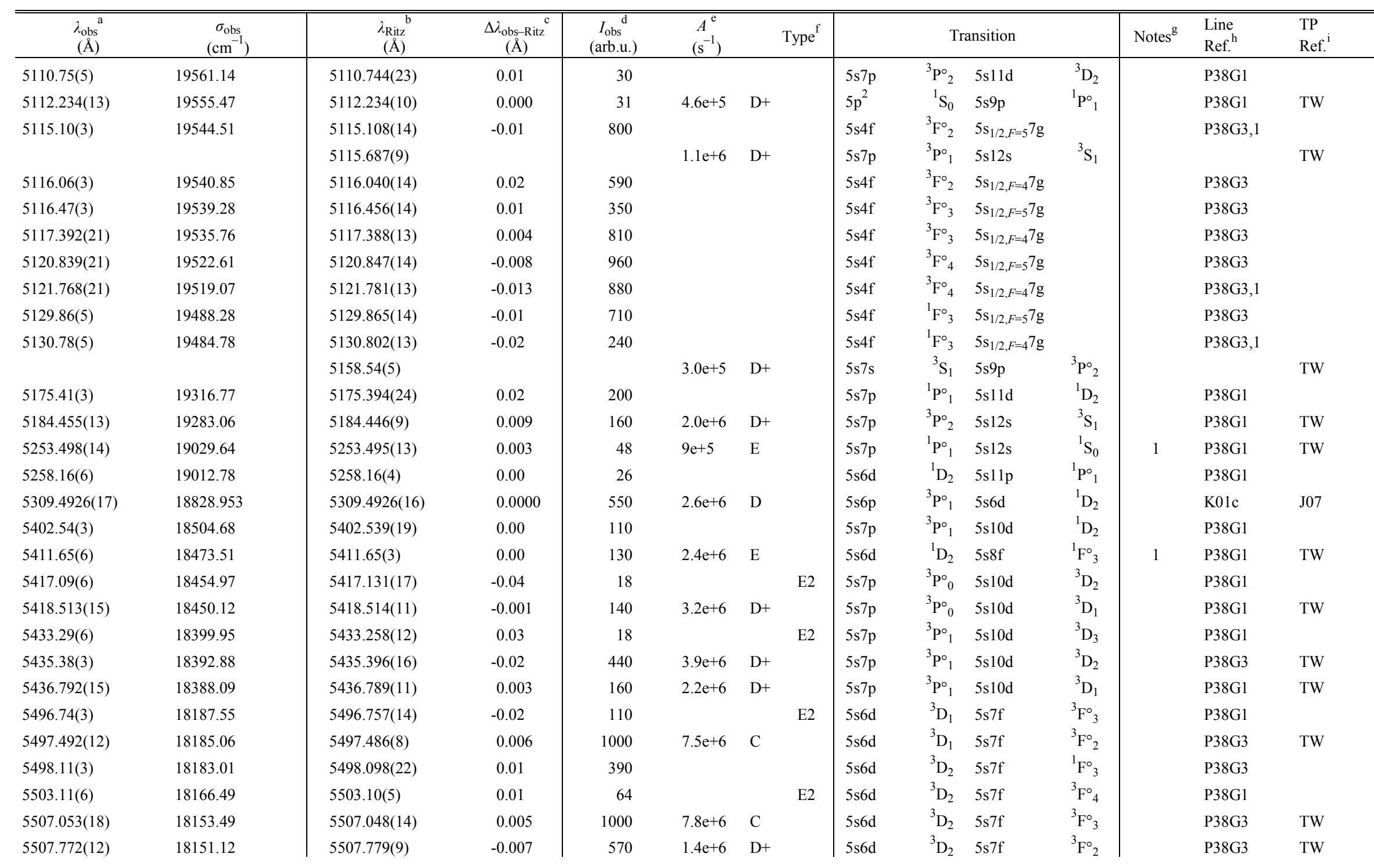


Volume 118 (2013) http://dx.doi.org/10.6028/jres.118.004

Journal of Research of the National Institute of Standards and Technology

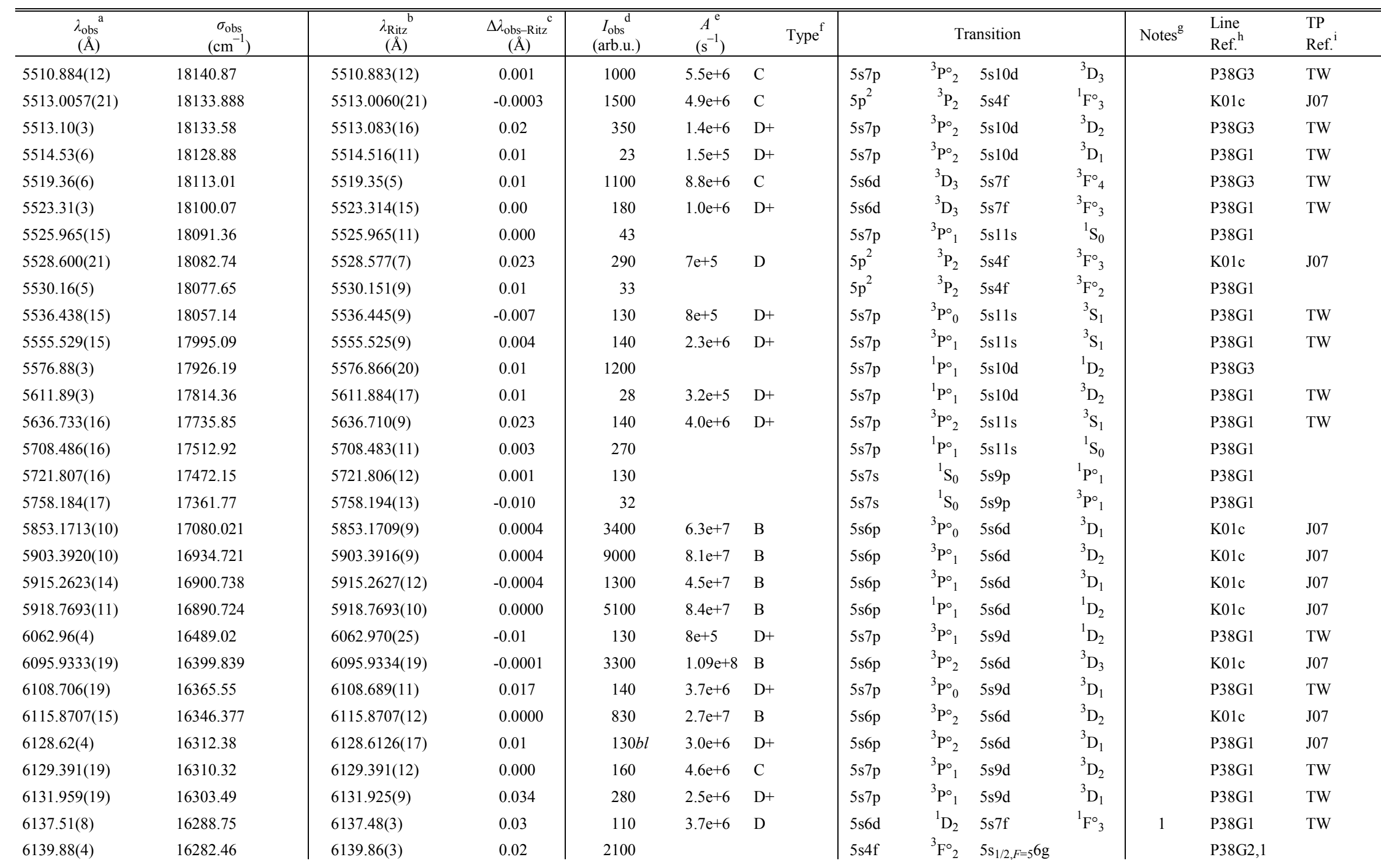


Volume 118 (2013) http://dx.doi.org/10.6028/jres.118.004

Journal of Research of the National Institute of Standards and Technology

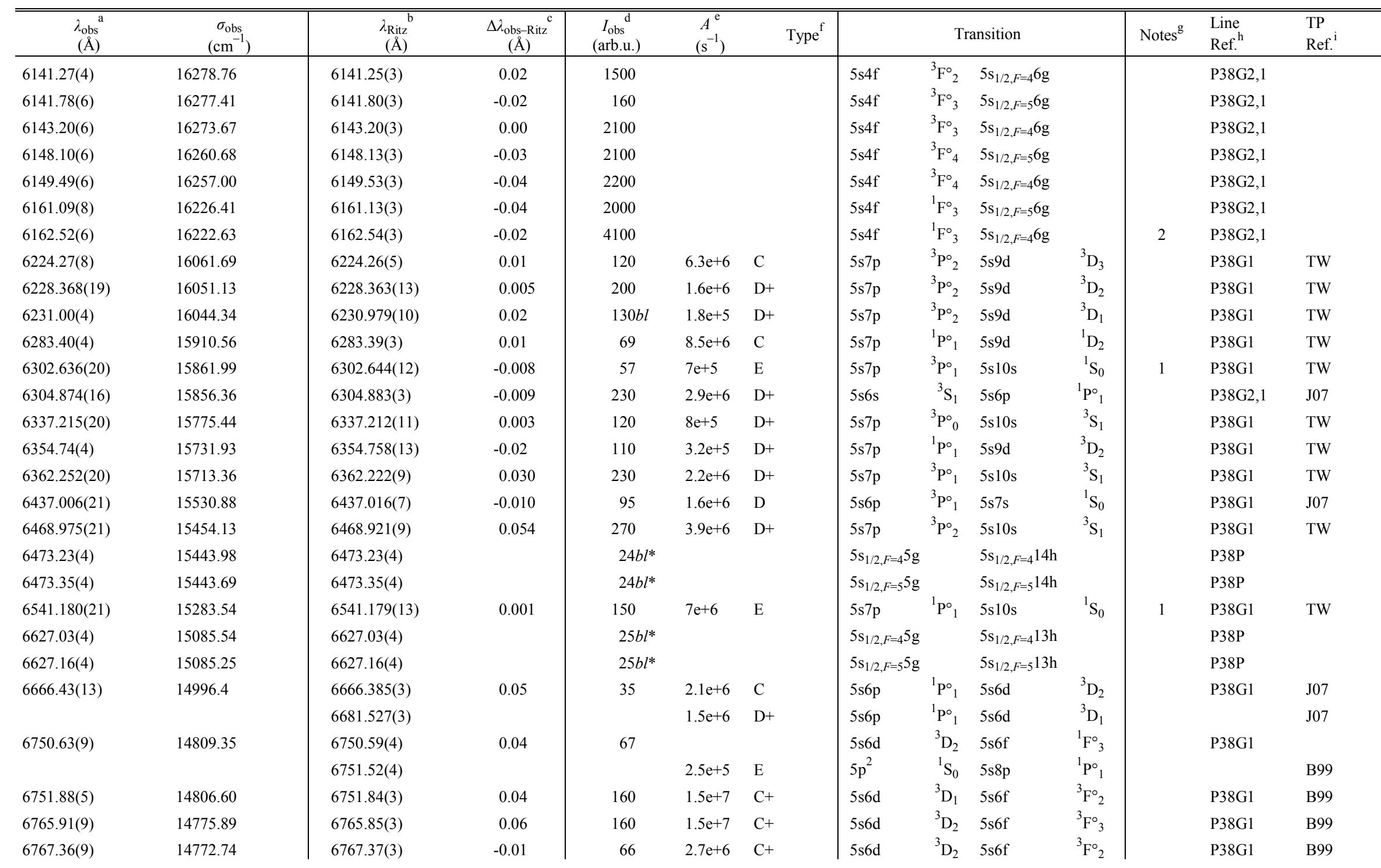


Volume 118 (2013) http://dx.doi.org/10.6028/jres.118.004

Journal of Research of the National Institute of Standards and Technology

\begin{tabular}{|c|c|c|c|c|c|c|c|c|c|c|c|c|}
\hline $\begin{array}{c}\lambda_{\text {obs }}{ }^{2} \\
(\AA)\end{array}$ & $\begin{array}{c}\sigma_{\mathrm{obs}} \\
\left(\mathrm{cm}^{-1}\right)\end{array}$ & $\begin{array}{c}\lambda_{\text {Ritz }} \mathrm{b} \\
(\AA)\end{array}$ & $\begin{array}{c}\Delta \lambda_{\text {obs-Ritz }}{ }^{c} \\
(\AA)\end{array}$ & $\begin{array}{c}I_{\text {obs }}^{\mathrm{d}} \\
\text { (arb.u.) }\end{array}$ & $\begin{array}{c}A^{\mathrm{e}} \\
\left(\mathrm{s}^{-1}\right)\end{array}$ & Type $^{\mathrm{f}}$ & \multicolumn{3}{|c|}{ Transition } & Notes $^{\mathrm{g}}$ & $\begin{array}{l}\text { Line } \\
\text { Ref. }^{h}\end{array}$ & $\begin{array}{l}\text { TP } \\
\text { Ref. }^{\mathrm{i}}\end{array}$ \\
\hline $6783.72(9)$ & 14737.11 & $6783.68(6)$ & 0.04 & 150 & $1.7 \mathrm{e}+7$ & $\mathrm{C}+$ & $5 \mathrm{~s} 6 \mathrm{~d}$ & $5 \mathrm{~s} 6 \mathrm{f}$ & ${ }^{3} \mathrm{~F}_{4}^{\circ}$ & & P38G1 & B99 \\
\hline $6790.42(5)$ & 14722.56 & $6790.42(3)$ & 0.00 & 45 & $1.9 \mathrm{e}+6$ & $\mathrm{C}+$ & $5 \mathrm{~s} 6 \mathrm{~d}$ & $5 s 6 f$ & ${ }^{3} \mathrm{~F}_{3}^{\circ}$ & & P38G1 & B99 \\
\hline $6831.65(7)$ & 14633.72 & $6831.65(7)$ & & $19 b l^{*}$ & & & $5 \mathrm{~s}_{1 / 2, F=4} 5 \mathrm{~g}$ & $5 \mathrm{~s}_{1 / 2, F=4} 12 \mathrm{~h}$ & & & P38G1 & \\
\hline $6831.78(7)$ & 14633.43 & $6831.78(7)$ & & $19 b l^{*}$ & & & $5 \mathrm{~s}_{1 / 2, F=5} 5 \mathrm{~g}$ & $5 \mathrm{~s}_{1 / 2, F=5} 12 \mathrm{~h}$ & & & P38G1 & \\
\hline $6841.03(7)$ & 14613.64 & $6841.04(4)$ & -0.01 & 6 & & & $5 \mathrm{~s} 5 \mathrm{f} \quad{ }^{3} \mathrm{~F}_{2}^{\circ}$ & $5 \mathrm{~s}_{1 / 2, F=4} 11 \mathrm{~g}$ & & & P38G1 & \\
\hline $6841.28(7)$ & 14613.11 & $6841.25(4)$ & 0.03 & 8 & & & $5 \mathrm{~s} 5 \mathrm{f}$ & $5 \mathrm{~s}_{1 / 2, F=5} 11 \mathrm{~g}$ & & & P38G1 & \\
\hline $6842.90(9)$ & 14609.65 & $6842.91(4)$ & -0.01 & 11 & & & $5 \mathrm{~s} 5 \mathrm{f}$ & $5 \mathrm{~s}_{1 / 2, F=4} 11 \mathrm{~g}$ & & & P38G1 & \\
\hline $6848.79(14)$ & 14597.1 & 6848.82(4) & -0.03 & 33 & & & $5 \mathrm{~s} 5 \mathrm{f}$ & $5 \mathrm{~s}_{1 / 2, F=5} 11 \mathrm{~g}$ & & & P38G1 & \\
\hline $6850.45(9)$ & 14593.56 & $6850.48(4)$ & -0.03 & 33 & & & $5 \mathrm{~s} 5 \mathrm{f}$ & $5 \mathrm{~s}_{1 / 2, F=4} 11 \mathrm{~g}$ & & & P38G1 & \\
\hline $6860.16(9)$ & 14572.90 & $6860.09(4)$ & 0.07 & 15 & & & $5 \mathrm{~s} 5 \mathrm{f}$ & $5 \mathrm{~s}_{1 / 2, F=5} 11 \mathrm{~g}$ & & & P38G1 & \\
\hline $6861.85(14)$ & 14569.3 & $6861.76(4)$ & 0.09 & 12 & & & $5 \mathrm{~s} 5 \mathrm{f}$ & $5 \mathrm{~s}_{1 / 2, F=4} 11 \mathrm{~g}$ & & & P38G1 & \\
\hline $6867.70(14)$ & 14556.9 & $6867.73(11)$ & -0.03 & 43 & $1.5 \mathrm{e}+6$ & $\mathrm{D}$ & $5 \mathrm{~s} 7 \mathrm{~s}$ & $5 \mathrm{~s} 8 \mathrm{p}$ & ${ }^{3} \mathrm{P}_{2}^{\circ}$ & & P38G1 & B99 \\
\hline $6891.5821(19)$ & 14506.454 & $6891.5826(15)$ & -0.0005 & 3900 & $6.5 \mathrm{e}+7$ & B & $5 \mathrm{~s} 6 \mathrm{~s}$ & $5 s 6 p$ & ${ }^{3} \mathrm{P}_{2}^{\circ}$ & & $\mathrm{K} 01 \mathrm{c}$ & $\mathrm{J} 07$ \\
\hline $6933.32(4)$ & 14419.13 & $6933.343(23)$ & -0.02 & 34 & $1.3 \mathrm{e}+6$ & $\mathrm{D}$ & $5 \mathrm{~s} 7 \mathrm{~s}$ & $5 \mathrm{~s} 8 \mathrm{p}$ & ${ }^{3} \mathrm{P}^{\circ}{ }_{1}$ & & P38G1 & B99 \\
\hline & & $6952(5)$ & & & $1.4 \mathrm{e}+6$ & $\mathrm{D}$ & $5 \mathrm{~s} 7 \mathrm{~s}$ & $5 \mathrm{~s} 8 \mathrm{p}$ & ${ }^{3} \mathrm{P}_{0}^{\circ}$ & & & B99 \\
\hline 7108.57(8) & 14063.64 & $7108.572(21)$ & 0.00 & 29 & & & $5 \mathrm{~s} 6 \mathrm{~d} \quad{ }^{1} \mathrm{D}_{2}$ & $5 \mathrm{~s} 9 \mathrm{p}$ & ${ }^{3} \mathrm{P}_{1}^{\circ}$ & & P38G1 & \\
\hline 7113.99(10) & 14052.93 & $7113.99(10)$ & & $66 b l^{*}$ & & & $5 \mathrm{~s}_{1 / 2, F=4} 5 \mathrm{~g}$ & $5 \mathrm{~s}_{1 / 2, F=4} 11 \mathrm{~h}$ & & & P38G1 & \\
\hline 7114.14(10) & 14052.64 & 7114.14(10) & & $66 b l^{*}$ & & & $5 \mathrm{~s}_{1 / 2, F=5} 5 \mathrm{~g}$ & $5 \mathrm{~s}_{1 / 2, F=5} 11 \mathrm{~h}$ & & & P38G1 & \\
\hline $7182.9038(21)$ & 13918.110 & $7182.9048(15)$ & -0.0010 & 5800 & $5.6 \mathrm{e}+7$ & $\mathrm{~B}$ & $5 \mathrm{~s} 6 \mathrm{~s}$ & $5 \mathrm{~s} 6 \mathrm{p}$ & ${ }^{3} \mathrm{P}_{1}^{\circ}$ & 2 & $\mathrm{~K} 01 \mathrm{c}$ & J07 \\
\hline $7218.22(10)$ & 13850.02 & $7218.15(4)$ & 0.07 & 14 & & & $5 \mathrm{~s} 5 \mathrm{f}$ & $5 \mathrm{~s}_{1 / 2, F=5} 10 \mathrm{~g}$ & & & P38G1 & \\
\hline $7219.96(10)$ & 13846.67 & $7219.99(3)$ & -0.03 & 14 & & & $5 \mathrm{~s} 5 \mathrm{f}$ & $5 \mathrm{~s}_{1 / 2, F=4} 10 \mathrm{~g}$ & & & P38G1 & \\
\hline $7220.20(10)$ & 13846.22 & $7220.23(4)$ & -0.03 & 25 & & & $5 \mathrm{~s} 5 \mathrm{f}$ & $5 \mathrm{~s}_{1 / 2, F=5} 10 \mathrm{~g}$ & & & P38G1 & \\
\hline $7222.05(10)$ & 13842.67 & $7222.07(4)$ & -0.02 & 71 & & & $5 \mathrm{~s} 5 \mathrm{f}$ & $5 \mathrm{~s}_{1 / 2, F=4} 10 \mathrm{~g}$ & & & P38G1 & \\
\hline $7228.63(10)$ & 13830.07 & $7228.66(4)$ & -0.03 & 74 & & & $5 \mathrm{~s} 5 \mathrm{f}$ & $5 \mathrm{~s}_{1 / 2, F=5} 10 \mathrm{~g}$ & & & P38G1 & \\
\hline $7230.44(10)$ & 13826.61 & $7230.51(3)$ & -0.07 & 61 & & & $5 \mathrm{~s} 5 \mathrm{f}$ & $5 \mathrm{~s}_{1 / 2, F=4} 10 \mathrm{~g}$ & & & P38G1 & \\
\hline $7241.23(10)$ & 13806.00 & $7241.22(4)$ & 0.01 & 50 & & & $5 \mathrm{~s} 5 \mathrm{f}$ & $5 \mathrm{~s}_{1 / 2, F=5} 10 \mathrm{~g}$ & & & P38G1 & \\
\hline $7243.06(10)$ & 13802.51 & $7243.07(4)$ & -0.01 & 34 & & & $5 \mathrm{~s} 5 \mathrm{f}$ & $5 \mathrm{~s}_{1 / 2, F=4} 10 \mathrm{~g}$ & & & P38G1 & \\
\hline 7254.981(11) & 13779.836 & $7254.982(6)$ & -0.001 & 170 & $1.17 \mathrm{e}+7$ & B & $5 \mathrm{~s} 6 \mathrm{p}$ & $5 \mathrm{~s} 7 \mathrm{~s}$ & ${ }^{3} \mathrm{~S}_{1}$ & & $\mathrm{~K} 01 \mathrm{c}$ & J07 \\
\hline 7272.04(11) & 13747.51 & $7272.05(7)$ & -0.01 & 15 & & & $5 \mathrm{~s} 7 \mathrm{~d}$ & $5 \mathrm{~s} 11 \mathrm{f}$ & ${ }^{1} \mathrm{~F}_{3}^{\circ}$ & & P38G1 & \\
\hline $7273.07(11)$ & 13745.56 & $7273.07(7)$ & 0.00 & 15 & & & $5 \mathrm{~s} 7 \mathrm{~d}$ & $5 \mathrm{~s} 11 \mathrm{f}$ & ${ }^{3} \mathrm{~F}_{2}^{\circ}$ & & P38G1 & \\
\hline
\end{tabular}


Volume 118 (2013) http://dx.doi.org/10.6028/jres.118.004

Journal of Research of the National Institute of Standards and Technology

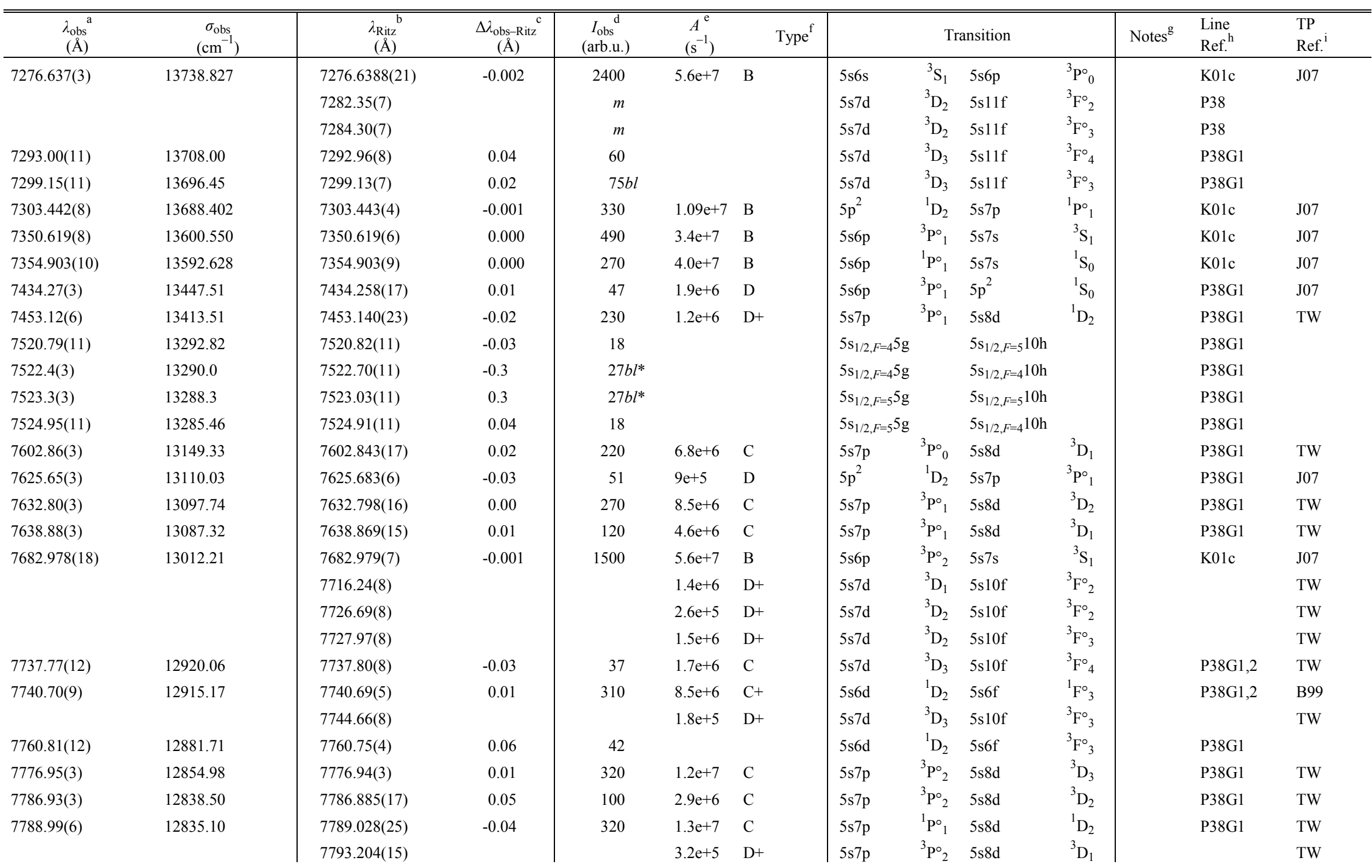


Volume 118 (2013) http://dx.doi.org/10.6028/jres.118.004

Journal of Research of the National Institute of Standards and Technology

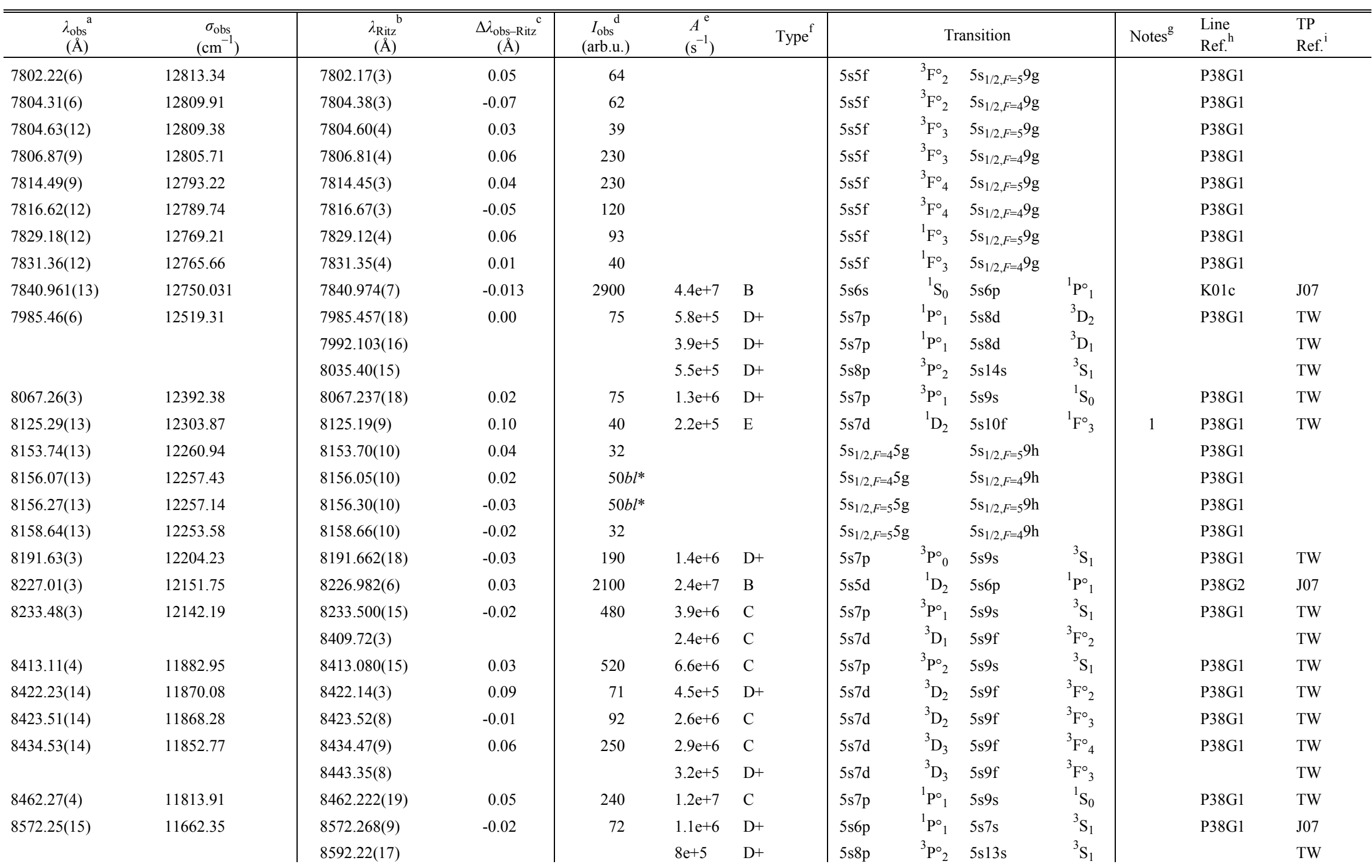


Volume 118 (2013) http://dx.doi.org/10.6028/jres.118.004

Journal of Research of the National Institute of Standards and Technology

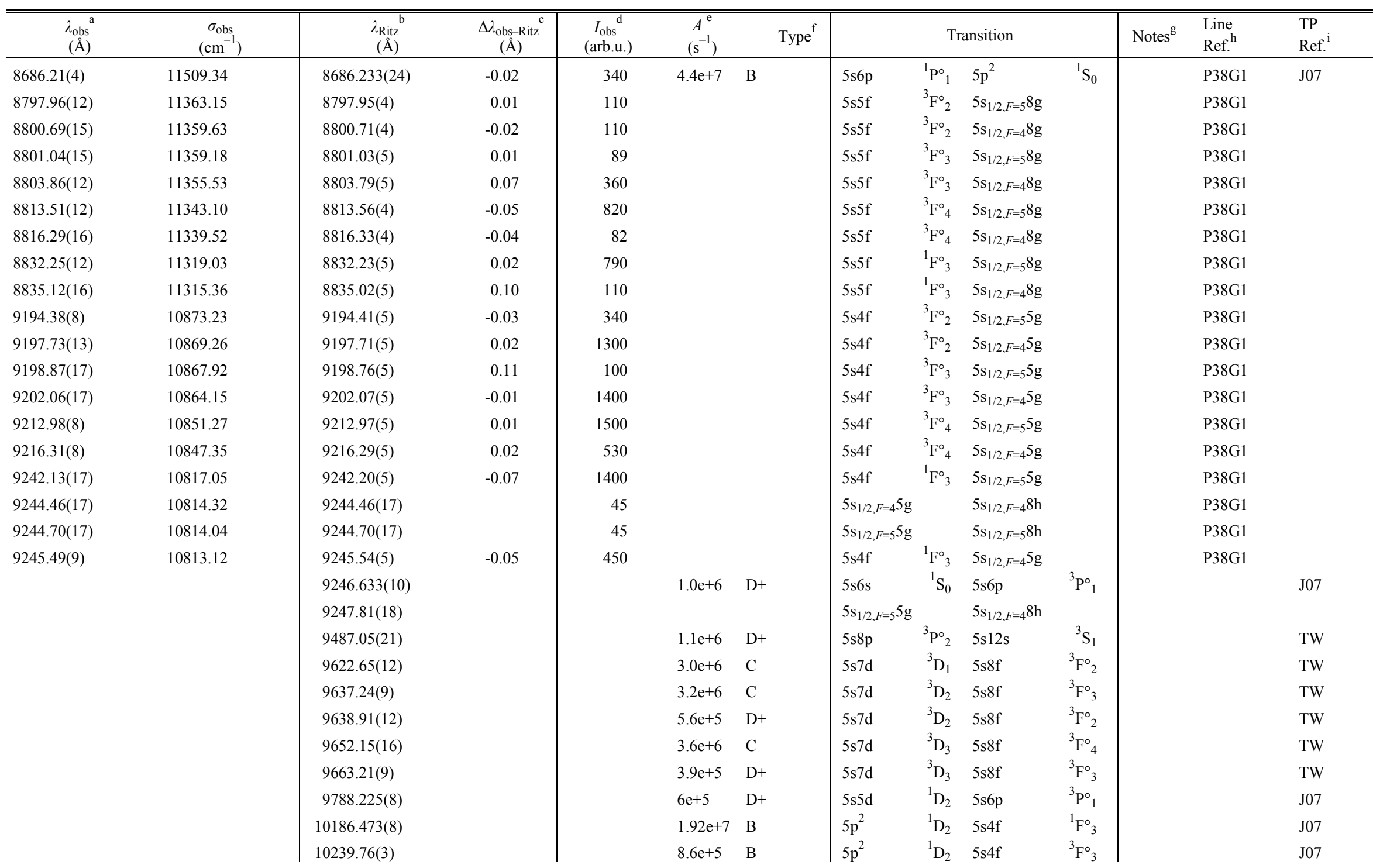


Volume 118 (2013) http://dx.doi.org/10.6028/jres.118.004

Journal of Research of the National Institute of Standards and Technology

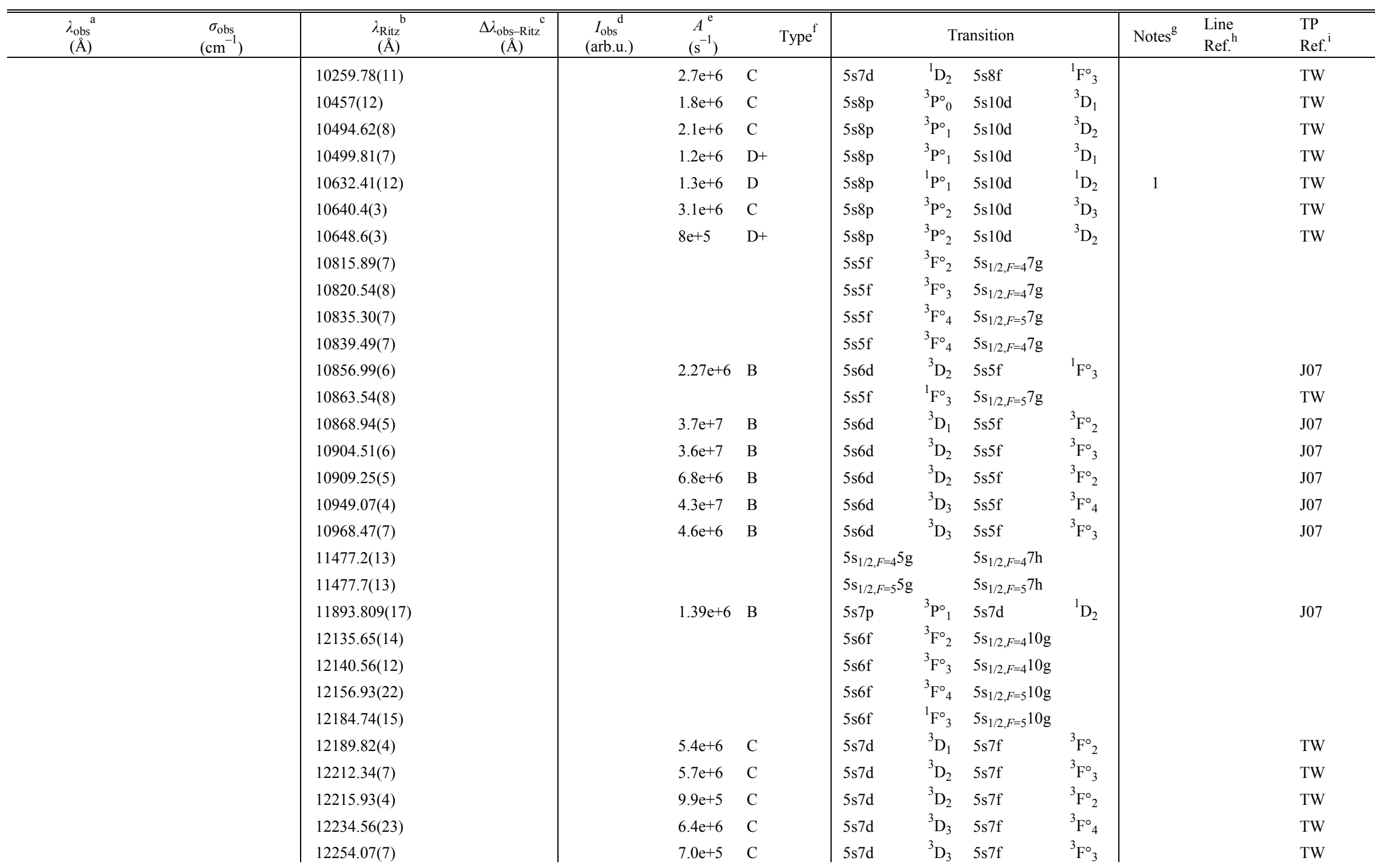


Volume 118 (2013) http://dx.doi.org/10.6028/jres.118.004

Journal of Research of the National Institute of Standards and Technology

\begin{tabular}{|c|c|c|c|c|c|c|c|c|c|c|c|}
\hline $\begin{array}{c}\lambda_{\text {obs }}{ }^{a} \\
(\AA)\end{array}$ & $\begin{array}{c}\sigma_{\mathrm{obs}} \\
\left(\mathrm{cm}^{-1}\right)\end{array}$ & $\begin{array}{c}\lambda_{\text {Ritz }}{ }^{\prime} \\
(\AA)\end{array}$ & $\begin{array}{c}\Delta \lambda_{\text {obs-Ritz }}{ }^{c} \\
(\AA)\end{array}$ & $\begin{array}{c}I_{\text {obs }}{ }^{d} \\
\text { (arb.u.) }\end{array}$ & $\begin{array}{l}A^{\mathrm{e}} \\
\left(\mathrm{s}^{-1}\right)\end{array}$ & Type $^{\text {f }}$ & \multicolumn{2}{|c|}{ Transition } & Notes $^{\mathrm{g}}$ & \multirow[t]{2}{*}{$\begin{array}{l}\text { Line } \\
\text { Ref. }\end{array}$} & $\begin{array}{l}\text { TP } \\
\text { Ref. }\end{array}$ \\
\hline & & $12687.0(3)$ & & & & & $5 \mathrm{~s}_{1 / 2, F=4} 6 \mathrm{~g}$ & $5 \mathrm{~s}_{1 / 2, F=4} 10 \mathrm{~h}$ & & & \\
\hline & & $12687.7(3)$ & & & & & $5 \mathrm{~s}_{1 / 2, F=5} 6 \mathrm{~g}$ & $5 \mathrm{~s}_{1 / 2, F=5} 10 \mathrm{~h}$ & & & \\
\hline & & $12693.0(3)$ & & & & & $5 \mathrm{~s}_{1 / 2, F=5} 6 \mathrm{~g}$ & $5 \mathrm{~s}_{1 / 2, F=4} 10 \mathrm{~h}$ & & & \\
\hline & & $12772.783(18)$ & & & $2.38 \mathrm{e}+7$ & B & $5 \mathrm{~s} 7 \mathrm{p} \quad{ }^{1} \mathrm{P}^{\circ}{ }_{1}$ & $5 \mathrm{~s} 7 \mathrm{~d}$ & & & J07 \\
\hline & & $12827.51(4)$ & & & $1.6 \mathrm{e}+7$ & $\mathrm{C}+$ & $5 \mathrm{~s} 7 \mathrm{p}$ & $5 \mathrm{~s} 7 \mathrm{~d}$ & & & $\mathrm{~J} 07$ \\
\hline & & $12901.147(21)$ & & & $2.11 \mathrm{e}+7$ & B & $5 \mathrm{~s} 7 \mathrm{p}$ & $5 \mathrm{~s} 7 \mathrm{~d}$ & & & J07 \\
\hline & & $12930.400(22)$ & & & $1.15 \mathrm{e}+7$ & $\mathrm{C}+$ & $5 \mathrm{~s} 7 \mathrm{p}$ & $5 \mathrm{~s} 7 \mathrm{~d}$ & & & J07 \\
\hline & & $13115.86(14)$ & & & $7.8 \mathrm{e}+5$ & $\mathrm{C}$ & $5 \mathrm{~s} 8 \mathrm{p}$ & $5 \mathrm{~s} 9 \mathrm{~d}$ & & & TW \\
\hline & & 13144.73(4) & & & $2.9 \mathrm{e}+5$ & $\mathrm{D}$ & $5 \mathrm{~s} 5 \mathrm{~d}$ & $5 \mathrm{~s} 6 \mathrm{p}$ & & & J07 \\
\hline & & $13153.64(25)$ & & & $9.8 \mathrm{e}+5$ & $\mathrm{C}$ & $5 \mathrm{~s} 8 \mathrm{~d}$ & $5 \mathrm{~s} 10 \mathrm{f}$ & & & TW \\
\hline & & $13175.40(24)$ & & & $1.0 \mathrm{e}+6$ & $\mathrm{C}$ & $5 \mathrm{~s} 8 \mathrm{~d}$ & $5 \mathrm{~s} 10 \mathrm{f}$ & & & TW \\
\hline & & $13184.0(3)$ & & & $1.2 \mathrm{e}+6$ & $\mathrm{C}$ & $5 \mathrm{~s} 8 \mathrm{~d}$ & $5 \mathrm{~s} 10 \mathrm{f}$ & & & TW \\
\hline & & $13224.87(13)$ & & & $4.9 \mathrm{e}+6$ & $\mathrm{C}$ & $5 \mathrm{~s} 7 \mathrm{~d}$ & $5 \mathrm{~s} 7 \mathrm{f}$ & & & TW \\
\hline & & $13298.074(16)$ & & & $2.9 \mathrm{e}+7$ & B & $5 \mathrm{~s} 7 \mathrm{p}$ & $5 \mathrm{~s} 7 \mathrm{~d}$ & & & J07 \\
\hline & & $13347.570(16)$ & & & $7.3 \mathrm{e}+6$ & $\mathrm{C}+$ & $5 \mathrm{~s} 7 \mathrm{p}$ & $5 \mathrm{~s} 7 \mathrm{~d}$ & & & J07 \\
\hline & & 13373(20) & & & $2.5 \mathrm{e}+6$ & $\mathrm{C}$ & $5 \mathrm{~s} 8 \mathrm{p}$ & $5 \mathrm{~s} 9 \mathrm{~d}$ & & & TW \\
\hline & & $13378.885(18)$ & & & $8.1 \mathrm{e}+5$ & $\mathrm{C}$ & $5 \mathrm{~s} 7 \mathrm{p}$ & $5 \mathrm{~s} 7 \mathrm{~d}$ & & & J07 \\
\hline & & $13430.71(10)$ & & & $3.0 \mathrm{e}+6$ & $\mathrm{C}$ & $5 \mathrm{~s} 8 \mathrm{p}$ & $5 \mathrm{~s} 9 \mathrm{~d}$ & & & TW \\
\hline & & $13442.88(10)$ & & & $1.6 \mathrm{e}+6$ & $\mathrm{C}$ & $5 \mathrm{~s} 8 \mathrm{p}$ & $5 \mathrm{~s} 9 \mathrm{~d}$ & & & TW \\
\hline & & $13533.67(20)$ & & & $4.8 \mathrm{e}+6$ & $\mathrm{C}$ & $5 \mathrm{~s} 8 \mathrm{p}$ & $5 \mathrm{~s} 9 \mathrm{~d}$ & & & TW \\
\hline & & $13664.2(5)$ & & & $4.3 \mathrm{e}+6$ & $\mathrm{C}$ & $5 \mathrm{~s} 8 \mathrm{p}$ & $5 \mathrm{~s} 9 \mathrm{~d}$ & & & TW \\
\hline & & $13668.85(9)$ & & & $3.0 \mathrm{e}+7$ & B & $5 \mathrm{~s} 6 \mathrm{~d}$ & $5 \mathrm{~s} 5 \mathrm{f}$ & & & J07 \\
\hline & & $13683.9(4)$ & & & $1.1 \mathrm{e}+6$ & $\mathrm{C}$ & $5 \mathrm{~s} 8 \mathrm{p}$ & $5 \mathrm{~s} 9 \mathrm{~d}$ & & & TW \\
\hline & & $13744.26(10)$ & & & $1.81 \mathrm{e}+6$ & $\mathrm{~B}$ & $5 \mathrm{~s} 6 \mathrm{~d}$ & $5 \mathrm{~s} 5 \mathrm{f}$ & & & J07 \\
\hline & & $13882.94(17)$ & & & & & $5 s 6 f$ & $5 \mathrm{~s}_{1 / 2, F=4} 9 \mathrm{~g}$ & & & \\
\hline & & $13889.37(15)$ & & & & & $5 \mathrm{~s} 6 \mathrm{f}$ & $5 \mathrm{~s}_{1 / 2, F=4} 9 \mathrm{~g}$ & & & \\
\hline & & 13910.6(3) & & & & & $5 s 6 f$ & $5 \mathrm{~s}_{1 / 2, F=5} 9 \mathrm{~g}$ & & & \\
\hline & & $13941.828(22)$ & & & $1.15 \mathrm{e}+6$ & $\mathrm{C}+$ & $5 \mathrm{~s} 7 \mathrm{p}$ & $5 \mathrm{~s} 7 \mathrm{~d}$ & & & J07 \\
\hline & & $13947.05(17)$ & & & & & $5 s 6 f$ & $5 \mathrm{~s}_{1 / 2, F=5} 9 \mathrm{~g}$ & & & \\
\hline & & $13975.997(23)$ & & & $8.3 \mathrm{e}+5$ & $\mathrm{C}+$ & $5 \mathrm{~s} 7 \mathrm{p}$ & $5 \mathrm{~s} 7 \mathrm{~d}$ & & & $\mathrm{~J} 07$ \\
\hline
\end{tabular}


Volume 118 (2013) http://dx.doi.org/10.6028/jres.118.004

Journal of Research of the National Institute of Standards and Technology

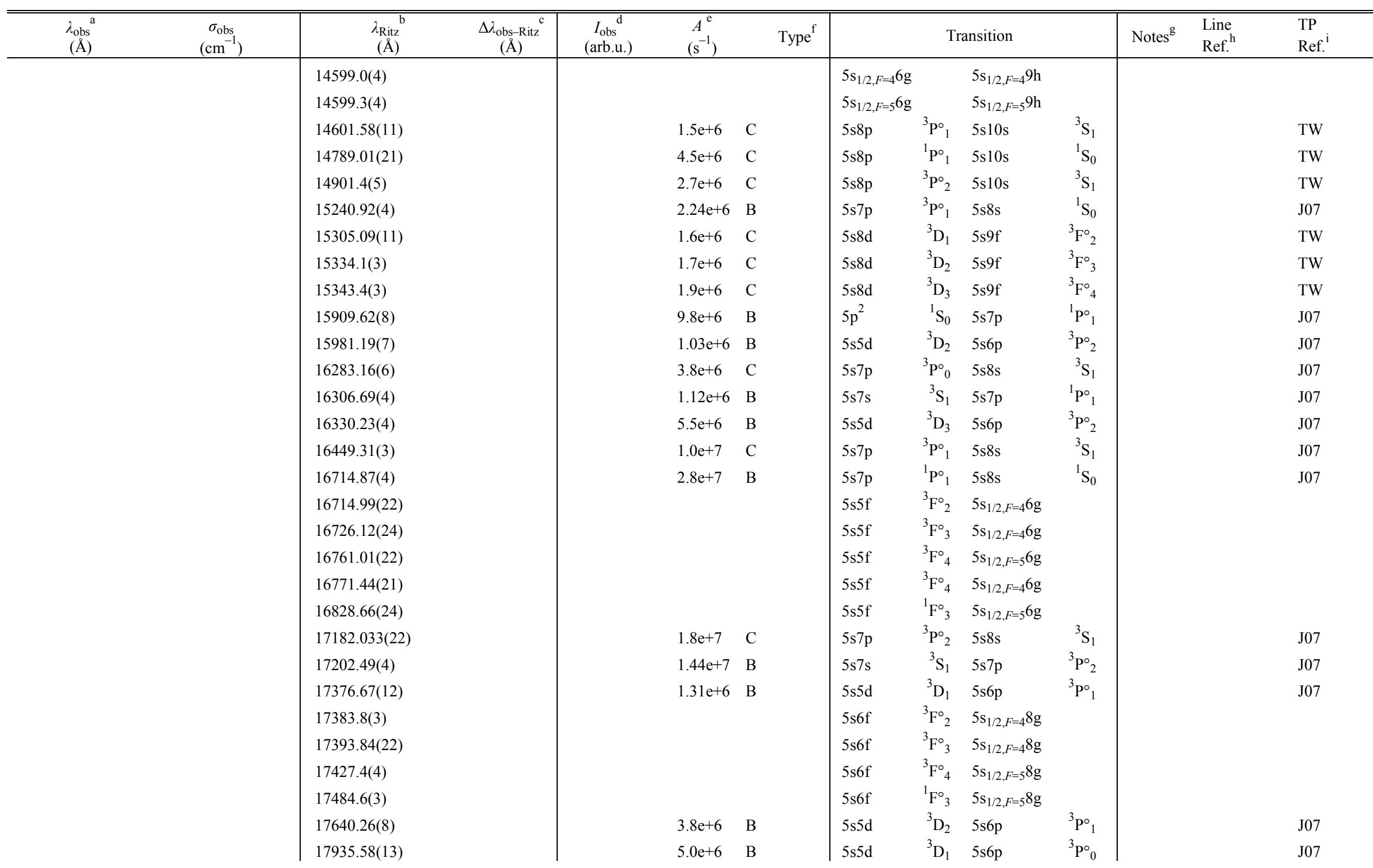


Volume 118 (2013) http://dx.doi.org/10.6028/jres.118.004

Journal of Research of the National Institute of Standards and Technology

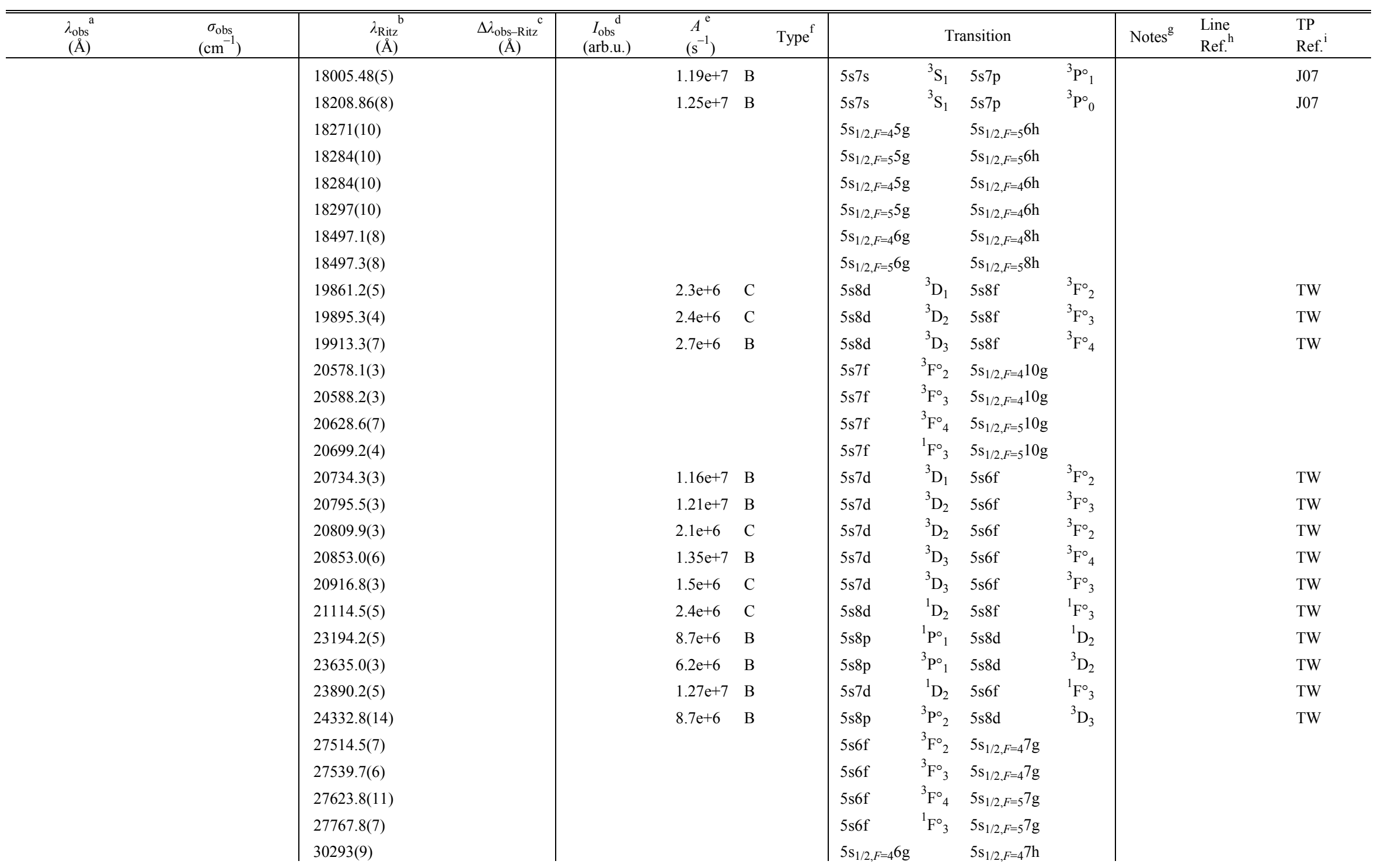


Volume 118 (2013) http://dx.doi.org/10.6028/jres.118.004

Journal of Research of the National Institute of Standards and Technology

\begin{tabular}{|c|c|c|c|c|c|c|c|c|c|c|}
\hline $\begin{array}{l}\lambda_{\text {obs }}{ }^{a} \\
(\AA) \\
\end{array}$ & $\begin{array}{c}\sigma_{\mathrm{obs}} \\
\left(\mathrm{cm}^{-1}\right)\end{array}$ & $\begin{array}{c}\lambda_{\text {Ritz }}{ }^{b} \\
(\AA) \\
\end{array}$ & $\begin{array}{c}\Delta \lambda_{\text {obs-Ritz }}{ }^{c} \\
(\AA)\end{array}$ & $\begin{array}{c}I_{\mathrm{obs}}^{\mathrm{d}} \\
\text { (arb.u.) } \\
\end{array}$ & $\begin{array}{c}A^{\mathrm{e}} \\
\left(\mathrm{s}^{-1}\right) \\
\end{array}$ & Type $^{f}$ & Transition & Notes $^{\mathrm{g}}$ & $\begin{array}{l}\text { Line } \\
\text { Ref. }^{h}\end{array}$ & $\begin{array}{l}\text { TP } \\
\text { Ref. }^{\text {i }}\end{array}$ \\
\hline & & $30295(9)$ & & & & & $5 \mathrm{~s}_{1 / 2, F=5} 6 \mathrm{~g}$ & & & \\
\hline
\end{tabular}

${ }^{\text {a }}$ Observed wavelengths below $2000 \AA$ are given in vacuum, above that in standard air. Conversion from vacuum to air wavelengths was made using the five-parameter formula from Peck and Reeder [28].

Estimated one-standard-deviation uncertainties are given in parentheses after the value in units of the least significant figure. If two uncertainties are given, the first is a statistical uncertainty, and the second

is systematic. Uncertainties given for the air wavelengths do not include uncertainties of the air-vacuum conversion formula.

${ }^{b}$ Ritz wavelengths and their uncertainties were determined in the level optimization procedure using the LOPT code [23].

${ }^{\mathrm{c}}$ Deviation of the observed wavelength from the Ritz value. This column is blank for lines that alone determine one of the two energy levels involved in the transition.

${ }^{\mathrm{d}}$ Observed relative intensities have been converted to a uniform scale corresponding to emission from a plasma with an effective excitation temperature of about 3 eV (see Sec. 6.6).

${ }^{\mathrm{e}}$ Transition probability values are followed by a letter code denoting the estimated uncertainty (see Table 3 ).

${ }_{\mathrm{f}}^{\mathrm{f}}$ Designations of transition types are as follows: E2 - electric quadrupole transition (enabled by an external electric field; see Sec. 2.3); HF - transition induced by hyperfine interaction.

${ }^{\mathrm{g}}$ Notes: 1 - Transition probability value determined in the present work is uncertain because of strong cancellation effects see Sec. 7); 2 - Observed intensity value is unreliable (see Sec. 6.6); 3 - Observed intensity was marked as questionable in Paschen and Campbell [2].

${ }^{\mathrm{h}}$ Codes for references to observed wavelengths and line identifications: B69 - Bhatia [5]; K01c - Karlsson and Litzén [11] (observed wavelengths have been corrected to account for a minor systematic error in the calibration of the wave number scale; see Sec. 2.1); L31 - Lang and Sawyer [3]; L93c - Larkins and Hannaford [8] (re-calibrated using the measurement of Wang et al. [27]; see Sec. 2.2); P38 - Paschen and Campbell [2]; this code followed by "P", "TP", "G1", "G2", etc. means the measurements made with a one-prism spectrograph, three-prism spectrograph, and a grating spectrograph in various orders of diffraction (see Sec. 2.3); W96 - lines identified by Wagatsuma [15] (no wavelength measurement was given, but the intensities were measured; see Sec. 2.6); Z01 - von Zanthier et al. [10].

Codes for references to transition probability values: A86 - Ansbacher et al. [38]; A86c - The $A$-value was derived from the radiative lifetime measured by Ansbacher $e$ t al. [38] using transition rates for

weaker decay branches calculated by Jönsson and Andersson [43] (see Sec. 7); B99 - Biémont and Zeippen [35]; B01 - Becker et al. [37]; CMEF00 - Curtis et al. [39]; J07 - Jönsson and Andersson [43]; L94 - Lavín and Martin [44]; M96 - Martinez et al. [41]; TW - This work. 
breadth of the hfs. Thus, even if the centers of gravity of the observed hfs are determined precisely, we can expect them to deviate from the Ritz values due to the intrinsic inaccuracy of the distinctive energy levels.

We can also expect the lines from the category $b$ of Karlsson and Litzén to have the largest uncertainties due to inaccuracies of the measurement of centers of gravity of the hfs.

To estimate the wave number uncertainties, as a zero approximation, I neglected the hfs considerations and assumed that the measurement uncertainties $\delta \sigma$ are entirely due to statistical and systematic uncertainties ( $\delta \sigma_{\text {stat }}$ and $\delta \sigma_{\text {syst }}$, respectively) in the wave number measurements of symmetric well-resolved features (see, for example, Kramida and Nave [22]:

$$
\begin{aligned}
& \delta \sigma \approx\left(\delta{\sigma_{\text {stat }}}^{2}+\delta \sigma_{\text {syst }}^{2}\right)^{1 / 2}, \\
& \delta \sigma_{\text {stat }} \approx W /(2 S / N),
\end{aligned}
$$

where $W$ is the full width at half-maximum. The $S / N$ values are given in Table 1 of Karlsson and Litzén [11] in the column of line intensities. The values of $W$ and $\delta \sigma_{\text {syst }}$ can be estimated as $0.02 \mathrm{~cm}^{-1}$ and $0.003 \mathrm{~cm}^{-1}$, respectively, from the statement that the uncertainty of the wave numbers varies from $\pm 0.003 \mathrm{~cm}^{-1}$ for strong unblended lines to $\pm 0.02 \mathrm{~cm}^{-1}$ for weak, unresolved, or blended lines [11]. However, I found that setting

$$
W=0.03 \mathrm{~cm}^{-1}
$$

results in better statistical consistency of the energy levels with the measured wave numbers. The value of $0.003 \mathrm{~cm}^{-1}$ for the systematic uncertainty corresponds to the maximum value of the wavenumber scale correction described above. With these assumed wavenumber uncertainty values, using the leveloptimization code LOPT [23], I have derived a set of optimized energy levels from the observed wave numbers given in Table 1 of Karlsson and Litzén [11]. The resulting values agreed with those given in Ref. [11] with an average deviation of $0.003 \mathrm{~cm}^{-1}$, the maximum deviation being $0.006 \mathrm{~cm}^{-1}$. This close agreement indicates that the assumed wavenumber uncertainties are close to those used by Karlsson and Litzén in their level-optimization procedure.

The final assignment of wavenumber uncertainties was made when all measured lines from different sets of measurements were brought together in a comprehensive line list and checked for internal consistency (see Sec. 3). At that stage, it was found that the lines from the categories a and b of Karlsson and Litzén [11] deviate from the Ritz values by much greater amounts than those of the category c, which is consistent with the expectations noted above. Therefore, the uncertainties of these categories of lines have been increased by a factor of 3.7 as compared to Eq. (3). Only then can the measurements of Karlsson and Litzén [11] be considered consistent with those of Paschen and Campbell [2] and with other observations.

\subsection{Measurements of Larkins and Hannaford [8]}

Larkins and Hannaford [8] measured the absolute frequencies of hyperfine components of the four lines at $1936 \AA\left(5 \mathrm{~s} 5 \mathrm{p}^{3} \mathrm{P}_{0}^{\circ}{ }_{0}-5 \mathrm{~s} 6 \mathrm{~s}{ }^{3} \mathrm{~S}_{1}\right), 1977 \AA\left(5 \mathrm{~s} 5 \mathrm{p}^{3} \mathrm{P}^{\circ}{ }_{1}-5 \mathrm{~s} 6 \mathrm{~s}{ }^{3} \mathrm{~S}_{1}\right), 2079 \AA\left(5 \mathrm{~s} 5 \mathrm{p}^{3} \mathrm{P}^{\circ}{ }_{2}-5 \mathrm{~s} 6 \mathrm{~s}{ }^{3} \mathrm{~S}_{1}\right)$, and $2306 \AA$ $\left(5 \mathrm{~s}^{2}{ }^{1} \mathrm{~S}_{0}-5 \mathrm{~s} 5 \mathrm{p}^{3} \mathrm{P}^{\circ}{ }_{1}\right)$ emitted by a hollow-cathode lamp by using a $2.5 \mathrm{~m}$ scanning monochromator. The monochromator was fitted with a 316 lines $/ \mathrm{mm}$ échelle grating having a blaze angle of $65^{\circ}$ and mounted in a Czerny-Turner configuration. The four In II lines listed above were measured in different orders of diffraction $\left(29^{\text {th }}\right.$ for the first two lines, $27^{\text {th }}$ for the third one, and $24^{\text {th }}$ for the fourth). The absolute frequency scale was calibrated separately for each of the four lines using different sets of Fe I and Fe II reference lines, for which the Ritz wavelengths from Nave et al. [19] and O'Brian et al. [24] were used. The orders of diffraction for the reference lines were different for different Fe lines and, most importantly, for different In II lines. Larkins and Hannaford assumed the calibration uncertainty to be equal to the quadrature sum of the quoted uncertainties in the energies of the upper and lower Fe levels of the reference lines [19,24]. However, as noted by Reader [25], this method of calibration that uses reference lines in an order of diffraction different from that of the measured line can cause a significant systematic error in a CzernyTurner spectrometer. These systematic errors are the most probable cause of the discrepancies between the 
wave numbers measured by Larkins and Hannaford [8] and by Karlsson and Litzén [11] mentioned in the previous section. The suspected culprit of these systematic errors is the variation of the refraction index of air with pressure. Larkins and Hannaford made all measurements in air and used a formula for conversion from air wavelengths to vacuum wave numbers that does not account for the air pressure, temperature, and composition. They did not mention anything about the air conditions in their setting. If the grating is assumed to be ideal, the observed wavelength of any line in any order of diffraction is equal to the wavelength in the first order multiplied by the order number. However, the refraction index of air is different at different first-order wavelengths. Thus, if the reference and investigated lines are observed in different orders of diffraction, the variation of the refraction index of air has to be taken into account in the derivation of the measured wavelength. My numerical experiments showed that possible variations of the atmospheric air pressure between $101.2 \mathrm{kPa}$ and $104.8 \mathrm{kPa}$ in the experiment of Larkins and Hannaford [8] can explain all discrepancies between their measurements and those of Karlsson and Litzén [11] (the average air pressure at the site of measurements in Clayton, Australia, is $101.11 \mathrm{kPa}$ ).

It should be noted that the Fe I and Fe II wave numbers given in Refs. $[19,24]$ were calibrated against the Ar II reference lines from Norlén [17] and thus have to be increased by 6.7 parts in $10^{8}$ (see the discussion in the previous section). The difference between the reference Fe wavenumbers as given by Larkins and Hannaford from the corrected ones given in the current version of the NIST Atomic Spectra Database [26] amounts to $0.004 \mathrm{~cm}^{-1}$, which is much smaller than the discrepancies in the measured In II wave numbers.

In the absence of reliable means to correct the calibration errors of Larkins and Hannaford [8], the measurements of Karlsson and Litzén [11] should be preferred, despite the fact that they [11] possess significantly greater statistical uncertainties. However, for one transition, $5 \mathrm{~s}^{2} \mathrm{~S}_{0}-5 \mathrm{~s} 5 \mathrm{p}{ }^{3} \mathrm{P}_{1}$, the measurement of Larkins and Hannaford [8] can be re-calibrated using a recent independent high-precision measurement of an hfs component of this line. Namely, Wang et al. [27] measured the absolute frequency of the $5 \mathrm{~s}^{2}{ }^{1} \mathrm{~S}_{0}(\mathrm{~F}=9 / 2)-5 \mathrm{~s} 5 \mathrm{p}^{3} \mathrm{P}_{1}(\mathrm{~F}=11 / 2)$ transition in a single ${ }^{115} \mathrm{In}^{+}$ion cooled in a radio-frequency trap by using a frequency comb referenced to a Cs atomic clock. Converted to wave number units, their result is $43351.622760(3) \mathrm{cm}^{-1}$. The wave number of this transition, as measured by Larkins and Hannaford [8], is $43351.614(2) \mathrm{cm}^{-1}$. The difference is $0.009 \mathrm{~cm}^{-1}$, which is almost twice as large as the systematic uncertainty assumed by Larkins and Hannaford. By utilizing the air wavelengths of the three hfs components of the $5 \mathrm{~s}^{2}{ }^{1} \mathrm{~S}_{0}-5 \mathrm{~s} 5 \mathrm{p}{ }^{3} \mathrm{P}_{1}$ transition, which are given by Larkins and Hannaford with relatively greater precision, I obtained $43350.5817(15) \mathrm{cm}^{-1}$ for the corrected wave number of the centroid of this transition.

\subsection{Measurements of Paschen and Campbell [2]}

Paschen and Campbell [2] observed the In II spectrum excited in the negative glow of a hollow carbon cathode discharge filled with helium. The spectrum was dispersed in the region $2350 \AA$ through $9217 \AA$ using a $4 \mathrm{~m}$ concave grating spectrograph in various orders of diffraction (first through eighth) and recorded on photographic plates. Additional measurements of weaker lines were made with a higherthroughput single-prism spectrograph in the region $2078 \AA$ through $6627 \AA$ and a three-prism spectrograph in the region $3802 \AA$ through $4229 \AA$. The wavelength scale was calibrated against known lines of helium, neon, and iron. Paschen and Campbell listed about 1400 measured wave numbers of lines belonging to about 500 transitions of In II. Many of the lines had completely resolved hfs patterns, but most of them were resolved only partially. For all lines, Paschen and Campbell gave an estimated center-of-gravity wavenumber (averaged over the hyperfine structure) without an uncertainty estimate. Thus, in order to obtain an optimized set of energy levels, it is necessary to estimate the uncertainty for each line.

The number of decimal places in the wave numbers listed by Paschen and Campbell can serve as a guide to their uncertainties. Most of the wave numbers of hfs components in the red region of the spectrum measured with the grating spectrograph were given with three digits after the decimal point. However, the center-of-gravity wave numbers were given with only two digits after the point, which indicates a reasonable loss of accuracy in the averaging. The nominal resolution of the grating spectrograph was $R \equiv \lambda / \Delta \lambda \approx 82500$ in the first order of diffraction [2], so one can expect the uncertainty of the wave numbers $\sigma$ of individual hfs components to be greater than approximately $0.1 \sigma / R \approx 0.013 \mathrm{~cm}^{-1}$ at the longest 
wavelength $9217 \AA$. The finite entrance slit width and uncertainties of reference lines should further increase the uncertainty. For far-ultraviolet lines measured with the prism spectrograph, the wave numbers are given with only one digit after the decimal point, indicating a much greater uncertainty.

As a zero approximation, I assumed that the measurement uncertainties of unblended lines are equal to 20 units of the last given decimal place and twice that for blended or perturbed lines. With these uncertainties, a set of optimized energy levels was obtained with the LOPT code [23]. The resulting level values agreed with those given by Paschen and Campbell [2] with a standard deviation of $0.04 \mathrm{~cm}^{-1}$. However, the too small residuals (i.e., deviations of observed wave numbers from the Ritz values) indicated that the wavenumber uncertainties should be decreased in order to achieve statistical consistency. The latter requirement means that the root of sum of squares (RSS) of the residuals should be approximately equal to degrees of freedom (DF) of the problem (in this case, $\mathrm{DF}=320$ ). With the initial estimate of uncertainties described above, $\mathrm{RSS} / \mathrm{DF}=0.12$, which means that the assumed uncertainties were too large on average. Better estimates can be obtained by analyzing mean values of residuals (observed minus Ritz wave number) separately for each category of lines. Division of the lines into categories is naturally defined by the different observation methods (with the single-prism, three-prism, or grating spectrograph in different orders of diffraction) and by the character of the lines (well-resolved or blended). The finally adopted uncertainty values are listed in Table 1 . They vary from $0.02 \mathrm{~cm}^{-1}$ for a few red lines to $1.0 \mathrm{~cm}^{-1}$ for the blended line at $2249.6 \AA$. With these final uncertainty values, the level optimization procedure yields $\mathrm{RSS} / \mathrm{DF}=1.15$, which indicates a good statistical consistency. Agreement of the optimized levels with the original values from Paschen and Campbell remained the same (on average) after the uncertainty adjustments.

The line list of Paschen and Campbell [2] includes 28 forbidden transitions. One of them is the hyperfine-induced clock transition $5 \mathrm{~s}^{2}{ }^{1} \mathrm{~S}_{0}-5 \mathrm{~s} 5 \mathrm{p}^{3} \mathrm{P}^{\circ}{ }_{0}$ at $2364.686 \AA$, and all the others are electricquadrupole (E2) transitions between opposite-parity levels with the change in the angular momentum $\Delta J=2$. In the absence of external fields, these transitions are highly forbidden. However, an electric field present in the discharge mixes the hyperfine components of the levels having the same total angular momentum $F$ but $J$ values differing by one. For example, an external electric field mixes the $F=15 / 2$ component of the $5 \mathrm{~s} 7 \mathrm{f}^{3} \mathrm{~F}^{\circ}{ }_{4}$ level with the $F=15 / 2$ component of the $5 \mathrm{~s} 7 \mathrm{f}^{3} \mathrm{~F}_{3}^{\circ}$ level, thus enabling the normally forbidden $5 \mathrm{~s} 6 \mathrm{~d}^{3} \mathrm{D}_{2}-5 \mathrm{~s} 7 \mathrm{f}^{3} \mathrm{~F}_{4}^{\circ}$ transition. For such an electric-field induced transition, only a certain part of the hfs components of both levels become allowed. Thus, its observed center of gravity will be shifted from the difference between the centers of gravity of the two levels. This may cause systematic shifts in the level values derived with the level optimization procedure. To avoid such shifts, for the purpose of level optimization I have decreased the weight of these forbidden transitions by a factor of four by increasing their uncertainties with a factor of two. However, the uncertainties given in Table 1 are given without these adjustment factors.

A small inconsistency was found in the tables of Paschen and Campbell [2]. Namely, the $5 \mathrm{~s} 17 \mathrm{~s}^{3} \mathrm{~S}_{1}$ level was given in [2] as $149892.67 \mathrm{~cm}^{-1}$. This level takes part in three observed transitions, $5 \mathrm{~s} 6 \mathrm{p}^{3} \mathrm{P}_{0,1,2}-$ $5 \mathrm{~s} 17 \mathrm{~s}^{3} \mathrm{~S}_{1}$ at $2367.0 \AA, 2377.1 \AA$, and $2410.8 \AA$, all measured with the single-prism spectrograph. The Ritz wave number given in the line list of [2] for the ${ }^{3} \mathrm{P}_{1}-{ }^{3} \mathrm{~S}_{1}$ transition agrees with the listed energy levels. However, the Ritz values given for the other two transitions imply a different value of the $5 \mathrm{~s} 17 \mathrm{~s}{ }^{3} \mathrm{~S}_{1}$ level, $149891.99 \mathrm{~cm}^{-1}$.

\subsection{Measurements of Bhatia [5]}

The thesis of Bhatia [5] was mainly focused on the spectra of doubly and triply ionized indium. However, it also includes new energy-level classifications for about a hundred of In II lines. For another several tens of lines, Bhatia's measurements are the most accurate available. Emission spectra of indium were photographed over the spectral range from $340 \AA$ to $9500 \AA$ using a disruptive electrodeless discharge and a spark in helium. About 4000 lines of indium were measured, and $36 \%$ of these lines were classified as belonging to the In I-V spectra. The spectrum from $340 \AA$ to $2440 \AA$ was obtained using a $3 \mathrm{~m}$ grazing incidence vacuum spectrograph with a 1200 lines $/ \mathrm{mm}$ grating giving a reciprocal dispersion of $2.775 \AA / \mathrm{mm}$. The grating was blazed for $1300 \AA$. In the region $2300 \AA$ to $9500 \AA$, a prism spectrograph was used. Different types of photographic plates were used in three spectral ranges: $340 \AA$ to $3000 \AA$, 
$3000 \AA$ to $6500 \AA$, and $4500 \AA$ to $9500 \AA$. For the wavelength scale calibration in the vacuum range, $\mathrm{C}, \mathrm{N}$, and O standards were used below $1760 \AA$ and Si standards between $1930 \AA$ and $2297 \AA$. For the prism spectra, iron and neon standards were used.

Bhatia noted that the prism measurements have uncertainties greater than $\pm 0.05 \AA$. This corresponds to a wavenumber uncertainty of $\pm 0.8 \mathrm{~cm}^{-1}$ at $2440 \AA$ and $\pm 0.05 \mathrm{~cm}^{-1}$ at $9600 \AA$. For the grating measurements, I estimated uncertainties by comparing the measured wavelengths with much more accurate Ritz values derived from the measurements described in the previous sections. In the region below $1900 \AA$, the statistical uncertainty of well-resolved lines is $\pm 0.03 \AA$; between $1900 \AA$ and $2440 \AA$, it is $\pm 0.09 \AA$; and above $2440 \AA$, it is $\pm 0.13 \AA$.

All newly classified or re-measured In II lines in Bhatia's thesis correspond to transitions between known levels determined from other, more accurate measurements. Therefore, these lines were not included in the level optimization procedure. However, for the sake of completeness, I include them in the line list and give their original observed wavelengths. They were found to contain noticeable systematic shifts, which are responsible for the difference between the values of the correction to the energy levels of Paschen and Campbell derived by different authors $[6,11]$. These systematic shifts were estimated by fitting deviations of Bhatia's original wavelengths from the Ritz values with cubic polynomials. This was done separately for the prism and grating measurements. For the grating measurements, systematic shifts vary from $-0.02 \AA$ at $2430 \AA$ to $+0.01 \AA$ at $1900 \AA,-0.01 \AA$ at $1000 \AA$, and $+0.005 \AA$ at $680 \AA$. For the prism measurements, they vary from $-0.44 \AA$ at $5123 \AA$ to $+0.10 \AA$ at $2460 \AA$.

In Table 1, the observed wavelengths selected from Bhatia [5] are given with two uncertainty values in parentheses after the value. The first uncertainty is a statistical one, and the second is a systematic one.

\subsection{Measurements of Lang and Sawyer [3]}

Lang and Sawyer [3] observed the In II spectrum emitted by a hollow cathode discharge in helium or in neon. As cathodes, they used a carbon tube with indium placed inside or a tungsten tube coated with indium. The spectrum between $500 \AA$ and $8000 \AA$ was photographed with a $1 \mathrm{~m}$ vacuum grating spectrograph and with two prism spectrographs. Lang and Sawyer did not specify their measurement uncertainties, but indicated that the higher terms deduced from the vacuum ultraviolet measurements should be accurate to less than $5 \mathrm{~cm}^{-1}$, which corresponds to $\pm 0.05 \AA$ at $1000 \AA$.

Lang and Sawyer listed 144 observed and classified lines of In II between $680 \AA$ and $7183 \AA$. All their measurements above $3273 \AA$ have been superseded by other observers. However, in the shorter wavelength region there remain 18 lines for which their observations are the only available ones. All of the levels associated with these lines are accurately established by other observations, so I did not use the lines from [3] in the level optimization procedure. Nevertheless, I include them in Table I with their original wavelengths. They were found to contain significant systematic shifts. These shifts were estimated by fitting deviations of the original wavelengths from the Ritz values with a cubic polynomial. The systematic shifts vary from $-0.25 \AA$ at $680 \AA$ to $-0.07 \AA$ at $1586 \AA$ and $-0.14 \AA$ at $3273 \AA$, while the average statistical uncertainty is $\pm 0.11 \AA$ below $2300 \AA$ and $\pm 0.09 \AA$ above that.

Similar to Bhatia's measurements, the observed wavelengths selected from Lang and Sawyer [3] are given in Table 1 with two uncertainties, statistical and systematic, given in parentheses after the value.

Five lines listed by Lang and Sawyer [3] at $1133.15 \AA, 1625.36 \AA, 1681.72 \AA, 2446.02 \AA$, and $3274.11 \AA$ do not match any combination between the known energy levels. For the line at $1133.15 \AA$, I found that Lang and Sawyer made an error in conversion from the correctly given wavenumber $87249 \mathrm{~cm}^{-1}$. The correct wavelength is $1146.14 \AA$. The line at 1625.36 was classified by Bhatia [5] as belonging to In III. For the line at $3274.11 \AA$, the wave number was misprinted in Lang and Sawyer as 30534 , while the correct value is $30543 \mathrm{~cm}^{-1}$. Thus, the correct wavelength is $3273.13 \AA$. Paschen and Campbell [2] noted that this line was expected to occur in their spectra, but they could not detect it. The origins of the other two lines are unknown. Thus, they are omitted from Table 1. 


\subsection{Measurements of Wagatsuma [15]}

Wagatsuma [15] observed emission spectra of several elements, including indium, from Grimm glow discharge plasmas with argon, neon, and mixtures of argon with helium and neon with helium as buffer gases. The main purpose was to investigate relative intensities of spectral lines in different buffer gases. The Grimm-type discharge features a hollow anode, and a plain sample is placed close to the anode opening. A Fastie-Ebert mounting spectrograph with a focal length of 3.4 m equipped with a photomultiplier detector was employed to measure the spectra in the wavelength region from $2300 \AA$ to $8000 \AA$. The grating had 1200 lines $/ \mathrm{mm}$ and was blazed for $3000 \AA$. Emission spectra in the shorter wavelength region, $1600 \AA$ to $2450 \AA$, were recorded on a $2.0 \mathrm{~m}$ Eagle-mounting vacuum spectrometer with a 1200 lines $/ \mathrm{mm}$ grating blazed at $1700 \AA$, equipped with a $\mathrm{CaF}_{2}$ window and a photomultiplier tube.

Wagatsuma reported intensity measurements for 43 lines of In II between $1571 \AA$ and $6116 \AA$. He did not give his measured wavelengths; instead, he listed the Ritz wavelengths calculated from the energy levels given by Moore [4]. Most of the lines were observed and classified elsewhere. However, two lines were newly classified. These are the lines at $1657.43 \AA\left(5 \mathrm{~s} 5 \mathrm{p}^{1} \mathrm{P}^{\circ}{ }_{1}-5 \mathrm{~s} 7 \mathrm{~s}{ }^{1} \mathrm{~S}_{0}\right)$ and $3842.92 \AA\left(5 \mathrm{p}^{2}{ }^{1} \mathrm{D}_{2}-\right.$ $5 \mathrm{~s} 4 \mathrm{f}^{3} \mathrm{~F}^{\circ}{ }_{2}$ ). Since no wavelength measurements are available, I give the Ritz wavelengths for these two lines in Table 1.

\section{Optimized Energy Levels and the Ionization Limit}

After the wavelength measurement uncertainties have been assessed, the level optimization is a straightforward procedure. For that I used the least-squares level optimization code LOPT [23]. As noted above, only the measurements of Karlsson and Litzén [11], Larkins and Hannaford [8], von Zanthier et al. [10], and Paschen and Campbell [2] were included in the level optimization procedure, which makes a total of 495 lines. Although high-precision measurements constitute only about $10 \%$ of all included lines, they have a dramatic effect on the accuracy of the derived excitation energies, decreasing the uncertainties from a few reciprocal centimeters for the level list of Paschen and Campbell [2] to $0.2 \mathrm{~cm}^{-1}$ on average. More than half of the levels have uncertainties below $0.1 \mathrm{~cm}^{-1}$, and 75 of them are accurate to better than $\pm 0.05 \mathrm{~cm}^{-1}$.

The average shift of the newly optimized levels above $63000 \mathrm{~cm}^{-1}$ from those given by Paschen and Campbell [2] is $4.88 \mathrm{~cm}^{-1}$ with a standard deviation of $0.18 \mathrm{~cm}^{-1}$. For two levels from the list of Paschen and Campbell, $5 \mathrm{~s} 9 \mathrm{p}^{3} \mathrm{P}_{2}^{\circ}$ and $5 \mathrm{~s} 11 \mathrm{p}^{3} \mathrm{P}^{\circ}{ }_{1}$, there are no observed lines in their line list. Transitions from these levels were observed by Bhatia [5] (one from $5 \mathrm{~s} 9 \mathrm{p}^{3} \mathrm{P}^{\circ}{ }_{2}$ and four from $5 \mathrm{~s} 11 \mathrm{p}^{3} \mathrm{P}_{1}^{\circ}$ ). Their measurement uncertainties are between $1.6 \mathrm{~cm}^{-1}$ and $1.9 \mathrm{~cm}^{-1}$, which is much greater than uncertainties in the measurements of Paschen and Campbell. I assumed that those lines were actually observed by Paschen and Campbell but omitted from their line list. Therefore, the values given for these levels in Table 2 are derived from those of Paschen and Campbell by adding the average shift mentioned above.

As can be seen in Table 2, there are several Rydberg series accurately measured up to high values of principal quantum number $n$, such as $5 \mathrm{~s} n \mathrm{~d}^{3} \mathrm{D}_{3}(n=5-19), 5 \mathrm{~s} n \mathrm{~d}^{3} \mathrm{D}_{2}$ and ${ }^{3} \mathrm{D}_{1}(n=5-18), 5 \mathrm{~s} n \mathrm{~s}^{3} \mathrm{~S}_{1}(n=5-$ 17), 5sng $(n=5-14)$, and $5 \mathrm{~s} n \mathrm{~h}(n=8-14)$. These series are unperturbed and thus can be used to determine the ionization limit. I used computer codes written by Sansonetti [29] to fit Ritz-type quantum-defect formulas for the $5 \mathrm{~s} n \mathrm{~s}$ and $5 \mathrm{~s} n$ d series and the polarization formula for the $5 \mathrm{~s} n \mathrm{~g}$ and $5 \mathrm{~s} n \mathrm{~h}$ series. Thus, I have obtained five values for the ionization limit derived with different methods, one with the polarization formula and four with quantum-defect formulas. The weighted average of these five values is $152200.10 \mathrm{~cm}^{-1}$ with $\pm 0.22 \mathrm{~cm}^{-1}$ adopted as a conservative estimate of uncertainty.

From the same or similar series formulas, several unobserved levels, such as $5 \mathrm{~s} 6 \mathrm{~h}, 5 \mathrm{~s} 7 \mathrm{~h}, 5 \mathrm{~s} 15 \mathrm{~d}^{3} \mathrm{D}_{1}$, $5 \mathrm{~s} 19 \mathrm{~d}^{3} \mathrm{D}_{1}, 5 \mathrm{~s} 19 \mathrm{~d}^{3} \mathrm{D}_{2}, 5 \mathrm{~s} 18 \mathrm{~s}^{3} \mathrm{~S}_{1}, 5 \mathrm{~s} 19 \mathrm{~s}^{3} \mathrm{~S}_{1}$, and $5 \mathrm{~s} n \mathrm{~s}^{1} \mathrm{~S}_{0}(n=17-19)$, could be accurately determined. For two levels, $5 \mathrm{~s} 12 \mathrm{p}^{1} \mathrm{P}^{\circ}{ }_{1}$ and $5 \mathrm{~s} 17 \mathrm{~d}^{3} \mathrm{D}_{3}$, the values derived from the series formulas are significantly more accurate than the measured ones. These interpolated or extrapolated values are included in Table 2.

The only two high-precision energy values in Table 2, i.e., those for the $5 \mathrm{~s} 5 \mathrm{p}^{3} \mathrm{P}_{0}^{\circ}$ and $5 \mathrm{~s} 5 \mathrm{p}^{3} \mathrm{P}^{\circ}{ }_{1}$ levels, are pertinent to the isotope ${ }^{115} \mathrm{In}$, while the rest of the level values were determined from wavelengths of lines observed in a natural mixture of isotopes. The only available experimental measurement of the isotope 
Table 2. Optimized energy levels of In II

\begin{tabular}{|c|c|c|c|c|c|c|c|c|}
\hline Configuration & Term & $J$ & $\operatorname{Level}^{\mathrm{a}}\left(\mathrm{cm}^{-1}\right)$ & \multicolumn{4}{|c|}{ Leading percentages ${ }^{\mathrm{b}}$} & Comments $^{c}$ \\
\hline $5 s^{2}$ & ${ }^{1} \mathrm{~S}$ & 0 & 0.000000000 & 98 & & & & \\
\hline $5 \mathrm{~s} 5 \mathrm{p}$ & ${ }^{3} \mathrm{P}^{\circ}$ & 0 & $42275.995245348(8)$ & 100 & & & & \\
\hline $5 \mathrm{~s} 5 \mathrm{p}$ & ${ }^{3} \mathrm{P}^{\circ}$ & 1 & $43350.5817(15)$ & 99 & & & & \\
\hline $5 \mathrm{~s} 5 \mathrm{p}$ & ${ }^{3} \mathrm{P}^{\circ}$ & 2 & $45829.256(6)$ & 100 & & & & \\
\hline $5 \mathrm{~s} 5 \mathrm{p}$ & ${ }^{1} \mathrm{P}^{\circ}$ & 1 & $63038.546(10)$ & 98 & & & & \\
\hline $5 s 6 s$ & ${ }^{3} \mathrm{~S}$ & 1 & $93923.884(5)$ & 100 & & & & \\
\hline $5 \mathrm{~s} 6 \mathrm{~s}$ & ${ }^{1} \mathrm{~S}$ & 0 & $97030.212(12)$ & 99 & & & & \\
\hline $5 \mathrm{~s} 5 \mathrm{~d}$ & ${ }^{1} \mathrm{D}$ & 2 & $97628.436(9)$ & 67 & 31 & $5 p^{2}$ & ${ }^{1} \mathrm{D}$ & \\
\hline $5 p^{2}$ & ${ }^{3} \mathrm{P}$ & 0 & $101608.06(20)$ & 97 & & & & \\
\hline $5 \mathrm{~s} 5 \mathrm{~d}$ & ${ }^{3} \mathrm{D}$ & 1 & $102088.72(4)$ & 100 & & & & \\
\hline $5 \mathrm{~s} 5 \mathrm{~d}$ & ${ }^{3} \mathrm{D}$ & 2 & $102174.69(3)$ & 100 & & & & \\
\hline $5 \mathrm{~s} 5 \mathrm{~d}$ & ${ }^{3} \mathrm{D}$ & 3 & $102308.397(20)$ & 100 & & & & \\
\hline $5 p^{2}$ & ${ }^{3} \mathrm{P}$ & 1 & $103249.39(19)$ & 100 & & & & \\
\hline $5 p^{2}$ & ${ }^{3} \mathrm{P}$ & 2 & $105565.283(12)$ & 96 & & & & \\
\hline $5 \mathrm{~s} 6 \mathrm{p}$ & ${ }^{3} \mathrm{P}^{\circ}$ & 0 & $107662.707(6)$ & 100 & & & & \\
\hline $5 s 6 p$ & ${ }^{3} \mathrm{P}^{\circ}$ & 1 & $107841.992(6)$ & 96 & 4 & & ${ }^{1} \mathrm{P}^{\circ}$ & \\
\hline $5 s 6 p$ & ${ }^{3} \mathrm{P}^{\circ}$ & 2 & $108430.337(6)$ & 100 & & & & \\
\hline $5 s 6 p$ & ${ }^{1} \mathrm{P}^{\circ}$ & 1 & $109780.221(8)$ & 94 & 4 & & ${ }^{3} \mathrm{P}^{\circ}$ & \\
\hline $5 p^{2}$ & ${ }^{1} \mathrm{D}$ & 2 & $113884.919(11)$ & 58 & 27 & $5 \mathrm{~s} 5 \mathrm{~d}$ & ${ }^{1} \mathrm{D}$ & \\
\hline $5 p^{2}$ & ${ }^{1} \mathrm{~S}$ & 0 & $121289.53(3)$ & 56 & 42 & $5 \mathrm{~s} 7 \mathrm{~s}$ & ${ }^{1} \mathrm{~S}$ & \\
\hline $5 \mathrm{~s} 7 \mathrm{~s}$ & ${ }^{3} \mathrm{~S}$ & 1 & $121442.541(12)$ & 100 & & & & \\
\hline $5 \mathrm{~s} 7 \mathrm{~s}$ & ${ }^{1} \mathrm{~S}$ & 0 & $123372.848(18)$ & 57 & 39 & $5 p^{2}$ & ${ }^{1} \mathrm{~S}$ & \\
\hline $5 s 4 f$ & ${ }^{3} \mathrm{~F}^{\circ}$ & 2 & $123642.95(3)$ & 100 & & & & \\
\hline $5 s 4 f$ & ${ }^{3} \mathrm{~F}^{\circ}$ & 3 & $123648.096(24)$ & 100 & & & & \\
\hline $5 s 4 f$ & ${ }^{3} \mathrm{~F}^{\circ}$ & 4 & $123664.85(3)$ & 100 & & & & \\
\hline $5 s 4 f$ & ${ }^{1} \mathrm{~F}^{\circ}$ & 3 & $123699.170(10)$ & 100 & & & & \\
\hline $5 \mathrm{~s} 6 \mathrm{~d}$ & ${ }^{3} \mathrm{D}$ & 1 & $124742.729(6)$ & 100 & & & & \\
\hline $5 \mathrm{~s} 6 \mathrm{~d}$ & ${ }^{3} \mathrm{D}$ & 2 & $124776.714(6)$ & 100 & & & & \\
\hline $5 \mathrm{~s} 6 \mathrm{~d}$ & ${ }^{3} \mathrm{D}$ & 3 & $124830.176(8)$ & 100 & & & & \\
\hline $5 s 6 d$ & ${ }^{1} \mathrm{D}$ & 2 & $126670.945(8)$ & 88 & 7 & $5 p^{2}$ & ${ }^{1} \mathrm{D}$ & \\
\hline $5 \mathrm{~s} 7 \mathrm{p}$ & ${ }^{3} \mathrm{P}^{\circ}$ & 0 & $126932.875(22)$ & 100 & & & & \\
\hline $5 \mathrm{~s} 7 \mathrm{p}$ & ${ }^{3} \mathrm{P}^{\circ}$ & 1 & $126994.890(11)$ & 91 & 9 & & ${ }^{1} \mathrm{P}^{\circ}$ & \\
\hline $5 \mathrm{~s} 7 \mathrm{p}$ & ${ }^{3} \mathrm{P}^{\circ}$ & 2 & $127254.067(7)$ & 100 & & & & \\
\hline $5 \mathrm{~s} 7 \mathrm{p}$ & ${ }^{1} \mathrm{P}^{\circ}$ & 1 & $127573.319(10)$ & 90 & 9 & & ${ }^{3} \mathrm{P}^{\circ}$ & \\
\hline $5 \mathrm{~s} 8 \mathrm{~s}$ & ${ }^{3} \mathrm{~S}$ & 1 & $133072.511(7)$ & 100 & & & & \\
\hline $5 \mathrm{~s} 8 \mathrm{~s}$ & ${ }^{1} \mathrm{~S}$ & 0 & $133554.382(14)$ & 98 & & & & \\
\hline $5 \mathrm{~s} 5 \mathrm{f}$ & ${ }^{3} \mathrm{~F}^{\circ}$ & 2 & $133940.74(4)$ & 100 & & & & \\
\hline $5 \mathrm{~s} 5 \mathrm{f}$ & ${ }^{3} \mathrm{~F}^{\circ}$ & 3 & $133944.72(6)$ & 100 & & & & \\
\hline $5 s 5 f$ & ${ }^{3} \mathrm{~F}^{\circ}$ & 4 & $133960.87(3)$ & 100 & & & & \\
\hline $5 s 5 f$ & ${ }^{1} \mathrm{~F}^{\circ}$ & 3 & $133984.85(6)$ & 100 & & & & \\
\hline $5 \mathrm{~s}_{1 / 2, F=4} 5 \mathrm{~g}$ & & & $134512.23(6)$ & & & & & \\
\hline $5 \mathrm{~s}_{1 / 2, F=5} 5 \mathrm{~g}$ & & & $134516.14(6)$ & & & & & \\
\hline $5 \mathrm{~s} 7 \mathrm{~d}$ & ${ }^{3} \mathrm{D}$ & 1 & $134726.488(10)$ & 100 & & & & \\
\hline $5 \mathrm{~s} 7 \mathrm{~d}$ & ${ }^{3} \mathrm{D}$ & 2 & $134744.019(9)$ & 100 & & & & \\
\hline
\end{tabular}




\begin{tabular}{|c|c|c|c|c|c|c|c|}
\hline Configuration & Term & $J$ & Level $^{\mathrm{a}}\left(\mathrm{cm}^{-1}\right)$ & \multicolumn{3}{|c|}{ Leading percentages $^{\mathrm{b}}$} & Comments ${ }^{c}$ \\
\hline $5 \mathrm{~s} 7 \mathrm{~d}$ & ${ }^{3} \mathrm{D}$ & 3 & $134771.897(9)$ & 100 & & & \\
\hline $5 \mathrm{~s} 7 \mathrm{~d}$ & ${ }^{1} \mathrm{D}$ & 2 & $135400.325(10)$ & 95 & & & \\
\hline $5 \mathrm{~s} 8 \mathrm{p}$ & ${ }^{3} \mathrm{P}^{\circ}$ & 0 & {$[135823(11)]$} & 100 & & & LSF \\
\hline $5 s 8 p$ & ${ }^{3} \mathrm{P}^{\circ}$ & 1 & $135861.62(5)$ & 86 & 14 & ${ }^{1} \mathrm{P}^{\circ}$ & \\
\hline $5 \mathrm{~s} 8 \mathrm{p}$ & ${ }^{3} \mathrm{P}^{\circ}$ & 2 & $135999.37(23)$ & 100 & & & \\
\hline $5 \mathrm{~s} 8 \mathrm{p}$ & ${ }^{1} \mathrm{P}^{\circ}$ & 1 & $136096.93(9)$ & 85 & 14 & ${ }^{3} \mathrm{P}^{\circ}$ & \\
\hline $5 \mathrm{~s} 9 \mathrm{~s}$ & ${ }^{3} \mathrm{~S}$ & 1 & $139137.055(23)$ & 100 & & & \\
\hline $5 \mathrm{~s} 9 \mathrm{~s}$ & ${ }^{1} \mathrm{~S}$ & 0 & $139387.30(5)$ & 99 & & & \\
\hline $5 s 6 f$ & ${ }^{3} \mathrm{~F}^{\circ}$ & 2 & $139549.42(10)$ & 100 & & & \\
\hline $5 s 6 f$ & ${ }^{3} \mathrm{~F}^{\circ}$ & 3 & $139552.75(7)$ & 100 & & & \\
\hline $5 s 6 f$ & ${ }^{3} \mathrm{~F}^{\circ}$ & 4 & $139567.37(14)$ & 100 & & & \\
\hline $5 s 6 f$ & ${ }^{1} \mathrm{~F}^{\circ}$ & 3 & $139586.14(8)$ & 100 & & & \\
\hline $5 \mathrm{~s}_{1 / 2, F=4} 6 \mathrm{~g}$ & & & $139921.76(7)$ & & & & \\
\hline $5 \mathrm{~s}_{1 / 2, F=5} 6 \mathrm{~g}$ & & & $139925.47(7)$ & & & & \\
\hline $5 \mathrm{~s}_{1 / 2, F=4} 6 \mathrm{~h}$ & & & {$[139980(3)]$} & & & & POLAR \\
\hline $5 \mathrm{~s}_{1 / 2, F=5} 6 \mathrm{~h}$ & & & [139984(3)] & & & & POLAR \\
\hline $5 \mathrm{~s} 8 \mathrm{~d}$ & ${ }^{3} \mathrm{D}$ & 1 & $140082.23(3)$ & 100 & & & \\
\hline $5 \mathrm{~s} 8 \mathrm{~d}$ & ${ }^{3} \mathrm{D}$ & 2 & $140092.64(4)$ & 100 & & & \\
\hline $5 \mathrm{~s} 8 \mathrm{~d}$ & ${ }^{3} \mathrm{D}$ & 3 & $140109.05(5)$ & 100 & & & \\
\hline $5 \mathrm{~s} 8 \mathrm{~d}$ & ${ }^{1} \mathrm{D}$ & 2 & $140408.36(5)$ & 97 & & & \\
\hline $5 s 9 p$ & ${ }^{3} \mathrm{P}^{\circ}$ & 0 & {$[140716(17)]$} & 100 & & & LSF \\
\hline $5 \mathrm{~s} 9 \mathrm{p}$ & ${ }^{3} \mathrm{P}^{\circ}$ & 1 & $140734.59(5)$ & 85 & 15 & ${ }^{1} \mathrm{P}^{\circ}$ & \\
\hline $5 \mathrm{~s} 9 \mathrm{p}$ & ${ }^{3} \mathrm{P}^{\circ}$ & 2 & $140822.48(18)$ & 100 & & & \\
\hline $5 \mathrm{~s} 9 \mathrm{p}$ & ${ }^{1} \mathrm{P}^{\circ}$ & 1 & $140845.00(4)$ & 84 & 15 & ${ }^{3} \mathrm{P}^{\circ}$ & \\
\hline $5 \mathrm{~s} 10 \mathrm{~s}$ & ${ }^{3} \mathrm{~S}$ & 1 & $142708.324(25)$ & 100 & & & \\
\hline $5 \mathrm{~s} 10 \mathrm{~s}$ & ${ }^{1} \mathrm{~S}$ & 0 & $142856.86(3)$ & 99 & & & \\
\hline $5 \mathrm{~s} 7 \mathrm{f}$ & ${ }^{3} \mathrm{~F}^{\circ}$ & 2 & $142927.81(3)$ & 100 & & & \\
\hline $5 \mathrm{~s} 7 \mathrm{f}$ & ${ }^{3} \mathrm{~F}^{\circ}$ & 3 & $142930.22(5)$ & 100 & & & \\
\hline $5 \mathrm{~s} 7 \mathrm{f}$ & ${ }^{3} \mathrm{~F}^{\circ}$ & 4 & $142943.23(16)$ & 100 & & & \\
\hline $5 \mathrm{~s} 7 \mathrm{f}$ & ${ }^{1} \mathrm{~F}^{\circ}$ & 3 & $142959.77(9)$ & 100 & & & \\
\hline $5 \mathrm{~s}_{1 / 2, F=4} 7 \mathrm{~g}$ & & & $143183.87(5)$ & & & & \\
\hline $5 \mathrm{~s}_{1 / 2, F=5} 7 \mathrm{~g}$ & & & $143187.43(5)$ & & & & \\
\hline $5 \mathrm{~s}_{1 / 2, F=4} 7 \mathrm{~h}$ & & & {$[143222.8(10)]$} & & & & POLAR \\
\hline $5 \mathrm{~s}_{1 / 2, F=5} 7 \mathrm{~h}$ & & & {$[143226.3(10)]$} & & & & POLAR \\
\hline $5 \mathrm{~s} 9 \mathrm{~d}$ & ${ }^{3} \mathrm{D}$ & 1 & $143298.47(4)$ & 100 & & & \\
\hline $5 \mathrm{~s} 9 \mathrm{~d}$ & ${ }^{3} \mathrm{D}$ & 2 & $143305.21(3)$ & 100 & & & \\
\hline $5 \mathrm{~s} 9 \mathrm{~d}$ & ${ }^{3} \mathrm{D}$ & 3 & $143315.78(14)$ & 100 & & & \\
\hline $5 \mathrm{~s} 9 \mathrm{~d}$ & ${ }^{1} \mathrm{D}$ & 2 & $143483.89(7)$ & 97 & & & \\
\hline $5 \mathrm{~s} 10 \mathrm{p}$ & ${ }^{3} \mathrm{P}^{\circ}$ & 0 & {$[143676(17)]$} & 100 & & & LSF \\
\hline $5 \mathrm{~s} 10 \mathrm{p}$ & ${ }^{3} \mathrm{P}^{\circ}$ & 1 & $143706.54(8)$ & 88 & & ${ }^{1} \mathrm{P}^{\circ}$ & \\
\hline $5 \mathrm{~s} 10 \mathrm{p}$ & ${ }^{3} \mathrm{P}^{\circ}$ & 2 & $143740.9(3)$ & 100 & & & \\
\hline $5 \mathrm{~s} 10 \mathrm{p}$ & ${ }^{1} \mathrm{P}^{\circ}$ & 1 & $143763.10(8)$ & 87 & & ${ }^{3} \mathrm{P}^{\circ}$ & \\
\hline $5 \mathrm{~s} 11 \mathrm{~s}$ & ${ }^{3} \mathrm{~S}$ & 1 & 144989.99(4) & 100 & & & \\
\hline $5 \mathrm{~s} 11 \mathrm{~s}$ & ${ }^{1} \mathrm{~S}$ & 0 & $145086.25(4)$ & 99 & & & \\
\hline $5 \mathrm{~s} 8 \mathrm{f}$ & ${ }^{3} \mathrm{~F}^{\circ}$ & 2 & $145115.79(15)$ & 100 & & & \\
\hline
\end{tabular}




\begin{tabular}{|c|c|c|c|c|c|}
\hline Configuration & Term & $J$ & $\operatorname{Level}^{\mathrm{a}}\left(\mathrm{cm}^{-1}\right)$ & Leading percentages $^{\mathrm{b}}$ & Comments $^{c}$ \\
\hline $5 \mathrm{~s} 8 \mathrm{f}$ & ${ }^{3} \mathrm{~F}^{\circ}$ & 3 & $145117.59(11)$ & 100 & \\
\hline $5 \mathrm{~s} 8 \mathrm{f}$ & ${ }^{3} \mathrm{~F}^{\circ}$ & 4 & $145129.44(20)$ & 100 & \\
\hline $5 \mathrm{~s} 8 \mathrm{f}$ & ${ }^{1} \mathrm{~F}^{\circ}$ & 3 & $145144.45(11)$ & 100 & \\
\hline $5 \mathrm{~s}_{1 / 2, F=4} 8 \mathrm{~g}$ & & & $145300.34(4)$ & & \\
\hline $5 \mathrm{~s}_{1 / 2, F=5} 8 \mathrm{~g}$ & & & $145303.91(4)$ & & \\
\hline $5 \mathrm{~s}_{1 / 2, F=4} 8 \mathrm{~h}$ & & & $145326.55(21)$ & & \\
\hline $5 \mathrm{~s}_{1 / 2, F=5} 8 \mathrm{~h}$ & & & $145330.18(21)$ & & \\
\hline $5 \mathrm{~s} 10 \mathrm{~d}$ & ${ }^{3} \mathrm{D}$ & 1 & $145382.99(4)$ & 100 & \\
\hline $5 \mathrm{~s} 10 \mathrm{~d}$ & ${ }^{3} \mathrm{D}$ & 2 & $145387.70(6)$ & 100 & \\
\hline $5 \mathrm{~s} 10 \mathrm{~d}$ & ${ }^{3} \mathrm{D}$ & 3 & $145394.94(4)$ & 100 & \\
\hline $5 \mathrm{~s} 10 \mathrm{~d}$ & ${ }^{1} \mathrm{D}$ & 2 & $145499.56(7)$ & 98 & \\
\hline $5 \mathrm{~s} 11 \mathrm{p}$ & ${ }^{3} \mathrm{P}^{\circ}$ & 1 & $145655.81(18)$ & & \\
\hline $5 \mathrm{~s} 11 \mathrm{p}$ & ${ }^{1} \mathrm{P}^{\circ}$ & 1 & $145683.72(14)$ & & \\
\hline $5 \mathrm{~s} 12 \mathrm{~s}$ & ${ }^{3} \mathrm{~S}$ & 1 & $146537.16(4)$ & 100 & \\
\hline $5 \mathrm{~s} 12 \mathrm{~s}$ & ${ }^{1} \mathrm{~S}$ & 0 & $146602.97(5)$ & 99 & \\
\hline $5 \mathrm{~s} 9 \mathrm{f}$ & ${ }^{3} \mathrm{~F}^{\circ}$ & 3 & $146612.28(11)$ & 100 & \\
\hline $5 \mathrm{~s} 9 \mathrm{f}$ & ${ }^{3} \mathrm{~F}^{\circ}$ & 2 & $146614.22(4)$ & 100 & \\
\hline $5 \mathrm{~s} 9 \mathrm{f}$ & ${ }^{3} \mathrm{~F}^{\circ}$ & 4 & $146624.75(15)$ & 100 & \\
\hline $5 \mathrm{~s} 9 \mathrm{f}$ & ${ }^{1} \mathrm{~F}^{\circ}$ & 3 & $146638.37(13)$ & 100 & \\
\hline $5 \mathrm{~s}_{1 / 2, F=4} 9 \mathrm{~g}$ & & & $146750.53(5)$ & & \\
\hline $5 \mathrm{~s}_{1 / 2, F=5} 9 \mathrm{~g}$ & & & 146754.16(4) & & \\
\hline $5 \mathrm{~s}_{1 / 2, F=4} 9 \mathrm{~h}$ & & & $146769.69(15)$ & & \\
\hline $5 \mathrm{~s}_{1 / 2, F=5} 9 \mathrm{~h}$ & & & $146773.23(15)$ & & \\
\hline $5 \mathrm{~s} 11 \mathrm{~d}$ & ${ }^{3} \mathrm{D}$ & 1 & $146812.50(9)$ & & \\
\hline $5 \mathrm{~s} 11 \mathrm{~d}$ & ${ }^{3} \mathrm{D}$ & 2 & $146815.24(9)$ & & \\
\hline $5 \mathrm{~s} 11 \mathrm{~d}$ & ${ }^{3} \mathrm{D}$ & 3 & $146820.60(14)$ & & \\
\hline $5 \mathrm{~s} 11 \mathrm{~d}$ & ${ }^{1} \mathrm{D}$ & 2 & $146890.14(11)$ & & \\
\hline $5 \mathrm{~s} 12 \mathrm{p}$ & ${ }^{1} \mathrm{P}^{\circ}$ & 1 & {$[147016(10)]$} & & RITZPL \\
\hline $5 \mathrm{~s} 13 \mathrm{~s}$ & ${ }^{3} \mathrm{~S}$ & 1 & $147634.61(6)$ & 100 & \\
\hline $5 \mathrm{~s} 10 \mathrm{f}$ & ${ }^{3} \mathrm{~F}^{\circ}$ & 3 & $147680.47(13)$ & 100 & \\
\hline $5 \mathrm{~s} 13 \mathrm{~s}$ & ${ }^{1} \mathrm{~S}$ & 0 & $147681.43(7)$ & 99 & \\
\hline $5 \mathrm{~s} 10 \mathrm{f}$ & ${ }^{3} \mathrm{~F}^{\circ}$ & 2 & $147682.61(14)$ & 100 & \\
\hline $5 \mathrm{~s} 10 \mathrm{f}$ & ${ }^{3} \mathrm{~F}^{\circ}$ & 4 & $147691.91(15)$ & 100 & \\
\hline $5 \mathrm{~s} 10 \mathrm{f}$ & ${ }^{1} \mathrm{~F}^{\circ}$ & 3 & $147704.35(16)$ & 100 & \\
\hline $5 \mathrm{~s}_{1 / 2, F=4} 10 \mathrm{~g}$ & & & $147787.35(5)$ & & \\
\hline $5 \mathrm{~s}_{1 / 2, F=5} 10 \mathrm{~g}$ & & & $147790.88(6)$ & & \\
\hline $5 \mathrm{~s}_{1 / 2, F=4} 10 \mathrm{~h}$ & & & $147801.67(20)$ & & \\
\hline $5 \mathrm{~s}_{1 / 2, F=5} 10 \mathrm{~h}$ & & & $147804.99(20)$ & & \\
\hline $5 \mathrm{~s} 12 \mathrm{~d}$ & ${ }^{3} \mathrm{D}$ & 1 & $147833.52(8)$ & & \\
\hline $5 \mathrm{~s} 12 \mathrm{~d}$ & ${ }^{3} \mathrm{D}$ & 2 & $147836.29(17)$ & & \\
\hline $5 \mathrm{~s} 12 \mathrm{~d}$ & ${ }^{3} \mathrm{D}$ & 3 & $147840.27(19)$ & & \\
\hline $5 \mathrm{~s} 12 \mathrm{~d}$ & ${ }^{1} \mathrm{D}$ & 2 & $147888.97(15)$ & & \\
\hline $5 \mathrm{~s} 14 \mathrm{~s}$ & ${ }^{3} \mathrm{~S}$ & 1 & $148440.88(7)$ & 100 & \\
\hline $5 \mathrm{~s} 11 \mathrm{f}$ & ${ }^{3} \mathrm{~F}^{\circ}$ & 3 & $148468.39(14)$ & & \\
\hline $5 \mathrm{~s} 11 \mathrm{f}$ & ${ }^{3} \mathrm{~F}^{\circ}$ & 2 & $148472.06(14)$ & & \\
\hline
\end{tabular}




\begin{tabular}{|c|c|c|c|c|c|}
\hline Configuration & Term & $J$ & Level $^{\mathrm{a}}\left(\mathrm{cm}^{-1}\right)$ & Leading percentages $^{\mathrm{b}}$ & Comments $^{\mathrm{c}}$ \\
\hline $5 \mathrm{~s} 14 \mathrm{~s}$ & ${ }^{1} \mathrm{~S}$ & 0 & {$[148475.56(22)]$} & 99 & RITZPL \\
\hline $5 \mathrm{~s} 11 \mathrm{f}$ & ${ }^{3} \mathrm{~F}^{\circ}$ & 4 & $148479.98(15)$ & & \\
\hline $5 \mathrm{~s} 11 \mathrm{f}$ & ${ }^{1} \mathrm{~F}^{\circ}$ & 3 & $148491.51(14)$ & & \\
\hline $5 \mathrm{~s}_{1 / 2, F=4} 11 \mathrm{~g}$ & & & $148554.36(7)$ & & \\
\hline $5 \mathrm{~s}_{1 / 2, F=5} 11 \mathrm{~g}$ & & & $148557.89(8)$ & & \\
\hline $5 \mathrm{~s}_{1 / 2, F=4} 11 \mathrm{~h}$ & & & $148565.16(21)$ & & \\
\hline $5 \mathrm{~s}_{1 / 2, F=5} 11 \mathrm{~h}$ & & & $148568.78(21)$ & & \\
\hline $5 \mathrm{~s} 13 \mathrm{~d}$ & ${ }^{3} \mathrm{D}$ & 1 & $148589.72(20)$ & & \\
\hline $5 \mathrm{~s} 13 \mathrm{~d}$ & ${ }^{3} \mathrm{D}$ & 2 & $148592.29(18)$ & & \\
\hline $5 \mathrm{~s} 13 \mathrm{~d}$ & ${ }^{3} \mathrm{D}$ & 3 & $148595.76(20)$ & & \\
\hline $5 \mathrm{~s} 13 \mathrm{~d}$ & ${ }^{1} \mathrm{D}$ & 2 & $148630.83(17)$ & & \\
\hline $5 \mathrm{~s} 15 \mathrm{~s}$ & ${ }^{3} \mathrm{~S}$ & 1 & $149050.95(8)$ & 100 & \\
\hline $5 \mathrm{~s} 12 \mathrm{f}$ & ${ }^{3} \mathrm{~F}^{\circ}$ & 3 & $149067.58(24)$ & & \\
\hline $5 \mathrm{~s} 12 \mathrm{f}$ & ${ }^{3} \mathrm{~F}^{\circ}$ & 2 & $149070.69(20)$ & & \\
\hline $5 \mathrm{~s} 15 \mathrm{~s}$ & ${ }^{1} \mathrm{~S}$ & 0 & $149077.3(3)$ & 99 & \\
\hline $5 \mathrm{~s} 12 \mathrm{f}$ & ${ }^{3} \mathrm{~F}^{\circ}$ & 4 & $149078.76(20)$ & & \\
\hline $5 \mathrm{~s} 12 \mathrm{f}$ & ${ }^{1} \mathrm{~F}^{\circ}$ & 3 & $149088.88(20)$ & & \\
\hline $5 \mathrm{~s}_{1 / 2, F=4} 12 \mathrm{~g}$ & & & $149137.36(10)$ & & \\
\hline $5 \mathrm{~s}_{1 / 2, F=5} 12 \mathrm{~g}$ & & & $149140.89(12)$ & & \\
\hline $5 \mathrm{~s}_{1 / 2, F=4} 12 \mathrm{~h}$ & & & $149145.95(16)$ & & \\
\hline $5 \mathrm{~s}_{1 / 2, F=5} 12 \mathrm{~h}$ & & & $149149.57(16)$ & & \\
\hline $5 \mathrm{~s} 14 \mathrm{~d}$ & ${ }^{3} \mathrm{D}$ & 1 & $149165.33(18)$ & & \\
\hline $5 \mathrm{~s} 14 \mathrm{~d}$ & ${ }^{3} \mathrm{D}$ & 2 & $149168.03(25)$ & & \\
\hline $5 \mathrm{~s} 14 \mathrm{~d}$ & ${ }^{3} \mathrm{D}$ & 3 & $149170.15(18)$ & & \\
\hline $5 \mathrm{~s} 14 \mathrm{~d}$ & ${ }^{1} \mathrm{D}$ & 2 & [149196.30(22)] & & RITZPL \\
\hline $5 \mathrm{~s} 16 \mathrm{~s}$ & ${ }^{3} \mathrm{~S}$ & 1 & $149523.97(18)$ & 100 & \\
\hline $5 \mathrm{~s} 16 \mathrm{~s}$ & ${ }^{1} \mathrm{~S}$ & 0 & $149544.20(20)$ & 99 & \\
\hline $5 \mathrm{~s}_{1 / 2, F=4} 13 \mathrm{~g}$ & & & $149590.82(12)$ & & \\
\hline $5 \mathrm{~s}_{1 / 2, F=5} 13 \mathrm{~g}$ & & & $149594.31(12)$ & & \\
\hline $5 \mathrm{~s}_{1 / 2, F=4} 13 \mathrm{~h}$ & & & $149597.77(12)$ & & \\
\hline $5 \mathrm{~s}_{1 / 2, F=5} 13 \mathrm{~h}$ & & & $149601.39(12)$ & & \\
\hline $5 \mathrm{~s} 15 \mathrm{~d}$ & ${ }^{3} \mathrm{D}$ & 1 & {$[149614.22(22)]$} & & RITZPL \\
\hline $5 \mathrm{~s} 15 \mathrm{~d}$ & ${ }^{3} \mathrm{D}$ & 2 & $149614.68(20)$ & & \\
\hline $5 \mathrm{~s} 15 \mathrm{~d}$ & ${ }^{3} \mathrm{D}$ & 3 & $149617.48(18)$ & & \\
\hline $5 \mathrm{~s} 15 \mathrm{~d}$ & ${ }^{1} \mathrm{D}$ & 2 & 149638.1(3) & & \\
\hline $5 \mathrm{~s} 17 \mathrm{~s}$ & ${ }^{3} \mathrm{~S}$ & 1 & 149897.11(20) & 100 & \\
\hline $5 \mathrm{~s} 17 \mathrm{~s}$ & ${ }^{1} \mathrm{~S}$ & 0 & [149913.51(22)] & 99 & RITZPL \\
\hline $5 \mathrm{~s}_{1 / 2, F=4} 14 \mathrm{~g}$ & & & $149950.61(8)$ & & \\
\hline $5 \mathrm{~s}_{1 / 2, F=5} 14 \mathrm{~g}$ & & & $149954.09(7)$ & & \\
\hline $5 \mathrm{~s}_{1 / 2, F=4} 14 \mathrm{~h}$ & & & $149956.21(12)$ & & \\
\hline $5 \mathrm{~s}_{1 / 2, F=5} 14 \mathrm{~h}$ & & & $149959.83(12)$ & & \\
\hline $5 \mathrm{~s} 16 \mathrm{~d}$ & ${ }^{3} \mathrm{D}$ & 1 & $149968.70(25)$ & & \\
\hline $5 \mathrm{~s} 16 \mathrm{~d}$ & ${ }^{3} \mathrm{D}$ & 2 & 149971.5(3) & & \\
\hline $5 \mathrm{~s} 16 \mathrm{~d}$ & ${ }^{3} \mathrm{D}$ & 3 & $149972.30(20)$ & & \\
\hline $5 \mathrm{~s} 16 \mathrm{~d}$ & ${ }^{1} \mathrm{D}$ & 2 & [149988.21(22)] & & RITZPL \\
\hline
\end{tabular}




\begin{tabular}{|c|c|c|c|c|c|}
\hline Configuration & Term & $J$ & $\operatorname{Level}^{\mathrm{a}}\left(\mathrm{cm}^{-1}\right)$ & Leading percentages $^{\mathrm{b}}$ & Comments $^{\mathrm{c}}$ \\
\hline $5 \mathrm{~s} 18 \mathrm{~s}$ & ${ }^{3} \mathrm{~S}$ & 1 & {$[150198.20(22)]$} & 100 & RITZPL \\
\hline $5 \mathrm{~s} 18 \mathrm{~s}$ & ${ }^{1} \mathrm{~S}$ & 0 & {$[150210.90(22)]$} & 99 & RITZPL \\
\hline $5 \mathrm{~s} 17 \mathrm{~d}$ & ${ }^{3} \mathrm{D}$ & 1 & $150258.0(5)$ & & \\
\hline $5 \mathrm{~s} 17 \mathrm{~d}$ & ${ }^{3} \mathrm{D}$ & 2 & $150258.0(5)$ & & \\
\hline $5 \mathrm{~s} 17 \mathrm{~d}$ & ${ }^{3} \mathrm{D}$ & 3 & {$[150258.68(22)]$} & & RITZPL \\
\hline $5 \mathrm{~s} 17 \mathrm{~d}$ & ${ }^{1} \mathrm{D}$ & 2 & $150271.9(3)$ & & \\
\hline $5 \mathrm{~s} 19 \mathrm{~s}$ & ${ }^{3} \mathrm{~S}$ & 1 & {$[150443.52(22)]$} & 100 & RITZPL \\
\hline $5 \mathrm{~s} 19 \mathrm{~s}$ & ${ }^{1} \mathrm{~S}$ & 0 & {$[150453.83(22)]$} & 99 & RITZPL \\
\hline $5 \mathrm{~s} 18 \mathrm{~d}$ & ${ }^{3} \mathrm{D}$ & 1 & $150491.1(3)$ & & \\
\hline $5 \mathrm{~s} 18 \mathrm{~d}$ & ${ }^{3} \mathrm{D}$ & 2 & $150493.5(4)$ & & \\
\hline $5 \mathrm{~s} 18 \mathrm{~d}$ & ${ }^{3} \mathrm{D}$ & 3 & $150493.5(3)$ & & \\
\hline $5 \mathrm{~s} 18 \mathrm{~d}$ & ${ }^{1} \mathrm{D}$ & 2 & {$[150503.81(22)]$} & & RITZPL \\
\hline $5 \mathrm{~s} 19 \mathrm{~d}$ & ${ }^{3} \mathrm{D}$ & 1 & {$[150686.56(22)]$} & & RITZPL \\
\hline $5 \mathrm{~s} 19 \mathrm{~d}$ & ${ }^{3} \mathrm{D}$ & 2 & {$[150687.03(22)]$} & & RITZPL \\
\hline $5 \mathrm{~s} 19 \mathrm{~d}$ & ${ }^{3} \mathrm{D}$ & 3 & $150687.8(3)$ & & \\
\hline In III $\left(5 \mathrm{~s}^{2} \mathrm{~S}_{1 / 2}\right)$ & Limit & & $152200.10(22)$ & & \\
\hline
\end{tabular}

${ }^{a}$ Excitation energies and their uncertainties have been determined from observed wavelengths using the least-squares level optimization code LOPT [23]. Uncertainties in terms of one standard deviation are given in parentheses after the value in the units of the least significant figure of the value. Values enclosed in square brackets correspond to unobserved or poorly measured levels. They were determined semi-empirically using series formulas or a parametric fitting; the method used is specified in the last column.

${ }^{\mathrm{b}}$ The leading percentages have been determined in the present work by means of a parametric least-squares fitting using Cowan's codes [30] (see Sec. 4).

${ }^{\mathrm{c}}$ Methods used in semi-empirical determination of unobserved or poorly measured levels: RITZPL and POLAR - Ritz-type quantumdefect and polarization formulas fitted with Sansonetti's computer codes [29]; LSF - Parametric least-squares fitting with Cowan's codes [30].

shift (IS) for $\mathrm{In}^{+}$was made by Wang et al. [27]. These authors have measured the IS between the energies of the $5 \mathrm{~s} 5 \mathrm{p}^{3} \mathrm{P}^{\circ}{ }_{1}$ level in ${ }^{113} \mathrm{In}^{+}$and ${ }^{115} \mathrm{In}^{+}$to be $0.02321(6) \mathrm{cm}^{-1}$. Since the abundance of ${ }^{113} \mathrm{In}$ in natural samples is only $4.3 \%$ [6], the value of the $5 \mathrm{~s} 5 \mathrm{p}{ }^{3} \mathrm{P}^{\circ}$ level for the natural mixture of indium isotopes is lower than that of ${ }^{115} \mathrm{In}^{+}$by $0.0010 \mathrm{~cm}^{-1}$. Most of the levels given in Table 2 are ultimately based on $5 \mathrm{~s} 5 \mathrm{p}^{3} \mathrm{P}^{\circ}$. Therefore, they are systematically too high by this amount. Since the uncertainties of the level values are much greater, this shift has been neglected.

\section{Theoretical Interpretation of the Energy Levels}

To find eigenvector compositions of the levels and calculate transition probabilities, I used a parametric fitting with Cowan's computer codes [30]. The following configurations were included in the calculations: $5 \mathrm{~s}^{2}, 5 \mathrm{~s} n \mathrm{~s}(n=6-19), 5 \mathrm{~s} n \mathrm{~d}(n=5-10), 5 \mathrm{~s} n \mathrm{~g}(n=5-10)$, and $5 \mathrm{p}^{2}$ for even parity, and $5 \mathrm{~s} n \mathrm{p}(n=5-10)$, $5 \mathrm{~s} n \mathrm{f}(n=4-10)$, and $5 \mathrm{~s} n \mathrm{~h}(n=6-10)$ for odd parity. In the even parity set, 79 known levels were fitted with 35 free parameters with an average deviation of $16 \mathrm{~cm}^{-1}$. In the odd parity set, 60 known levels were fitted with 25 free parameters with an average deviation of $13 \mathrm{~cm}^{-1}$. Percentage compositions of the levels included in Table 2 are from these calculations. The fitted parameters were used to calculate the transition probabilities.

The percentage compositions of the levels given in Table 2 result from the parametric fitting described above. In this table, the energies of the $5 \mathrm{~s} n \mathrm{p}^{3} \mathrm{P}_{0}^{\circ}$ levels with $n=8-10$, which were not observed experimentally, are from the same parametric fitting. The value for $5 \mathrm{~s} 8 \mathrm{p}^{3} \mathrm{P}_{0}^{\circ}, 135823(10) \mathrm{cm}^{-1}$, is in fair agreement with the result of the parametric fitting by Biémont and Zeippen [35], $135833 \mathrm{~cm}^{-1}$. 


\section{Interpretation of the $5 \mathrm{sng}$ and $5 \mathrm{snh}$ Series}

Paschen and Campbell [2] found that the $5 \mathrm{~s} n \mathrm{~g}$ and $5 \mathrm{~s} n \mathrm{~h}$ configurations are split into pairs of closely located level groups, which they designated as $n-\mathrm{G}, n+\mathrm{G}, n-\mathrm{H}$, and $n+\mathrm{H}$. The $5 \mathrm{~s} n \mathrm{~g}$ series was observed for $n=5-14$, and the $5 \mathrm{~s} n$ h series for $n=8-14$. The separations within the pairs (e.g., between $n-\mathrm{G}$ and $n+\mathrm{G}$ ) are almost constant along the series and are equal to $3.56(3) \mathrm{cm}^{-1}$ for the $5 \mathrm{~s} n \mathrm{~g}(n=7-14)$ series and $3.58(8) \mathrm{cm}^{-1}$ for the $5 \mathrm{~s} n \mathrm{~h}(n=8-14)$. The separations within the $5 \mathrm{~s} 5 \mathrm{~g}$ and $5 \mathrm{~s} 6 \mathrm{~g}$ level pairs are slightly greater, $3.91(8) \mathrm{cm}^{-1}$ and $3.71(10) \mathrm{cm}^{-1}$, respectively. The almost constant separations for $n \geq 7$ imply that their probable cause is the hyperfine splitting of the In III $5 \mathrm{~s}$ core. Indeed, the predicted hfs interval in the

${ }^{115}$ In III $5 \mathrm{~s}$ ground state is $3.56 \mathrm{~cm}^{-1}$ [31]. There are no measurements of this interval in In III. However, it was measured in the isoelectronic spectra of ${ }^{107} \mathrm{Ag} \mathrm{I}$ [32] and ${ }^{111} \mathrm{Cd}$ II [33]. These measurements agree with the calculations of Beck and Datta [31] within $3 \%$, which suggests that their result for ${ }^{115}$ In III quoted above is similarly accurate. The close agreement between the theoretical hfs interval of ${ }^{115} \mathrm{In}$ III $5 \mathrm{~s}$ and the observed separations in the In II $5 \mathrm{~s} n \mathrm{~g}$ and $5 \mathrm{~s} n \mathrm{~h}$ series suggests that these observed separations represent the first measurement of the hfs interval in ${ }^{115} \mathrm{In}$ III $5 \mathrm{~s}$. The weighted mean of this interval, averaged over the high members of both the $5 \mathrm{~s} n \mathrm{~g}$ and $5 \mathrm{~s} n \mathrm{~h}$ series $(n \geq 7)$ is $3.56(3) \mathrm{cm}^{-1}$.

Thus, these two series represent the first observation of an unusual coupling between the fine-structure and hfs interactions in an atomic system where the $\mathrm{hfs}$ interaction dominates over the fine-structure effects such as the spin-orbit splitting and electrostatic exchange. According to my Cowan code calculations (see the previous section), the average fine-structure splittings caused by the spin-orbit splitting and electrostatic exchange are the largest for the $5 \mathrm{~s} 5 \mathrm{~g}$ configuration and amount to about $2 \mathrm{~cm}^{-1}$. They rapidly decrease with increasing $n$ along the $5 \mathrm{~s} n$ g series and are smaller than $1 \mathrm{~cm}^{-1}$ for $n \geq 9$. For all members of the $5 \mathrm{~s} n \mathrm{~h}$ series, the fine-structure splittings are smaller than $0.0002 \mathrm{~cm}^{-1}$. These splittings are indeed much smaller than the hfs interval in ${ }^{115}$ In III $5 \mathrm{~s}$, which may explain the observed constant intervals within the pairs along the series, as well as the increased intervals in $5 \mathrm{~s} 5 \mathrm{~g}$ and $5 \mathrm{~s} 6 \mathrm{~g}$. A similar behavior of the level intervals along the $1 \mathrm{~s} n \mathrm{f}, 1 \mathrm{~s} n \mathrm{~g}, 1 \mathrm{~s} n \mathrm{~h}, 1 \mathrm{~s} n \mathrm{i}$, and $1 \mathrm{~s} n \mathrm{k}$ series was predicted by Morton et al. [34] for ${ }^{3} \mathrm{He} \mathrm{I}$, where the hfs interval in the ${ }^{3} \mathrm{He}$ II $1 \mathrm{~s}$ core, $-8666 \mathrm{MHz}$, is much greater (in absolute value) than the fine-structure splittings. The latter are smaller than $400 \mathrm{Mhz}$ for all members of these series.

Because of this unusual coupling, no $L S$ term designations can be assigned to the $5 \mathrm{~s} n \mathrm{~g}$ and $5 \mathrm{~s} n \mathrm{~h}$ series. Instead, I designate Paschen and Campbell's $n-\mathrm{G}, n+\mathrm{G}, n-\mathrm{H}$, and $n+\mathrm{H}$ levels as $5 \mathrm{~s}_{1 / 2, F=4} n \mathrm{~g}, 5 \mathrm{~s}_{1 / 2, F=5} n \mathrm{~g}$, $5 \mathrm{~s}_{1 / 2, F=4} n \mathrm{~h}$, and $5 \mathrm{~s}_{1 / 2, F=5} n \mathrm{~h}$, respectively. Each of these levels is comprised of multiple sublevels arising from coupling of the angular momentum $F$ of the core with the angular momentum $J_{2}$ of the outer electron. For the lower members of the $5 \mathrm{~s} n \mathrm{~g}$ series, the intervals between the sublevels are comparable to the interval between the $F=4$ and $F=5$ hyperfine components of the core, while for the higher members of this series, as well as for the entire $5 \mathrm{~s} n \mathrm{~h}$ series, they are much smaller.

\section{Relative Intensities of Observed Lines}

As noted in the Introduction, relative intensities of lines observed with different light sources and with different registration equipment are vastly different. In order to give a consistent set of relative intensities, they must be converted to the same scale. To account for the different excitation conditions in various light sources, the observed line intensities can be approximated by local thermodynamic equilibrium (LTE) with certain excitation temperature pertinent to each light source, and then scaled to the same excitation temperature. In reality, the LTE approximation describes the observed intensities only qualitatively, with deviations in both directions up to an order of magnitude. However, this method results in much better qualitative agreement between relative intensities observed by different authors. Besides the different effective temperatures in the light sources, the observed intensities are strongly affected by different behaviors of spectral response functions of the registration equipment at different wavelengths. These variations can also be accounted for and removed. These procedures are described below. The general method relies on radiative transition rates $A_{k i}$ calculated with Cowan's codes (see Sec. 4), and on the LTE relation between these transition rates and the observed intensities $I_{\mathrm{obs}}$ : 


$$
I_{\mathrm{obs}} \propto\left(g_{k} A_{k i} / \lambda\right) \exp \left(-E_{k} / k T_{\text {eff }}\right),
$$

where $g_{\mathrm{k}}$ and $E_{k}$ are the statistical weight and energy of the upper level, $\lambda$ is the central wavelength of the line, $k$ is the Boltzmann constant, and $T_{\text {eff }}$ is the effective temperature.

\subsection{Line Intensities in the Spectra Observed by Karlsson and Litzén [11]}

As noted in Sec. 2.1, the intensities given by Karlsson and Litzén [11] for their FTS measurements are roughly the signal-to-noise ratios. The effective temperature can be easily found from the Boltzmann plot, i.e., from the linear slope of $I_{\mathrm{obs}} \lambda /\left(g_{k} A_{k i}\right)$ versus the energy of the upper level $E_{k}$. Intensities reported by Karlsson and Litzén [11] are well described by effective temperature of $T_{\text {eff }} \approx 1.1 \mathrm{eV}$. No wavelength dependence of the registration sensitivity was found for their observations.

\subsection{Line Intensities in the Spectra Observed by Paschen and Campbell [2]}

The analysis of line intensities reported by Paschen and Campbell [2] is complicated due to the large number of different registration methods used. The analysis is simplest for the observations made with the single-prism spectrograph. These observations span the wavelength region between $2100 \AA$ and $6630 \AA$, but most of them are below $2700 \AA$. The intensities reported by Paschen and Campbell are visual estimates of blackening of photographic plates; hence, they are non-linear in response to exposure. This non-linearity should be removed together with the wavelength dependence of the sensitivity. This was done in an iterative procedure, which resulted in an effective temperature $T_{\text {eff }} \approx 3.4 \mathrm{eV}$. The spectral variation of the sensitivity can be visualized by plotting the logarithm of the ratio of calculated and observed intensities $I_{\text {obs }} / I_{\text {calc }}$, where $I_{\text {calc }}$ is set to equal the right side of Eq. (4), against wavelength. This plot for the single-prism observations is presented in Fig. 1.

As seen from this figure, the sensitivity of the single-prism observations exponentially drops by three orders of magnitude when the wavelength decreases from $2700 \AA$ to $2100 \AA$.

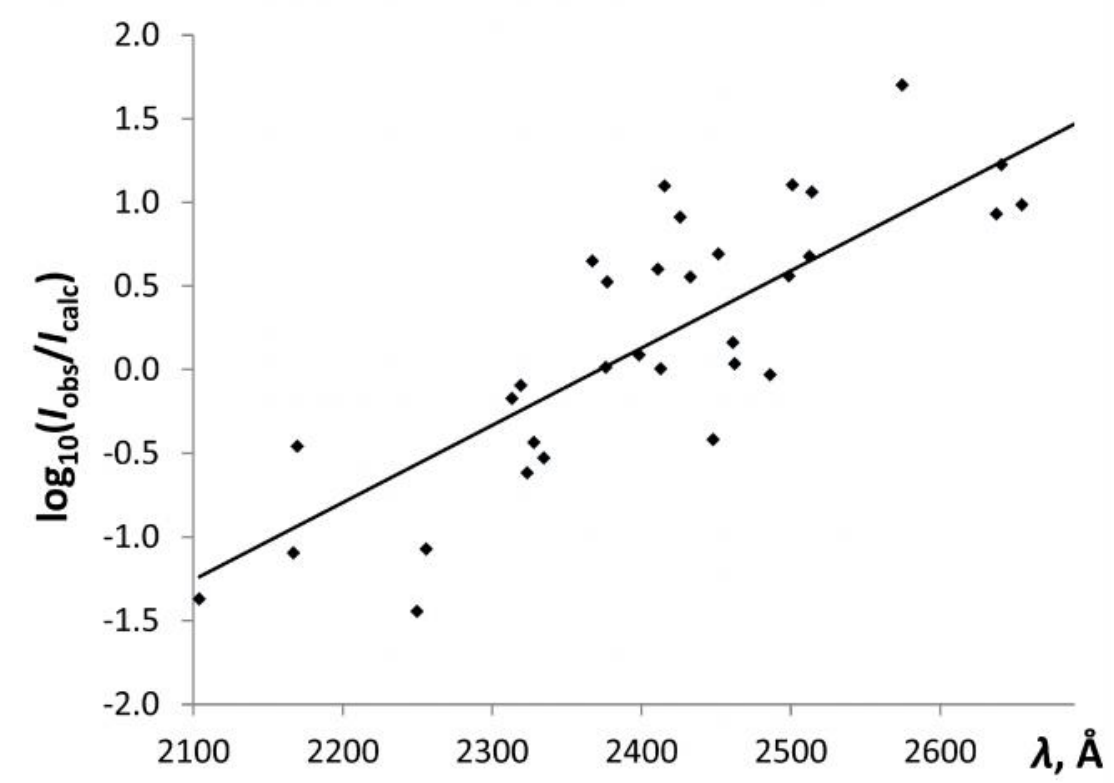

Fig. 1. A logarithmic plot of the spectral sensitivity of single-prism observations of Paschen and Campbell [2] against wavelength. The solid line is a linear least-squares fit to the data points. 
Similar plots were built for observations made with the three-prism spectrograph and with the grating spectra in various orders of diffraction. As a sample, the plot of the spectral sensitivity of the grating observations in the first order of diffraction is given in Fig. 2.

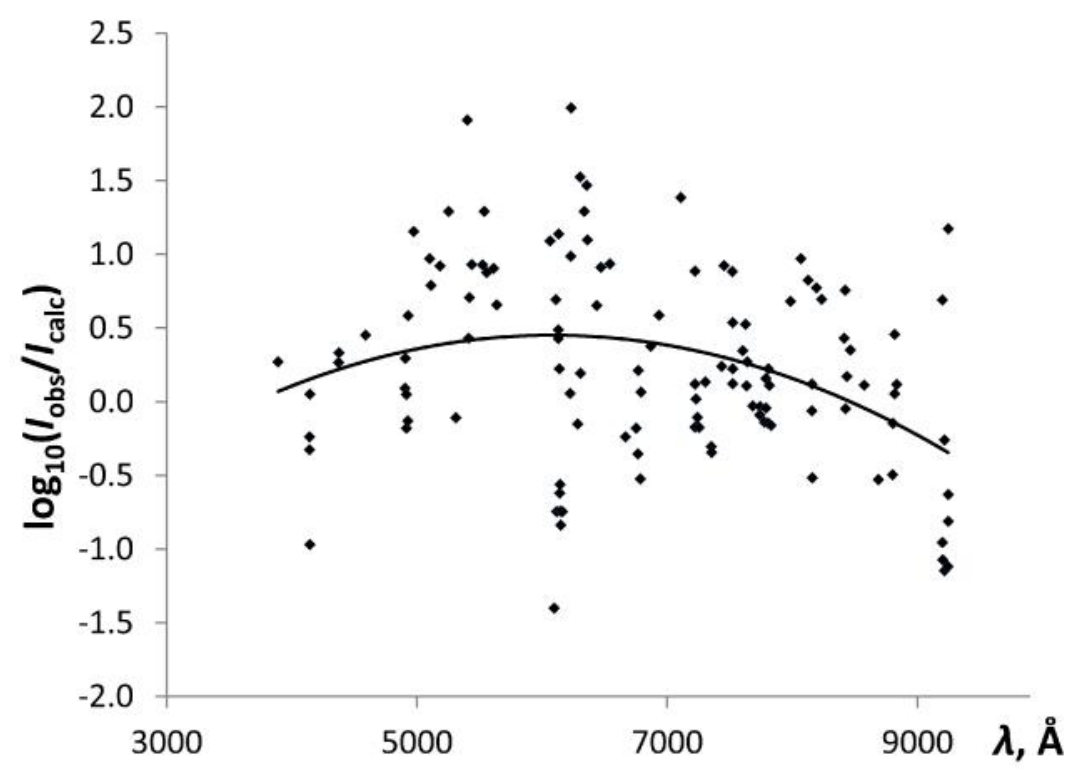

Fig. 2. A logarithmic plot of the spectral sensitivity of grating observations of Paschen and Campbell [2] in the first order of diffraction against wavelength. The solid line is a quadratic least-squares fit to the data points.

To verify that the non-linearity was correctly removed from the intensity values, the logarithm of the calculated intensities is plotted against the corrected observed intensities in Fig. 3. In this plot, the wavelength dependence was removed from all observations.

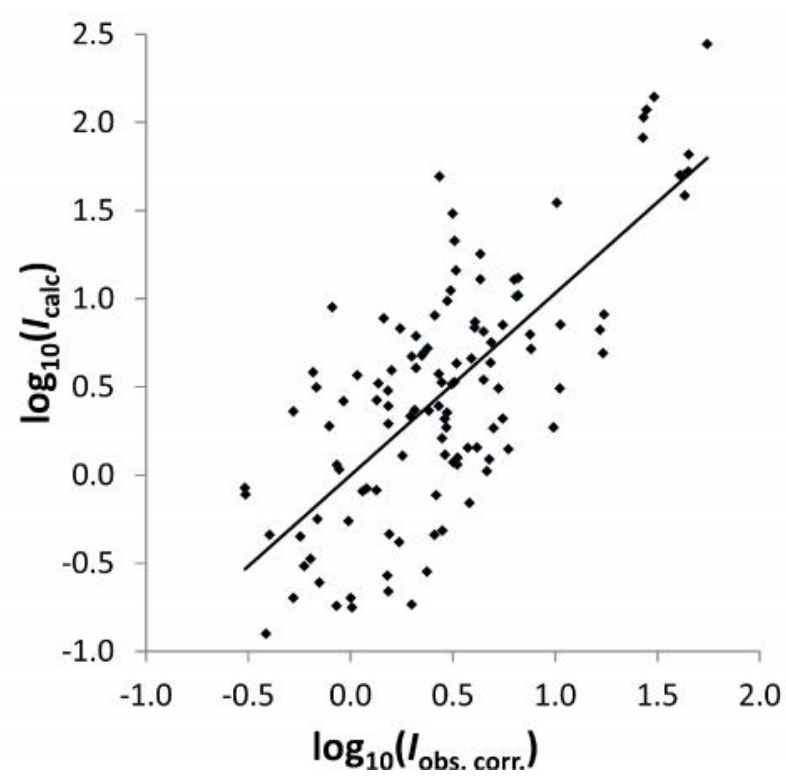

Fig. 3. Verification of linearity of the corrected intensity scale for the observations of Paschen and Campbell [2]. The solid curve is a linear fit to the data points. Its slope is close to one, and it nearly crosses the $(0,0)$ point. 
Removal of the wavelength dependence of sensitivity and non-linearity of the intensity scale has a dramatic effect on the values of relative intensities. For example, the ratio of relative intensities of the allowed $5 \mathrm{~s}^{2}{ }^{1} \mathrm{~S}_{0}-5 \mathrm{~s} 5 \mathrm{p}^{3} \mathrm{P}^{\circ}{ }_{1}$ transition at $2306 \AA$ and the highly forbidden hyperfine-induced $5 \mathrm{~s}^{2}{ }^{1} \mathrm{~S}_{0}-5 \mathrm{~s} 5 \mathrm{p}{ }^{3} \mathrm{P}_{0}^{\circ}$ transition at $2365 \AA$, as given by Paschen and Campbell [2], is 3.3, while the ratio of the corrected intensities is 75 .

\subsection{Line Intensities in the Spectra Observed by Bhatia [5]}

Analysis of intensities observed by Bhatia [5] presented the greatest difficulties for several reasons. First, as noted in Sec. 2.4, the great majority of the lines observed in this work belong to higher ionization stages. Out of the total 4000 lines, only about 300 could be ascribed to In II. Thus, blending with lines of other ionization stages can be expected to distort intensities of many In II lines. Second, a significant number of the lines were overexposed. Due to a strong non-linearity of the response of photographic plates at high exposures, intensities of strong lines are greatly underestimated. Therefore, when analyzing the intensities from Bhatia [5], overexposed lines and lines suspected to be perturbed by other ionization stages were excluded from consideration.

The analysis was made using an approach similar to the one described above. Namely, the intensities of the lines in different spectral regions were treated separately, assuming that the response of the registration equipment has a different dependence on wavelength in those regions.

The effective excitation temperature describing the line intensities observed by Bhatia was found to be about $8 \mathrm{eV}$. This is much higher than the temperature found for all other observations, which is consistent with the fact that most of the lines observed in Bhatia's experiment belong to higher ionization stages.

\subsection{Line Intensities in the Spectra Observed by Lang and Sawyer [3]}

As noted in Sec. 2.5, Lang and Sawyer [3] used three different spectrographs, a $1 \mathrm{~m}$ vacuum grating spectrograph and two prism spectrographs, to photograph the spectrum between $500 \AA$ and $8000 \AA$. They did not specify the spectral regions in which each of these spectrographs was used. However, by making Boltzmann plots similar to those described in the previous section it was possible to determine the effective temperature of their light source, $T_{\text {eff }} \approx 2.5 \mathrm{eV}$, and divide the entire spectral range of their observations into three separate regions with distinctly different variation of spectral sensitivity. This division is illustrated in Fig. 4, which depicts the dependence of the logarithm of the ratio of observed and calculated intensities.

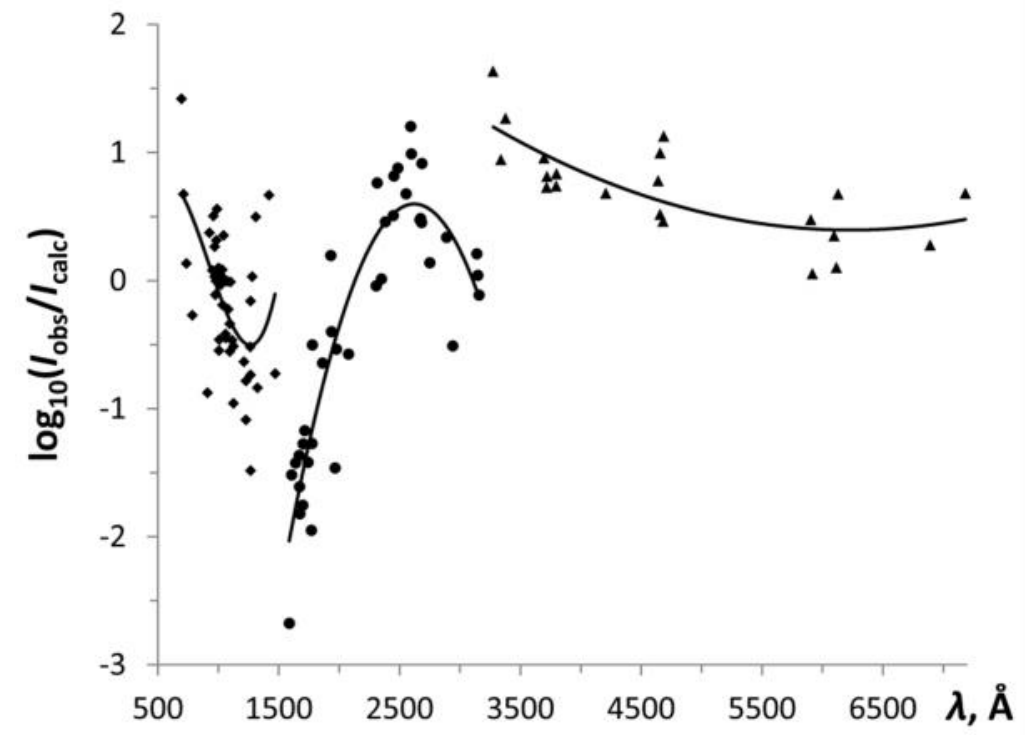

Fig. 4. A logarithmic plot of the spectral sensitivity of experimental set-up of Lang and Sawyer [3] in three wavelength regions, $500 \AA$ to $1500 \AA, 1500 \AA$ to $3200 \AA$, and $3200 \AA$ to $7000 \AA$. The solid lines are polynomial least-squares fits to the data points (cubic for the first two regions, and quadratic for the last one). 


\subsection{Line Intensities in the Spectra Observed by Wagatsuma [15]}

By using a similar method as described above, I determined the effective excitation temperatures for the three sets of measurements presented by Wagatsuma. For the spectrum obtained with neon buffer gas, the effective temperature was $T_{\text {eff }} \approx 2.1 \mathrm{eV}$; for the Ar-He mixture, $T_{\text {eff }} \approx 2.0 \mathrm{eV}$; and for pure argon, $T_{\text {eff }} \approx$ $1.2 \mathrm{eV}$. Since the greatest number of lines was observed with neon buffer gas, I reduced all intensity measurements to $T_{\text {eff }}=2.1 \mathrm{eV}$ and averaged all intensities observed in more than one set-up.

\subsection{Reduction of Intensities to a Uniform Scale}

After the intensities reported by different observers were corrected for variations of the response functions, as described above, they were reduced to a uniform scale by scaling them to the same effective excitation temperature $T^{*}$. This temperature was chosen to be about $3 \mathrm{eV}$, since the most extensive line list given by Paschen and Campbell is described by a temperature close to that value (see Sec. 6.2). The scaling was made by multiplying the intensity values by a factor $f_{\mathrm{sc}} \exp \left[-E_{k}\left(1 / k T^{*}-1 / k T_{\text {eff }}\right)\right]$, where the scaling coefficient $f_{\mathrm{sc}}$ was determined by adjusting the scale so that the mean ratio of intensities to those of Paschen and Campbell is equal to one. After that, intensities from multiple observations were averaged. The resulting values are reported in Table 1 . About $70 \%$ of lines have only one intensity measurement. Most of them are from Paschen and Campbell [2], while about 90 are from Bhatia [5], 22 from Lang and Sawyer [3], two from Wagatsuma [15], and one from Karlsson and Litzén [11]. For 181 lines, intensities have been determined by averaging two or more measurements. For 17 lines, relative intensities resulting from measurements of different authors differ by more than an order of magnitude. These highly volatile values, as well as five other intensities that may have been affected by blending with other lines, have been marked as unreliable in Table 1.

\section{Transition Probabilities}

Calculated or measured transition probabilities and radiative lifetimes of In II are given in more than 30 published articles. The complete list of these articles can be obtained from the NIST Atomic Transition Probability Bibliographic Database [36]. Here I will discuss only the articles that provide the most dependable data.

Becker et al. [37] measured the radiative lifetime of the metastable $5 \mathrm{~s} 5 \mathrm{p}^{3} \mathrm{P}^{\circ}{ }_{0}$ level of a single lasercooled ${ }^{115} \mathrm{In}^{+}$ion in a radiofrequency trap. The value they obtained, $0.195(8) \mathrm{s}$, corresponds to the probability of 5.13(21) $\mathrm{s}^{-1}$ for the hyperfine-induced transition to the ground state. These authors also measured the Landé factor of the $5 \mathrm{~s} 5 \mathrm{p}{ }^{3} \mathrm{P}_{0}^{\circ}$ level, $-0.000987(5)$. As well as the non-zero decay probability, the non-zero value of the Landé factor is caused by perturbations induced by the hyperfine interactions.

Ansbacher et al. [38] measured radiative lifetimes for 13 levels of In II using the beam-foil method with an account for cascade corrections. These measurements provided a base for the most accurate transition probability values of transitions originating from those levels. For two levels, $5 \mathrm{~s} 5 \mathrm{~d}^{3} \mathrm{D}_{3}$ and $5 \mathrm{~s} 4 \mathrm{f}^{3} \mathrm{~F}^{\circ}{ }_{4}$, there is only one branch of radiative decay to the $5 \mathrm{~s} 5 \mathrm{p}^{3} \mathrm{P}_{2}^{\circ}$ and $5 \mathrm{~s} 5 \mathrm{~d}^{3} \mathrm{D}_{3}$ levels, respectively, so the measured lifetimes directly yield the transition probabilities. For each of the other eight levels $\left(5 \mathrm{~s} 6 \mathrm{~s}{ }^{1} \mathrm{~S}_{0}, 5 \mathrm{~s} 6 \mathrm{~s}^{3} \mathrm{~S}_{1}\right.$, $5 \mathrm{~s} 5 \mathrm{~d}^{1} \mathrm{D}_{2}, 5 \mathrm{~s} 5 \mathrm{~d}^{3} \mathrm{D}_{1}, 5 \mathrm{~s} 5 \mathrm{~d}^{3} \mathrm{D}_{2}, 5 \mathrm{p}^{2}{ }^{3} \mathrm{P}_{2}, 5 \mathrm{p}^{2}{ }^{1} \mathrm{D}_{2}$, and $5 \mathrm{p}^{2}{ }^{1} \mathrm{~S}_{0}$ ) radiative decay is dominated by one transition. For these levels, the transition probability of the dominant decay branch could be accurately determined by subtracting from the total decay rate (i.e., inverse of the measured lifetime) the sum of the contributions of the other weak decay branches, which were accurately calculated by other authors. Uncertainties of thus determined transition probabilities vary between $3 \%$ and $40 \%$.

Curtis et al. [39] made an isoelectronic comparison of measured and calculated radiative lifetimes of the $5 \mathrm{~s} 5 \mathrm{p}^{1} \mathrm{P}_{1}^{\circ}$ and $5 \mathrm{~s} 5 \mathrm{p}^{3} \mathrm{P}^{\circ}{ }_{1}$ levels for Cd-like spectra of elements between $\mathrm{Cd}$ and Ho. Their semiempirically corrected values for In II are probably accurate to within a few percent. They agree well with the best available measurements [38,40].

Martinez et al. [41] measured the branching fractions of transitions from the four $5 \mathrm{~s} 4 \mathrm{f}$ levels using an optically thin laser-produced plasma. From these data, they derived transition probabilities ( $A$-values) for 
10 transitions by normalizing them to the lifetimes measured by Ansbacher et al. [38] (for $5 \mathrm{~s}^{4} \mathrm{f}^{1} \mathrm{~F}_{3}^{\circ}$ and $5 \mathrm{~s} 4 \mathrm{f}^{3} \mathrm{~F}_{3,4}^{\circ}$ ) and by Blagoev et al. [42] (for $5 \mathrm{~s} 4 \mathrm{f}^{3} \mathrm{~F}^{\circ}{ }_{2}$ ). For these $A$-values, they gave uncertainty values ranging from $10 \%$ to $35 \%$. I have adopted their $A$-values that were normalized to the lifetimes measured by Ansbacher et al. Comparison of different measurements and accurate calculations shows that the lifetimes measured by Blagoev et al. [42] cannot be trusted. In most cases, they are too low by up to a factor of 2.

Jönsson and Andersson [43] made extensive relativistic multiconfiguration Dirac-Hartree-Fock calculations of oscillator strengths for electric-dipole transitions in In II. They gave weighted oscillator strengths $(g f)$ calculated in both Babushkin and Coulomb gauges, and provided for each transition their calculated wave number. Comparison of the values in the two gauges provides a rough estimate of average uncertainty of their $g f$ values, which is about $15 \%$. The given wave numbers allowed me to adjust their $g f$ values by re-scaling them to the accurate Ritz wave numbers and convert to the $A$-values. Comparison of the resulting $A$-values with the accurate data discussed above shows that there is a strong dependence of the accuracy of the calculated $A$-values on the line strength $S$. It turned out that the $A$-values for $S>3$, as calculated by Jönsson and Andersson, are accurate to within $8 \%$, while for weaker transitions the accuracy is much worse, $33 \%$ on average. The agreement between the $g f$ values in Babushkin and Coulomb gauges was used as an additional indicator of accuracy. I have decreased the assumed accuracy for transitions for which the $g f$ values in the two gauges differ by more than $15 \%$.

Biémont and Zeippen [35] calculated the oscillator strengths for a large number of electric dipole transitions in In II using Cowan's Hartree-Fock Relativistic method [30] modified to account for corepolarization effects by including a semi-empirically determined model potential. The radial parameters of the wave functions were determined in a least-squares fitting of the experimentally known energy levels. I have compared their results with an extended base of accurately determined $A$-values that included the accurate results from Jönsson and Andersson [43] in addition to other results discussed above. For strong transitions with $S>1.25$, calculations of Biémont and Zeippen agree with other accurate results with an average deviation of $18 \%$. For transitions with $S$ between 0.2 and 1.25 , the average deviation is about $30 \%$. For weaker transitions, the agreement is much worse, often only to an order of magnitude.

Lavín and Martin [44] employed the relativistic quantum defect orbital (RQDO) approach with an account for core polarization to calculate the oscillator strengths of the $5 \mathrm{~s} 5 \mathrm{p}{ }^{3} \mathrm{P}-5 \mathrm{~s} n \mathrm{~d}^{3} \mathrm{D}(n=5-9)$ and $5 \mathrm{~s} 5 \mathrm{p}{ }^{3} \mathrm{P}-5 \mathrm{~s} n \mathrm{~s}{ }^{3} \mathrm{~S}(n=6-10)$ transitions in In II and similar transitions in a few neighboring isoelectronic spectra. Where the scope of their calculations intersects the scope of the other works considered above $[38,35,43]$, their results agree to about $25 \%$ with those references with no discernible correlation with the line strength.

The works discussed above provided an extensive database for comparison with my semi-empirical Cowan-code calculations. It is well known that Cowan-code calculations of transition rates suffer from cancellation effects. To check whether these effects are significant, the Cowan code provides the value of the cancellation factor in the output for each transition. The cancellation factor is computed as follows. The line strength is computed as an expansion over contributions from each basis state. The sum of positive contributions $S^{+}$and the sum of negative contributions $S$ are computed separately, and the cancellation factor is calculated as $\mathrm{CF}=\left(S^{+}+S^{-}\right) /\left(S^{+}+\left|S^{-}\right|\right)$. Since each of $S^{+}$and $S^{-}$are calculated with a finite accuracy, the values of CF close to zero indicate a significant loss of accuracy in the final line strength. Of course, this is only a qualitative criterion. Different researchers adopt different threshold values of CF below which they consider the results to be adversely affected by cancellations. In the case of the In II calculations, my comparisons show that there is no correlation between the accuracy of line-strength calculations and $\mathrm{CF}$ for $|\mathrm{CF}|$ greater than 0.1 . However, the values of $|\mathrm{CF}|<0.1$ generally indicate that the resulting line strength can be in error by an order of magnitude or more.

Considering only transitions unaffected by cancellations, I found that, similar to several other calculation methods discussed above, the accuracy of Cowan-code calculations strongly depends on the line strength. For strongest transitions with $S>80$, the results of Cowan's code agree with other, more accurate calculations or measurements within $9 \%$ on average. For strong transitions with $S$ between 2.5 and 80 , the accuracy is about $23 \%$ on average. For weaker transitions with $S<2.5$, the average accuracy is about $30 \%$. I have discarded most of the $A$-values from the Cowan code calculations that were adversely affected by cancellation effects (i.e., those that have $\mathrm{CF}<0.1$ ). Only a few of such unreliable $A$-values are 
included in Table 1 for transitions that have observed intensities roughly agreeing with those calculated using these $A$-values in the assumption of LTE.

Comparisons of radiative lifetimes measured by different authors with calculations were given in many papers; see, for example, Biémont and Zeippen [35]. These comparisons show that the various measurements strongly disagree with each other for many energy levels. It can be seen that the lifetimes measured by Ansbacher et al. [38] with an account for multi-exponential decay are in much better agreement with theory than the other measurements. Some of the lifetimes measured by other authors may be significantly affected by cascades from higher levels. For example, there is a large disagreement between theory and measurements for the $5 \mathrm{~s} 5 \mathrm{f}^{3} \mathrm{~F}^{\circ}{ }_{4}$ level. For this level, Blagoev et al. [42] measured a lifetime of 19(1) ns, in agreement with an earlier beam-foil measurement by Andersen and Sørensen [45], 21(2) ns. Different calculations yield consistently lower values, $11.8 \mathrm{~ns}$ [43], $10.0 \mathrm{~ns}$ [35], or $9.5 \mathrm{~ns}$ (this work). As follows from my calculations, the measured lifetime probably corresponds to the cascade from $5 \mathrm{~s} 6 \mathrm{~g}$, for which my calculations yield a lifetime of $21.3 \mathrm{~ns}$. Similarly, for the $5 \mathrm{~s} 5 \mathrm{f}^{3} \mathrm{~F}_{3}^{\circ}$ level, Andersen and Sørensen [45] reported a lifetime of 21(2) ns, while three different calculations yield $11.7 \mathrm{~ns}$ [43], $9.9 \mathrm{~ns}$ [35], and $9.4 \mathrm{~ns}$ (this work). Similar to the $5 \mathrm{~s} \mathrm{f}^{3} \mathrm{~F}^{\circ}{ }_{4}$ level, the measured $5 \mathrm{~s} 5 \mathrm{f}^{3} \mathrm{~F}^{\circ}{ }_{3}$ lifetime appears to correspond to the cascade from $5 \mathrm{~s} 6 \mathrm{~g}$.

The critically evaluated transition probabilities with assessed uncertainties are compiled in Table 1 . The uncertainties are denoted by a letter code explained in Table 3.

Table 3. Transition probability uncertainty code

\begin{tabular}{lll}
\hline \hline Letter & $\begin{array}{l}\text { Uncertainty } \\
\text { in } A \text {-value }\end{array}$ & $\begin{array}{l}\text { Uncertainty } \\
\text { in } \log (g f)\end{array}$ \\
\hline $\mathrm{A}$ & $\leq 3 \%$ & $\leq 0.013$ \\
$\mathrm{~B}+$ & $\leq 7 \%$ & $\leq 0.03$ \\
$\mathrm{~B}$ & $\leq 10 \%$ & $\leq 0.04$ \\
$\mathrm{C}+$ & $\leq 18 \%$ & $\leq 0.08$ \\
$\mathrm{C}$ & $\leq 25 \%$ & $\leq 0.11$ \\
$\mathrm{D}+$ & $\leq 40 \%$ & $\leq 0.18$ \\
$\mathrm{D}$ & $\leq 50 \%$ & $\leq 0.24$ \\
$\mathrm{E}$ & $>50 \%$ & $>0.24$ \\
\hline
\end{tabular}

It should be noted that the transition probability values resulting from theoretical calculations do not follow a normal statistical distribution. For example, for normal statistics the uncertainty of $\pm 10 \%$ (at the one standard deviation level) would imply that the true value should be within $20 \%$ of the measured or calculated one with a probability of $95 \%$, and a deviation of $30 \%$ has an extremely small probability. However, in quantum mechanics calculations it is often observed that the bulk of results for $A$-values lie within certain limits from true values, but there are some $A$-values (usually for weak transitions) that deviate grossly (by an order of magnitude or more) from the true values. This is the reason for using the above letter codes rather than the usual numerical specification of uncertainties. The numerical uncertainty values given in Table 3 should be used with caution, since there may be a significant number of outliers one would not expect to have in normal statistics. The logarithms of the $A$-values, as well as the values of $\log (g f)$, have statistical distributions much closer to normal.

\section{Conclusions}

In the present study, a comprehensive list of the best measured wavelengths of 650 observed lines has been compiled. Uncertainties of all available wavelength measurements in the In II spectrum have been analyzed, and the existing inconsistencies have been resolved. From this line list with assigned uncertainties, a set of 173 optimized energy levels that fits all observed wavelengths has been derived. An additional 21 levels have been determined semi-empirically from series formulas and from parametric 
fitting. Percentage compositions of the levels and radiative transition rates have been calculated in the parametric fitting procedure. An improved value of the ionization limit of In II has been determined by fitting quantum-defect and polarization formulas for several series of levels. The line intensities observed by different authors have been analyzed and converted to a uniform scale. A set of 528 recommended values of radiative transition rates has been critically compiled, and uncertainties of these rates have been estimated. Of these transition rates, 353 are associated with observed lines. From the observed separations of levels in the $5 \mathrm{~s} n \mathrm{~g}$ and $5 \mathrm{~s} n \mathrm{~h}$ series of In II, the hfs splitting of the ground state of ${ }^{115} \mathrm{In}$ III has been found to be $3.56(3) \mathrm{cm}^{-1}$.

\section{Acknowledgments}

This work was partially supported by the Office of Fusion Energy Sciences of the US Department of Energy and by the National Aeronautics and Space Administration. I am grateful to W. C. Martin for fruitful discussions and careful reading of the manuscript.

\section{References}

[1] A. Kramida, NIST Atomic Energy Levels and Spectra Bibliographic Database (2012), available at: http://physics.nist.gov/cgibin/ASBib1/Elevbib/make query.cgi?spectra=In\%20II\&order $=1$.

[2] F. Paschen and J. S. Campbell, Ann. Phys. (Leipzig) 423, 29 (1938). http://dx.doi.org/10.1002/andp.19384230103

[3] R. J. Lang and R. A. Sawyer, Z. Phys. 71, 453 (1931). http://dx.doi.org/10.1007/BF01340078

[4] C. E. Moore, Atomic Energy Levels as Derived from the Analysis of Optical Spectra - Molybdenum through Lanthanum and Hafnium through Actinium, Nat. Bur. Stand. Circular 467, Vol. III (1958), 245 pp; reprinted in Nat. Stand. Ref. Data Ser. 35, Vol. III (1971).

[5] K. S. Bhatia, Analysis of Doubly and Triply Ionised Indium, Ph.D. Thesis, University of British Columbia, Canada, 182 pp. (1969). https://circle.ubc.ca/handle/2429/35526

[6] J. E. Sansonetti and W. C. Martin, "Handbook of Basic Atomic Spectroscopic Data," J. Phys. Chem. Ref. Data 34, 1559 (2005), available at: http://physics.nist.gov/PhysRefData/Handbook/index.html. http://dx.doi.org/10.1063/1.1800011

[7] H. Dehmelt, Mono-Ion Oscillator as Potential Ultimate Laser Frequency Standard, in Proceedings of the Thirty Fifth Annual Frequency Control Symposium (Electronic Industries Association, Washington, DC, USA, 1981), pp. 596-601; see also H. G. Dehmelt, IEEE Trans. Instrum. Meas. 31, 83 (1982). http://dx.doi.org/10.1109/TIM.1982.6312526

[8] P. L. Larkins and P. Hannaford, Z. Phys. D 27, 313 (1993). http://dx.doi.org/10.1007/BF01437462

[9] E. Peik, G. Hollemann, and H. Walther, Phys. Scr. T59, 403 (1995). http://dx.doi.org/10.1088/0031-8949/1995/T59/055

[10] J. von Zanthier, Th. Becker, M. Eichenseer, A. Yu. Nevsky, Ch. Schwedes, E. Peik, H. Walther, R. Holzwarth, J. Reichert, Th. Udem, T. W. Hänsch, P. V. Pokasov, M. N. Skvortsov, and S. N. Bagayev, Laser Phys. 11 (10), 1117 (2001).

[11] H. Karlsson and U. Litzén, J. Phys. B 34, 4475 (2001). http://dx.doi.org/10.1088/0953-4075/34/22/315

[12] Yu. M. Smirnov, Opt. Spectrosc. 108, 677 (2010). http://dx.doi.org/10.1134/S0030400X10050036

[13] W. F. Meggers, C. H. Corliss, and B. F. Scribner, Tables of Spectral-Line Intensities, Part I - Arranged by Elements, Part II Arranged by Wavelengths, Natl. Bur. Stand. Monograph 145, Nat. Bur. Stand., U.S., 600 pp. (1975).

[14] J. Reader, C. H. Corliss, W. L. Wiese, and G. A. Martin, Wavelengths and Transition Probabilities for Atoms and Atomic Ions, Part. I. Wavelengths, Part II. Transition Probabilities, Nat. Stand. Ref. Data Ser., NSRDS-NBS 68, U.S. Government Printing Office, Washington, D.C., 415 pp. (1980).

[15] K. Wagatsuma, J. Anal. At. Spectrom. 11, 957 (1996). http://dx.doi.org/10.1039/ja9961100957

[16] D. R. Lide, CRC Handbook of Chemistry and Physics, 92nd Edition, Taylor and Francis Group, LLC (2012); Available online at http://www.hbcpnetbase.com/.

[17] G. Norlén, Phys. Scr. 8, 249 (1973). http://dx.doi.org/10.1088/0031-8949/8/6/007

[18] R. C. M. Learner and A. P. Thorne, J. Opt. Soc. Am. B 5, 2045 (1988). http://dx.doi.org/10.1364/JOSAB.5.002045

[19] G. Nave, R. C. M. Learner, A. P. Thorne, and C. J. Harris, J. Opt. Soc. Am. B 8, 2028 (1991). http://dx.doi.org/10.1364/JOSAB.8.002028

[20] G. Nave and C. J. Sansonetti, J. Opt. Soc. Am. B 28, 737 (2011). http://dx.doi.org/10.1364/JOSAB.28.000737

[21] A. E. Kramida, At. Data Nucl. Data Tables 96, 586 (2010). http://dx.doi.org/10.1016/j.adt.2010.05.001

[22] A. E. Kramida and G. Nave, Eur. Phys. J. D 39, 331 (2006). http://dx.doi.org/10.1140/epjd/e2006-00121-4

[23] A. E. Kramida, Comput. Phys. Commun. 182, 419 (2011). http://dx.doi.org/10.1016/j.cpc.2010.09.019

[24] T. R. O'Brian, M. E. Wickliffe, J. E. Lawler, W. Whaling, and J. W. Brault, J. Opt. Soc. Am. B 8, 1185 (1991). http://dx.doi.org/10.1364/JOSAB.8.001185

[25] J. Reader, private communication (2011).

[26] Yu. Ralchenko, A. Kramida, J. Reader, and NIST ASD Team, NIST Atomic Spectra Database (version 4.1), National Institute of Standards and Technology, Gaithersburg, MD; available at: http://physics.nist.gov/asd (2011).

[27] Y. H. Wang, R. Dumke, J. Zhang, T. Liu, A. Stejskal, Y. N. Zhao, Z. H. Lu, L. J. Wang, Th. Becker, and H. Walther, Eur. Phys. J. D 44, 307 (2007). http://dx.doi.org/10.1140/epjd/e2007-00171-0

[28] E. R. Peck and K. Reeder, J. Opt. Soc. Am. 62, 958 (1972). http://dx.doi.org/10.1364/JOSA.62.000958 
[29] C. J. Sansonetti, Computer programs RITZPL and POLAR, private communication (2005).

[30] R. D. Cowan, The Theory of Atomic Structure and Spectra, University of California Press, Berkeley, CA, 731 pp. (1981); A version of the codes adapted for personal computers by A. Kramida is available online at http://das101.isan.troitsk.ru.

[31] D. R. Beck and D. Datta, Phys. Rev. A 44, 758 (1991). http://dx.doi.org/10.1103/PhysRevA.44.758

[32] H. Dahmen and S. Penselin, Z. Phys. 200, 456 (1967). http://dx.doi.org/10.1007/BF01326186

[33] M. W. S. M. Brimicombe, D. N. Stacey, V. Stacey, H. Hühnermann, and N. Menzel, Proc. R. Soc. London, Ser. A 352, 141 (1976). http://dx.doi.org/10.1098/rspa.1976.0168

[34] D. C. Morton, Q. Wu, and G. W. F. Drake, Can. J. Phys. 84, 83 (2006). http://dx.doi.org/10.1139/p06-009

[35] E. Biémont and C. J. Zeippen, At. Data Nucl. Data Tables 72, 101 (1999). http://dx.doi.org/10.1006/adnd.1999.0810

[36] A. Kramida and J.R. Fuhr, NIST Atomic Transition Probability Bibliographic Database (version 9.0), National Institute of Standards and Technology, Gaithersburg, MD (2010). Available at: http://physics.nist.gov/Fvalbib.

[37] Th. Becker, J. von Zanthier, A. Yu. Nevsky, Ch. Schwedes, M. N. Skvortsov, H. Walther, and E. Peik, Phys. Rev. A 63, 051802 (2001). http://dx.doi.org/10.1103/PhysRevA.63.051802

[38] W. Ansbacher, E. H. Pinnington, J. A. Kernahan, and R. N. Gosselin, Can. J. Phys. 64, 1365 (1986). http://dx.doi.org/10.1139/p86-242

[39] L. J. Curtis, R. Matulioniene, D. G. Ellis, and C. Froese Fischer, Phys. Rev. A 62, 052513 (2000). http://dx.doi.org/10.1103/PhysRevA.62.052513

[40] E. Peik, G. Hollemann, and H. Walther, Phys. Rev. A 49, 402 (1994). http://dx.doi.org/10.1103/PhysRevA.49.402

[41] B. Martinez, F. Blanco, and J. Campos, J. Phys. B 29, 2997 (1996). http://dx.doi.org/10.1088/0953-4075/29/14/012

[42] K. B. Blagoev, Ya. F. Verolainen, V. N. Gorshkov, and V. A. Komarovskii, Opt. Spectrosc. 59, 566 (1985).

[43] P. Jönsson and M. Andersson, J. Phys. B 40, 2417 (2007). http://dx.doi.org/10.1088/0953-4075/40/12/016

[44] C. Lavín and I. Martin, J. Quant. Spectrosc. Radiat. Transfer 52, 21 (1994). http://dx.doi.org/10.1016/0022-4073(94)90135-X

[45] T. Andersen and G. Sørensen, Phys. Rev. A 5, 2447 (1972). http://dx.doi.org/10.1103/PhysRevA.5.2447

About the author: Alexander Kramida is a physicist in the Atomic Spectroscopy Group of the Quantum Measurement Division, Physical Measurement Laboratory at the National Institute of Standards and Technology, Gaithersburg, MD. The National Institute of Standards and Technology is an agency of the U.S. Department of Commerce. 